\begin{tabular}{ccr} 
FOLIA & ENTOMOLOGICA & HUNGAR ICA \\
& ROVARTANI KÖZLEMÉNYEK & \\
Volume 80 & 2019 & pp. 89-230 \\
\hline
\end{tabular}

\title{
Checklist of the Curculionoidea of Hungary (Coleoptera)
}

\author{
Attila Podlussán ${ }^{1}$, Valentin SzÉNÁsI ${ }^{2 *}$ \& Ottó Mer KL ${ }^{3}$ \\ ${ }^{1}$ H-1117 Budapest, Bercsényi utca 10, Hungary.E-mail:podlussany.1941@gmail.com \\ ${ }^{2}$ Duna-Ipoly National Park Directorate, H-1021 Budapest, Költö utca 21, Hungary. \\ E-mail:szvalent@gmail.com \\ ${ }^{3}$ Hungarian Natural History Museum, Department of Zoology, \\ H-1088 Budapest,Barossutca 13, Hungary.E-mail:merkl.otto@nhmus.hu
}

\begin{abstract}
The last checklist of the superfamily Curculionoidea of Hungary was published in 1996. Between 1996 and 2019, 68 species were added to the Hungarian fauna. This paper lists 1224 species belonging to 329 genera of 5 families from present-day Hungary. Taxonomical changes and erroneous country records in the literature are corrected. With 9 figures.
\end{abstract}

Key words - catalogue, faunistics, weevils

\section{INTRODUCTION}

The last comprehensive checklist of the superfamily Curculionoidea of Hungary is more than 20 years old (PodLussánY 1996). Since then several papers were published on species new to the fauna of Hungary (e. g. KRÁt KÝ \& Podlussány 2008, MER KL et al. 2010, 2012, Podlussány 2001b, PodlussánY et al. 2016, 2017, SzÉNÁsi 2014, 2016, 2018) or to the science (e. g. BIAŁOOKI 2007, Yuna Kov 2006), as well as substantial catalogues on the Palaearctic Curculionoidea appeared (Löвl \& SMetana 2011, 2013, Alonso-Zarazaga et al. 2017). The latter catalogues contain about 150 errors regarding Hungarian distributions: species are mentioned from Hungary, but in fact they do not occur in the country; or inversely so, Hungary is missing from the list of countries of certain species which do occur there.

The checklist with 1224 species belonging to 329 genera of 5 families presented hereunder is devoted to update the taxonomical and nomenclatural situation of the Hungarian Curculionoidea, to complete it with the species recorded since 1996, and to delete the names erroneously reported from Hungary.

${ }^{*}$ Corresponding author. 
Two enigmatic species, Corimalia maculaticeps (Pic, 1929) and Apsis bungarica (Formánek, 1925), were described from Hungary, but since their description they were never found again. With no access to the types their validity could not be confirmed.

\section{SPECIES DELETED FROM THE HUNGARIAN FAUNAL LIST}

Ninety-seven species listed hereunder are mentioned to occur in Hungary in Löbl \& Smetana $(2011,2013)$ and in Alonso-Zarazaga et al. (2017), but they should be deleted from the Hungarian faunal list. The main reasons of deletion are the following.

\section{Misidentifications.}

2. Species are mentioned from the territory of the Kingdom of Hungary that was much larger than the country after the Treaty of Trianon in 1920. Many species occur in areas now belonging to the surrounding countries, but are unknown from present-day Hungary (most of them live in higher elevations of the Carpathians or in southern areas such as the Banat or Vojvodina).

3. Species are mentioned only with the name of the country or a poorly defined region (e.g. "Hungaria" or "Hungaria meridionalis"); voucher specimens, if exist at all, are without more precise locality.

\section{Lack of voucher specimens.}

5. Voucher specimens exist but their origin is unreliable or unrealistic (e.g. many specimens with label text "Siófok" from the collection of Ferenc Lichtneckert; he lived in and collected around the Transdanubian town Siófok, but collected or obtained specimens from other counties as well; after his death all unlabelled specimens were heedlessly tagged with labels reading "Siófok", no matter where the specimens came from).

6. Taxonomic revisions. After revisions of genera or species groups it turned out that some species previously believed to present in Hungary in fact absent in the country; instead, the specimens from Hungary belong to another species. Examples include Aphytobius sphaerion vs. veronicae (KRÁtKÝ 2015), Minyops carinatus vs. variolosus (OsElla \& Bellò 2010) or Mogulones sublineellus vs. albolineatus (KRÁT KÝ \& SZYPUŁA 2018). 
Brentidae

Ceratapion transsylvanicum (Schilsky, 1906)

Hemitrichapion lethierryi (Desbrochers des Loges, 1870)

Nanodiscus transversus (Aubé, 1850)

Curculionidae

Amicromias intermedius Yunakov, 2005

Amicromias rumelicus (Apfelbeck, 1899)

Anthonomus spilotus L. Redtenbacher, 1847

Aphytobius sphaerion (Boheman, 1845)

Asproparthenis albicans Gyllenhal, 1834

Bagous diglyptus Boheman, 1845

Bangasternus orientalis (Capiomont,1873)

Baris nesapia Faust, 1887

Barynotus hungaricus (Tournier, 1876)

Barynotus moerens (Fabricius, 1792)

Ceutorhynchus laetus Rosenhauer, 1856

Ceutorhynchus liliputanus Apfelbeck, 1908

Ceutorhynchus querceti Gyllenhal, 1813

Ceutorhynchus thlaspi

C. N. F. Brisout de Barneville, 1869

Ceutorhynchus viridanus Gyllenhal, 1837

Chlorophanus dorsiger Faust, 1897

Cionus ungulatus Germar, 1821

Cirrorbynchus crinipes (Miller, 1863)

Cirrorhynchus argenteus (Stierlin, 1861)

Cleopomiarus longirostris (Gyllenhal, 1838)

Cleopomiarus medius (Desbrochers des Loges, 1893)

Coniocleonus hollbergii (Fåhraeus, 1842)

Coniocleonus nebulosus (Linnaeus, 1758)

Cotaster uncipes (Boheman, 1838)

Cycloderes canescens (Rossi, 1792)

Dodecastichus contractus (Stierlin, 1861)

Dodecastichus heydenii (Stierlin, 1861)

Dodecastichus obsoletus (Stierlin, 1861)

Donus cyrtus (Germar, 1821)

Donus rubi Krauss, 1900

Dorytomus edoughensis Desbrochers des Loges, 1875

Gymnetron niloticum Kirsch, 1881

Hypera ononidis (Chevrolat, 1863)

Hypera pastinacae (Rossi, 1790)

Larinus planus (Fabricius, 1792)

Limatogaster pachyscelis (Stierlin, 1861)

Lixus ascanii (Linnaeus, 1767)

Lixus neglectus Fremuth, 1983

Mecinus caucasicus (Reitter, 1907)

Miarus banaticus Reitter, 1907

Miarus campanulae (Linnaeus, 1767)

Miarus ursinus Abeille de Perrin, 1906

Minyops carinatus (Linnaeus, 1767)

Mogulones beckeri (Schultze, 1900)

Mogulones lineatus (Gyllenhal, 1837)
Mogulones sublineellus

(C. N. F. Brisout de Barneville, 1869)

Mylacomorphus globus (Seidlitz, 1868)

Otiorhynchus alpicola Boheman, 1842

Otiorhynchus alpigradus Miller, 1859

Otiorhynchus armatus Boheman, 1842

Otiorhynchus aurifer Boheman, 1842

Otiorhynchus chrysocomus Germar, 1823

Otiorhynchus chrysonus Boheman, 1842

Otiorhynchus corvus Boheman, 1842

Otiorhynchus cymophanus Germar, 1839;

Otiorbynchus dives Germar, 1839

Otiorhynchus edentatus Seidlitz, 1891

Otiorhynchus friulicus (Herbst, 1797)

Otiorbynchus fussianus Csiki, 1901

Otiorhynchus globus Boheman, 1842

Otiorhynchus granicollis Boheman, 1842

Otiorhynchus intercalaris Boheman, 1842

Otiorhynchus krattereri Boheman, 1842

Otiorhynchus kuenburgi Stierlin, 1866

Otiorhynchus lepidopterus (Fabricius, 1794)

Otiorhynchus lithanthracius Boheman, 1834

Otiorhynchus maxillosus Gyllenhal, 1834

Otiorhynchus morio (Fabricius, 1781)

Otiorbynchus necessarius Stierlin, 1861

Otiorhynchus obsidianus Boheman, 1842

Otiorhynchus ormayi Stierlin, 1888

Otiorhynchus peneckianus Smreczyński, 1963

Otiorhynchus petrensis Boheman, 1842

Otiorhynchus polycoccus Gyllenhal, 1842

Otiorhynchus praecellens Stierlin, 1886

Otiorhynchus pupillatus Gyllenhal, 1834

Otiorhynchus remotegranulatus Stierlin, 1891

Otiorhynchus schaumii Stierlin, 1861

Otiorhynchus seductor Stierlin, 1861

Otiorhynchus simplicatus Stierlin, 1861

Otiorhynchus singularis (Linnaeus, 1767)

Otiorhynchus tristis (Scopoli, 1763)

Pityokteines spinidens (Reitter, 1895)

Pityophthorus buyssoni Reitter, 1901

Polydrusus cephalotes Desbrochers des Loges, 1872

Polydrusus pauper (Stierlin, 1890)

Pseudorchestes persimilis (Reitter, 1911)

Sciaphobus rubi (Gyllenhal, 1813)

Smicronyx cyaneus (Gyllenhal, 1935)

Thamiocolus sablbergi (C. R. Sahlberg, 1845)

Thamiocolus sinapis

(Desbrochers des Loges, 1893)

Trachyphloeus rectus C. G. Thomson, 1865

Tropiphorus terricola (Newman, 1838)

Tychius striatulus Gyllenhal, 1835 

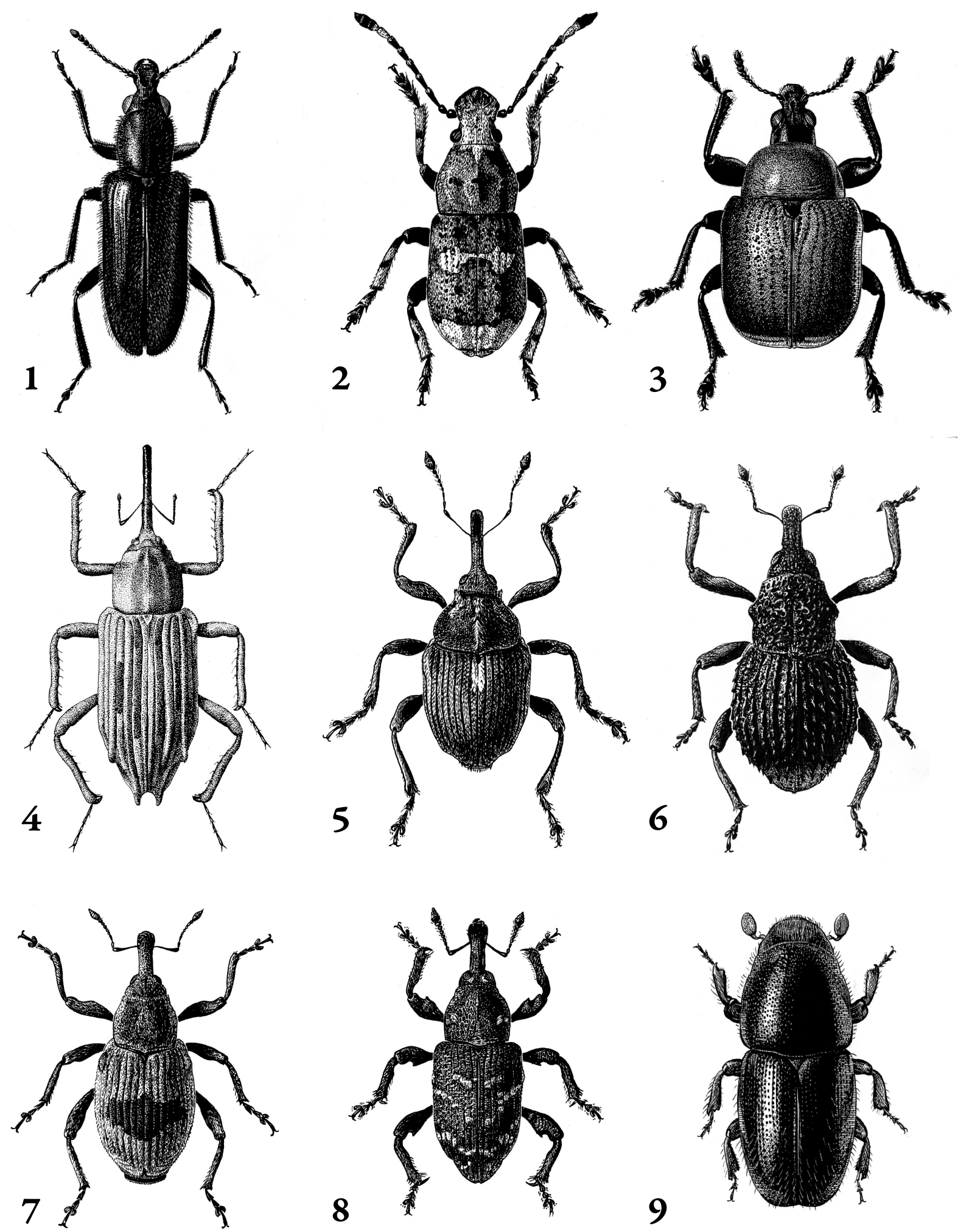

Figs 1-9. 1 = Nemonyx lepturoides (Fabricius, 1801), $2=$ Platystomos albinus (Linnaeus, 1758), $3=$ Attelabus nitens (Scopoli, 1763), 4 = Bagous elegans (Fabricius, 1801), 5 = Glocianus maculaalba (Herbst, 1795), $6=$ Scleropterus serratus (Germar, 1824), 7 = Tapinotus sellatus (Fabricius, 1794), $8=$ Hylobius abietis (Linnaeus, 1758), 9 = Scolytus mali (Bechstein, 1805). Not to scale. Sources: 1, 3 = ENDRöDI (1958); 2 = ENDRÖDI (1961a), 4 = ENDRÖDI (1971), 5-7 = ENDRÖDI (1968); 8 = ENDRÖDI (1963); 9 = ENDRÖDI (1959)

(drawings by Gyula Keve) 


\title{
DATA PRESENTATION
}

The systematic array of the taxa above species-group level follows ALonsoZARAZAGA et al. (2017), but for the sake of simplicity, the subtribes are omitted. Genera, subgenera and species-group names are in alphabetical order. Family names are in REGULAR ALL CAPS, subfamilies are in Bold lowercase, supertribes (in the subfamily Conoderinae) are in SMALL CAPS, tribes are Regular lower case, genera are Bold italics lowercase, subgenera are Regular italics lowercase - all are with centered alignment. The species-group names are in Regular italics lowercase and their entries are with justified alignment.

If a species-group name is mentioned without citing a literature source, the name was included in the checklist of PoduUssánY (1996). When the name is followed by a reference, the species was reported as new to Hungary after 1996, and the reference contains the first record. Synonymy is restricted to names mentioned in the Hungarian literature.

Hungarian name is also provided for every species, with reference(s) if it was already accepted previously; the lack of reference means that the name is introduced for the first time in this paper. Other, less frequently used (or sometimes misleading) Hungarian names found in the literature are also listed and referenced, with no claim to be exhaustive, but focusing mainly on names of agricultural, stored product and timber pests.

\section{THE CHECKLIST}

\author{
NEMONYCHIDAE Bedel, 1882 \\ Cimberidinae Gozis, 1882 \\ Cimberidini Gozis, 1882
}

Cimberis Gozis, 1881

Cimberis attelaboides (Fabricius, 1787) - fekete fenyő-áleszelény (MER KL \& VIG 2009) - Other names: törpe-fenyőormányos, fenyő-áleszelény (GozMÁNY 1979); fenyő-áleszelény (ENDRődI 1958, PODLUSSÁNY 1984) 


\section{Doydirhynchini Pierce, 1916 \\ Doydirbynchus Dejean, 1821}

Doydirhynchus austriacus (Olivier, 1807) - osztrák fenyö-áleszelény (MERKL \& VIG 2009) - Other names: osztrák áleszelény (ENDRődi 1958, Jolsvay et al. 1977, Podlussány 1984)

Nemonychinae Bedel, 1882

Nemonyx L. Redtenbacher, 1845

Nemonyx lepturoides (Fabricius, 1801) (Fig. 1) - szarkaláb-áleszelény (MERKL \& Vig 2009) - Other names: szarkaláb-félormányos (DUDICH 1950, GoZMÁNY 1979); karcsú áleszelény (ENDRÖDI 1958, GoZMÁNY 1979, JolsVAY et al. 1977, KASZA B 1969, PodLUSSÁNy 1984)

ANTHRIBIDAE Billberg, 1820

Anthribinae Billberg, 1820

Allandrini Billberg, 1820

Allandrus LeConte, 1876

Allandrus undulatus (Panzer, 1795) - hullámos orrosbogár (GYöRgY 2006)

\section{Phaeochrotes Pascoe, 1860}

Phaeochrotes pudens Gyllenhal, 1833 (= cinctus Paykull, 1792) - tölgyorrosbogár (GYÖRGY 2006, MERKL et al.2019)

Anthribini Billberg, 1820

Anthribus Geoffroy, 1762

(= Brachytarsus Schönherr, 1823)

Anthribus fasciatus Forster, 1770 - foltsoros pajzstetvész-orrosbogár (GYöRGY 2006) - Other names: foltsoros pajzstetvész (DU DICH 1950, DU DICH \& LoKsa 1969, Jolsvay et al. 1977, Mer KL \& VIG 2009); foltsoros pajzstetvész, foltos pajzstetvész, öves laposorrú ormányos (GozmáNY 1979) 
Anthribus nebulosus Forster, 1770 - ködfoltos pajzstetvész-orrosbogár (GYÖRGY 2006, MER KL \& SZÉNÁsI 2018) - Other names: ködfoltos pajzstetvész (Dudich 1950, Dudich \& Loksa 1969, Gozmány 1979, Jolsvay et al. 1977, Merkl \& Vig 2009, Mer KL et al. 2019, Podlussány 2014, SzÉnÁsi 2012)

Discotenini Lacordaire, 1865

Enedreytes Schönherr, 1839

Enedreytes hilaris Fåhraeus, 1839 - kétdudoros orrosbogár (GYöRGY 2006) Other names: vidor bércze (I. FRIVALDSZKY 1865)

Pseudeuparius Jordan, 1914

Pseudeuparius sepicola (Fabricius, 1792) - hatdudoros orrosbogár (GYöRGY 2006)

Platyrhinini Imhoff, 1856

Platyrhinus Clairville, 1798

Platyrhinus resinosus (Scopoli, 1763) - nagy orrosbogár (DUDICH 1950, Dudich \& LoKsa 1969, Gozmány 1979, György 2006, Jolsvay et al. 1977, MERKL \& Vig 2009, MERKL et al. 2019, PodLusSÁny 2009, SzÉNÁsi et al. 2016)

\section{Ulorbinus Sharp, 1891}

Ulorbinusbilineatus(Germar,1819)-dülledtszemüorrosbogár(Du DICH 1950, GYöRgY 2006, PODLUSSÁNY 2009)

\section{Platystomini Pierce, 1916}

\section{Platystomos D. H. Schneider, 1791}

Platystomos albinus (Linnaeus, 1758) (Fig. 2) - nagybajuszú orrosbogár (Dudich 1950, Gozmány 1979, 1969, György 2006, Jolsvay et al. 1977, Merkl et al. 2019, Podlussány 2009, 2014, SzÉNÁsi et al. 2016) - Other names: fehérsávos pajzstetvész, hókás laposorrú ormányos (GozMÁNY 1979); hosszúcsápú orrosbogár (MER KL \& VIG 2009) 
Trigonorhinini Valentine, 1999

Opanthribus Schilsky, 1907

(= Paramesus Fåhraeus, 1781)

Opanthribus tessellatus (Boheman, 1829) - tömzsi orrosbogár (GYöRGY 2006)

Tropiderini Lacordaire, 1865

Gonotropis LeConte, 1876

Gonotropis gibbosa LeConte, 1876 - domború orrosbogár (GYÖRGY 2006) Other names: jegyhátu ajakár (I. FRIVALDSZKY 1865)

Tropideres Schönherr, 1823

Tropideres albirostris (Schaller, 1783) - fehérfoltos orrosbogár (DUDICH 1950, GozMÁNy 1979, GYörgy 2006, MERKL \& SZÉNÁsi 2018, MERKL et al. 2019)

Zygaenodini Lacordaire, 1865

Dissoleucas Jordan, 1925

Dissoleucas niveirostris (Fabricius, 1798) - fehérfarú orrosbogár (DUDICH 1950, György 2006, MERKL et al. 2019, PODLUSSÁNy 2009, 2014, SZÉNÁsi et al. 2016)

Noxius Jordan, 1936

Noxius curtirostris (Mulsant et Rey, 1861) - rövidorrú orrosbogár (GYÖRGY 2006, MERKL et al. 2019)

Rhaphitropis Reitter, 1916

Rhaphitropis marchica (Herbst, 1797) - szalagos orrosbogár (DUDICH 1950, György 2006, MER KL \& SzÉNÁsi 2018, Mer Kl et al. 2019, PodlusSÁny 2014)

Rhaphitropis oxyacanthae (C. N. F. Brisout de Barneville, 1863) - piroslábú orrosbogár (GYÖRGY 2006) 
Choraginae Kirby, 1819

Araecerini Lacordaire, 1865

Araecerus Schönherr, 1823

Araecerus fasciculatus (DeGeer, 1775) (= coffeae Fabricius, 1801) - kávéorrosbogár (GozMÁNY 1979, GYÖRGY 2006, MER KL \& VIG 2009)

Choragini Kirby, 1819

Choragus Kirby, 1819

Choragus horni Wolfrum, 1930 - gombaevő orrosbogár - Other names: Horn-orrosbogár (GYÖRGY 2006)

Choragus sheppardi Kirby, 1819 - vöröslábú orrosbogár - Other names: Sheppard-orrosbogár (GYöRGY 2006)

Melanopsacus Jordan, 1924

Melanopsacus grenieri (C. N. F. Brisout de Barneville, 1867) (GYörgY \& PODLUSSÁNY 2005) - nagyszemű orrosbogár (GYÖRGY 2006)

\section{Pseudochoragus Petri, 1912}

Pseudochoragus piceus (Schaum, 1845) - parányi orrosbogár (GYöRGY 2006, MERKL \& VIG 2009)

Urodontinae C. G. Thomson, 1859

Bruchela Dejean, 1821

Bruchela cana (Küster, 1848) - déli rezedabogár (GYöRGY 2006)

Bruchela conformis (Gyllenhal, 1833) (KRÁTKÝ \& PODLUSSÁNy 2008) - tar rezedabogár (DUDICH 1950)

Bruchela kaszabi (Strejček, 1973) (= orientalis Strejček, 1982) - keleti rezedabogár (GYÖRgY 2006, MER KL \& SZÉNÁSI 2018)

Bruchela rufipes rufipes (Olivier, 1790) - öszes rezedabogár (DuDICH 1950, Dudich \& LoKsA 1969, György 2006, Jolsvay et al. 1977) 
Bruchela schusteri (Schilsky, 1912) (Poduussány 2001b) - Schusterrezedabogár (GYÖRGY 2006, MER KL \& SZÉNÁSI 2018)

Bruchela suturalis (Fabricius, 1792) - sávos rezedabogár (DUDICH 1950, Dudich \& Loksa 1969, György 2006, Jolsvay et al. 1977, Merkl \& Vig 2009, MERKL et al. 2019, PodLUSSÁNy 2014)

\author{
ATTELABIDAE Billberg, 1820 \\ Attelabinae Billberg, 1820 \\ Apoderini Jekel, 1860
}

Apoderus Olivier, 1807

Apoderus Olivier, 1807

Apoderus (Apoderus) coryli (Linnaeus, 1758) - mogyoró-levélsodró (DuDICH 1950, DUdiCH \& LoKsA 1969, ENDRŐdi 1958, GoZMÁ NY 1979, PODLUSSÁNY 1984, 2010, Reichart in Jermy \& BALÁzs 1990, Tóth 1999) - Other names: piros tevenyakú bogár, tevenyakú mogyoróbogár, mogyoró vastagorrú bogara (GOZMÁNY 1979); mogyoróeszelény (piros tevenyakú bogár, mogyorón élő vastagszájú ormányos) (REICHART in JERMY \& BALÁzs 1990, Tóth 1999); mogyoró-levélpödrő (MER KL \& VIG 2009)

\title{
Compsapoderus Voss, 1927 \\ Compsapoderus Voss, 1927
}

Compsapoderus (Compsapoderus) erythropterus (Gmelin, 1790) - füzikelevélsodró - Other names: szeder-levélsodró (DUDICH 1950); füzike-levélpödrő (MERKL \& VIG 2009)

\author{
Attelabini Billberg, 1820 \\ Attelabus Linnaeus, 1758
}

Attelabus nitens (Scopoli, 1763) (Fig. 3) - tölgy-levélsodró (Dudich 1950, Dudich \& LOKSA 1969, ENDRődi 1958, GoZmánY 1979, MER KL \& Vig 2009, MerkL et al. 2019, PoDlussány 1984, 2014) - Other names: tölgy levélsodró bogara (GOZMÁNY 1979) 


\section{Rhynchitinae Gistel, 1848 \\ Auletini Desbrochers des Loges, 1908}

Auletobius Desbrochers des Loges, 1869

Auletobius sanguisorbae (Schrank, 1798) (= basilaris Gyllenhal, 1839) fekete vérfüeszelény - Other names: töves furulyász (I. FRIVALDSZKY 1865); igénytelen furulyász (I. FRIVALDSZKY 1865); vérfüeszelény (ENDRŐDI 1958, Podlussány 1984, Merkl \& Vig 2009)

Byctiscini Voss, 1923

Byctiscus C. G. Thomson, 1859

Byctiscus betulae (Linnaeus, 1758) - szőlö-levélsodró (DuDich 1950, Dudich \& LoKsa 1969, ENDRődi 1958, Podlussány 1984) - Other names: nyírligeti eszelény (DIvald \& WAGNER 1868); szivaros bogár, szőlő-levélsodró, levélsodró szőlőormányos, szőlőlevél-ormányos, szivarsodró eszelény, trafikos bogár (GOZMÁNY 1979); szivarsodró eszelény (BALÁs \& SÁRINGER 1984); szivarsodróormányos (SURÁ NY I 1946); szőlőlevélsodró eszelény (PÉTER FI 1958); szivarsodró eszelény (szőlőlevélsodró eszelény, szőlőlevélcsavaró bogár, szőlőlevél ormányos, körteeszelény, vincur, szivarsodró ormányos) (REICH ART in JER MY \& BALÁzs 1990); szőlőeszelény(BEzsilla et al. 1953, Gozmány 1979, Jablonowski 1912, Kadocsa 1942, Merkl \& SzénÁsi 2018, Merkl \& Vig 2009, Merkl et al. 2019, Péterfi 1958, Reichart in Jermy \& Balázs 1990, SZÉNÁsi 2012, Tó TH 1999, UBRIZSY \& REICHART 1958); szőlő levélsodró (szivarsodró eszelény) (Tó TH 1999)

Byctiscuspopuli (Linnaeus, 1758) - nyárfa-levélsodró (ENDRőDI 1958, KASZAB 1969, PodLuSSÁ N Y 1984, Tó TH 1999) - Other names: nyárfasodró (DUDICH 1950); nyárfalevélsodró ormányos (REICHART 1953); nyárfalevélsodró (GoZMÁNY 1979, REICHART in JERMY \& BALÁzS 1990); nyáreszelény (BALÁs \& SÁRINGER 1984, MERKL \& Vig 2009, SZÉnÁsi 2012); nyárfaeszelény (Gozmány 1979, MERKL \& SZÉNÁSI 2018, REICHART in JERMy \& BALÁzs 1990)

\section{Deporaini Voss, 1929}

\section{Chonostropheus Prell, 1924}

Chonostropheus tristis (Fabricius, 1794) - juhar-levélsodró (DUDICH 1950, Dudich \& LoKsa 1969, Endrödi 1958, Gozmány 1979, Podlussány 1984, Tóth 1999) - Other names: juhareszelény (MER KL \& VIG 2009, PodLUSSÁNY 2014) 
Deporaus Samouelle, 1819

Caenorbinus C. G. Thomson, 1859

Deporaus (Caenorbinus) megacephalus (Germar, 1823) (= mannerheimii Hummel, 1823) - kékes levélsodró

\title{
Deporaus Samouelle, 1819
}

Deporaus (Deporaus) betulae (Linnaeus, 1758) - nyírfa-levélsodró (DUDICH 1950, DUDICH \& LOKSA 1969, ENDRőDI 1958, GOZMÁNY 1979, MER KL etal.2019, PoDLUSSÁNY 1984,2010, TóTH 1999) - Other names: nyírfalevélsodró ormányos, nyárfa ugróormányos bogara (GOZMÁNY 1979); nyíreszelény (MERKL \& VIG 2009)

\author{
Rhynchitini Gistel, 1848 \\ Involvulus Schrank, 1798 \\ Involvulus Schrank, 1798
}

Involvulus (Involvulus) cupreus (Linnaeus, 1758) - rezes szilvaeszelény Other names: szilvaeszelény (BALÁs \& SÁRINGER 1984, Dudich 1950, DUdich \& LoKsa 1969, ENDRŐdi 1958, Jolsvay et al. 1977, Merkl \& Vig 2009, Tóth 1999, UBRIZsY \& REICHART 1958); kökényeszelény (BALÁs 1966); szilvaormányos, kökényeszelény, szilvaeszelény (GozMÁNY 1979); szilvaeszelény (kökényeszelény) (REICHART in JERMY \& BALÁzs 1990)

\section{Teretriorhynchites Voss, 1938}

Involvulus (Teretriorhynchites) icosandriae icosandriae (Scopoli, 1763) (= caeruleus DeGeer, 1775) - hajtástörő eszelény (BALÁs \& SÁRINGER 1984, DUdiCH 1950, Dudich \& LOKSA 1969, ENDRŐdi 1968, GOZMÁNY 1979, JolsVAY et al. 1977, MERKL \& SzÉNÁsi 2018, MERKL \& Vig 2009, PodLusSÁNy 1984, 2009, ReichaRT in JeRmy \& BALÁzs 1990, SzÉNÁsi et al. 2016, Ubrizsy \& REICHART 1958) - Other names: hajtásfúró ormányos, hajtásfúró eszelény (GOZM ÁNY 1979); hajtásfúró eszelény (JABLONOWSKI 1912); hajtásfúró eszelény, égkék levélszúró eszelény (REICHART in JERMY \& BALÁzs 1990)

Involvulus (Teretriorhynchites) pubescens (Fabricius, 1775) - szörös eszelény (ENDRöDi 1968, MERKL \& SZÉNÁsi 2018, MERKL \& Vig 2009) 
Lasiorhynchites Jekel, 1860

Coccygorrhynchites Prell, 1926

Lasiorhynchites (Coccygorrhynchites) sericeus (Herbst, 1797) - kék kakukkeszelény - Other names: selymes eszelény (I. FRIVALDSZKY 1865, DUDICH 1950, GOZMÁNY 1979); kakukkeszelény (ENDRŐDI 1958, GOZMÁNY 1979, JolsVAY et al. 1977, Kaszab 1969, Merkl \& Vig 2009, Podlussány 1984)

Lasiorhynchites Jekel, 1860

Lasiorhynchites (Lasiorbynchites) cavifrons (Gyllenhal, 1833) - erdei eszelény (ENDRödi 1958, Gozmány 1979, Jolsvay et al. 1977, Merkl \& Vig 2009, PODLUSSÁNY 1984, 2014)

Nelasiorhynchites Legalov, 2003

Lasiorhynchites (Nelasiorhynchites) comatus (Gyllenhal, 1833) (= olivaceus Gyllenhal, 1833) - közönséges tölgyeszelény (MER KL et al. 2019) - Other names: tölgyeszelény (Dudich 1950, EndRöDi 1958, Jolsvay et al. 1977, Merk \& \& VIG 2009, PODLUSSÁNY 1984, 2009)

Lasiorhynchites (Nelasiorhynchites) praeustus (Boheman, 1845) - vörös eszelény (ENDRődi 1958, JolsvaY et al. 1977, MERKL \& Vig 2009, PodLusSÁny 1984)

Stenorhynchites Voss, 1932

Lasiorhynchites (Stenorbynchites) caeruleocephalus (Schaller, 1783) - kékfejü eszelény (ENDRöDi 1958, JolsVAY et al. 1977, MERKL \& Vig 2009, PODLussánY 1984)

Mecorbis Billberg, 1820

Mecorhis Billberg, 1820

Mecorhis (Mecorhis) ungarica (Herbst, 1783) - magyar eszelény (DuDICH 1950, Dudich \& LoKsa 1969, EndRöDi 1958, Gozmány 1979, Jolsvay et al. 1977, PODLUSSÁNY 1984) - Other names: magyarhoni eszelény (I. FRIVALDSZKY 1865) 
Pseudomechoris Legalov, 2003

Mecorhis (Pseudomechoris) aethiops (Bach, 1854) - fekete eszelény - Other names: szerecseneszelény (DUDICH 1950, ENDRÖDI 1958, JolSVAY et al. 1977, PodLUSSÁNY 1984)

Neocoenorrbinus Voss, 1952

Neocoenorbinidius Legalov, 2003

Neocoenorrbinus (Neocoenorbinidius) interpunctatus (Stephens, 1831) levéléreszelény (MERKL et al. 2019, REICHART in JERMy \& BALÁzs 1990, Tót H 1999) - Other names: földieper levélfúró eszelény, levélfúró eszelény, levélszúró ormányos, levélszúró eszelény, apróka levélszúró eszelény (REICH ART in JERMY \& BALÁZS 1990)

Neocoenorrbinus (Neocoenorbinidius) pauxillus (Germar, 1824) - bordafúró eszelény (ENDRődi 1958, Jolsvay et al. 1977, KASZA B 1969, MER KL \& SzÉNÁsi 2018, Merkl \& Vig 2009, Merkl et al. 2019, Podlussány 1984, 2009, 2014, REICHART in JERMY \& BALÁzs 1990) - Other names: levélbordafúró eszelény (KAdocsa 1942, PÉTERFi 1958); levélnyélfúró eszelény (SURÁNYi 1946); galagonyaeszelény (Bezsilla et al. 1953, Dudich 1950, PÉterfi 1958); levélfúró eszelény, galagonya-eszelény (JoLSVAY et al. 1977); levélfúró eszelény (levélbordafúró eszelény, pontokkal megkülönböztetett levélszúró eszelény) (REICHART in JERMY \& BALÁzs 1990); levélfúró eszelény (BALÁs \& SÁRINGER 1984, Harmos et al. 2002, Tó Th 1999, Ubrizsy \& REICHART 1958)

\section{Neocoenorrhinus Voss, 1952}

Neocoenorrbinus (Neocoenorrbinus) germanicus (Herbst, 1797) - közönséges szamócaeszelény (MERKL et al. 2019) - Other names: német eszelény (DUDICH 1950); szamócaeszelény (BALÁs \& SÁRINGER 1984, ENDRődi 1958, JolsVAY et al. 1977, Kaszab 1969, Podlussány 1984, 2009, 2010, SzÉnÁsi et al. 2016, Merkl \& Vig 2009, Merkl \& SzénÁsi 2018, Tóth 1999, Ubrizsy \& REICHART 1958); szamócaeszelény (eperormányos, németeszelény, német levélszúró eszelény) (REICHART in JERMY \& BALÁzs 1990); sötétkék eszelény, szamócaeszelény, német eszelény (Tó TH 1999) 
Schoenitemnus Legalov, 2003

Neocoenorrbinus (Schoenitemnus) minutus (Herbst, 1797) (= aeneovirens Marsham, 1802) - bronzos eszelény (ENDRöDI 1958, JolsvaY et al. 1977, MER KL \& VIg 2009, PodlussáNy 1984) - Other names: szamócaeszelény (DUdich 1950)

Rhynchites D. H. Schneider, 1791

Epirhynchites Voss, 1969

Rhynchites (Epirhynchites) auratus (Scopoli, 1763) ) - aranyos eszelény (BALÁs \& SÁRINGER 1984,DUDICH 1950,DUDICH \&LOKSA 1969, ENDRŐDI 1958, Jolsvay et al. 1977, Mer KL \& SzÉNÁsi 2018, MER KL \& Vig 2009, MER KL et al. 2019, Podlussány 1984, Reichart in JeRmy \& BALÁZs 1990, SzÉnÁsi 2012, SZÉNÁsi et al. 2016) - Other names: meggyeszelény (JENSER 1969, PÉT ERFI 1958, UBRIZSY \& REICHART 1958,); szilvaeszelény (JABLONOWSKI 1912); szilvaormányos, meggyeszelény, almaeszelény, kökényeszelény (GoZMÁNY 1979); kökényeszelény (KA DOCSA 1942); almaeszelény (SU RÁ NY I 1946); meggyeszelény, szilvaeszelény, aranyos levélszúró-eszelény, kökény eszelény, alma eszelény (REICHART in JERMY \& BALÁzS 1990)

Rhynchites (Epirhynchites) giganteus Schönherr, $1832 \quad(=$ giganteus Krynicki, 1832) - fémes körteeszelény (MERKL \& SzÉNÁsI 2018) - Other names: körteeszelény (BALÁs \& SÁRINGER 1984, ENDRŐDI 1958, JOLSVAY et al. 1977, Kaszab 1969, Podlussány 1984, Merkl \& Vig 2009, Tóth 1999); körteeszelény (óriás eszelény) (REICHART in JERMY \& BALÁzs 1990); körteormányos, körteeszelény (GozMÁNY 1979)

\section{Rhynchites D. H. Schneider, 1791}

Rhynchites (Rhynchites) bacchus (Linnaeus, 1758) - pompás almaeszelény (Dudich 1950, Gozmány 1979, Péterfi 1958, Reichart in Jermy \& BALÁzs 1990) - Other names: ilonkabogár (SAJó 1894); bíborszínű almafúró (JABLONOWSKI 1900); bíborszínű almafúró bogár, almaeszelény, bíborszínü eszelény, almaormányos (GozMÁNY 1979); almaeszelény (ENDRődi 1958, Kasza B 1969, 2009, Merkl \& Vig 2009, Merkl et al. 2019, Podlussány 1984, UBRIZSY \& REICHART 1958); bíborszínü eszelény (almaeszelény, bíborszínü almafúró bogár, almafúró, levélszúró bachus-eszelény) (REICHART in JERMY \& BALÁzs 1990); bíborszínű eszelény (BALÁs \& SÁRINGER 1984, TóTH 1999)

Rhynchites (Rhynchites) slovenicus Purkyně, 1954 (SzÉNÁsi 2014) barázdásorrú-almaeszelény 
Tatianaerhynchites Legalov, 2002

Tatianaerhynchitesaequatus(Linnaeus, 1767) - piros kökényeszelény-Other names: kökényeszelény (ENDRÖDI 1958, DUDICH 1950, DUDICH \& LOKSA 1969, 2009, 2014, MERKL \& SzÉNÁsi 2018, Merkl \& Vig 2009, Merkl et al. 2019, Podlussány 1984, SzÉNÁsi 2012, SzÉNÁsi et al. 2016); kökényeszelény (pirosszárnyúeszelény)(Jolsvayetal.1977); pirosszárnyúeszelény(PÉT ER FI 1958, UBRIZSY \& REICHART 1958); pirosszárnyú eszelény (veresszárnyú eszelény, kökényeszelény, bíborvörös levélszúró eszelény) (REICHART in JER MY \& BALÁzs 1990, TóTH 1999); veresszárnyú eszelény (BALÁs \& SÁRINGER 1984)

\section{Temnocerus Thunberg, 1815}

Temnocerus coeruleus (Fabricius, 1798) (= tomentosus Gyllenhal, 1839) fogacskás törpeeszelény (MERKL \& SZÉNÁsI 2018) - Other names: simaszőrü eszelény (ENDRődi 1958, PoDLUSSÁNY 1984, SZÉNÁSI 2012)

Temnocerus longiceps (C. G. Thomson, 1888) - szélesfejü törpeeszelény

Temnocerus nanus (Paykull, 1792) - karcsú törpeeszelény - Other names: törpeeszelény (ENDRöDI 1958, JolsvaY et al. 1977, PODLUSSÁNY 1984)

BRENTIDAE Bilberg, 1820

Apioninae Schönherr, 1823

Apionini Schönherr, 1823

Acentrotypus Alonso-Zarazaga, 1990

Acentrotypus brunnipes (Boheman, 1839) - penészvirág-cickányormányos

Aizobius Alonso-Zarazaga, 1990

Aizobius sedi (Germar, 1818) - varjúháj-cickányormányos (SZÉNÁsı 2012)

Alocentron Schilsky, 1901

Alocentron Schilsky, 1901

Alocentron (Alocentron) curvirostre (Gyllenhal, 1833) - mályvaszárcickányormányos (BALÁs \& SÁRINGER 1984, GozmánY 1979, KASZAB 1969, MERKL et al. 2019, SZÉNÁsi 2012) - Other names: hajtottcsőrü hüvelyész 
(I. FRIVALDSZKY 1865); mályvarózsa cickánybogár (DUDICH 1950, GozMÁNY 1979); mályva-cickányormányos (mályvarózsa cickánybogár) (DESEŐ in JER MY \& BALÁZS 1990)

$$
\begin{gathered}
\text { Apion Herbst, } 1797 \\
\text { (= Erythrapion Schilsky, 1906) }
\end{gathered}
$$

Apion cruentatum Walton, 1844 - vérpiros cickányormányos (MERKL 2018, PODLUSSÁNY 2009)

Apion frumentarium (Linnaeus, 1758) (= miniatum Germar, 1833) - vörös cickányormányos (MER KL \& Vig 2009, PodLuSSÁNY 2009, 2014, SZÉNÁsi 2012, SZÉNÁsietal.2016) - Other names: vöröscickánybogár(DUDICH 1950,DUDICH \& LoKsA 1969, GozmánY 1979)

Apion haematodes haematodes Kirby, 1808 - madársóska-cickányormányos (Dudich 1950, Gozmány 1979, Harmos et al. 2002, Merkl 2018, Merkl \& SZÉNÁsI 2018)

Apion rubens Walton, 1837 - pirosló cickányormányos

Apion rubiginosum Grill, 1893 - sóskaszár-cickányormányos (PoDLUSsánY 2009)

Aspidapion Schilsky, 1901

Aspidapion Schilsky, 1901

Aspidapion (Aspidapion) radiolus (Marsham, 1802) - ziliz-cickányormányos (Dudich 1950, Dudich \& LoksA 1969, SzÉNÁsi 2012) - Other names: zilizcickánybogár (GozMÁNY 1979)

Aspidapion (Aspidapion) validum (Germar, 1817) - magaspajzsocskájú cickányormányos (MER KL \& SZÉNÁsi 2018, PODLUSSÁNy 2014, SZÉNÁsi 2012)

Koestlinia Alonso-Zarazaga, 1990

Aspidapion (Koestlinia) aeneum (Fabricius, 1775) - mályvarontó cickányormányos - Other names: fényes cickányormányos (PodLussánY 2014, SZÉNÁSI 2012); mályvaszúró cickánybogár (GOZMÁNY 1979)

Betulapion Ehret, 1994

Betulapionsimile(Kirby,1811)-nyírfa-cickányormányos(MERKLetal.2019)Other names: nyírmag-cickánybogár (GoZMÁNY 1979) 


\section{Catapion Schilsky, 1906}

Catapionjaffense (Desbrochers des Loges, 1895) (= ononiphagum Schatzmayr, 1920) - görbeorrú cickányormányos (MERKL \& SzÉNÁSi 2018, MERKL et al. 2019, Podlussány 2009) ormányos

Catapion meieri (Desbrochers des Loges, 1901) - keskenyorrú cickány-

Catapion pubescens (Kirby, 1811) - széles cickányormányos (MERKL \& SZÉNÁsi 2018, PodlusSÁNy 2009, 2014, SZÉNÁsi 2012)

Catapion seniculus (Kirby, 1808) - lóhereszár-cickányormányos (DEsEÖ in Jermy \& BAlázs 1990, GoZmány 1979, Merkl \& SzÉnÁsi 2018, MerkL et al. 2019, Podlussány 2009, 2010, 2014, SzÉnÁsi 2012) - Other names: szárrontó lóhereormányos (DUDICH 1950); lóhereszár-cickánybogár (UBRIZSY \& REICHART 1958, KASZAB 1969); vöröshereszár-cickányormányos (feketelábú cickánybogár, szárrontó lóhereormányos, vöröshereszár-cickánybogár) (DEsEö in JERMY \& BALÁZs 1990); vöröshereszár-cickányormányos (HARMOs et al. 2002); vöröshereszárny-cickánybogár, szárrontó lóhereormányos, lóhereszárcickánybogár,vöröshere-szárcickánybogár (GOZMÁ NY 1979)

Ceratapion Schilsky, 1901 Acanephodus Alonso-Zarazaga, 1990

Ceratapion (Acanephodus) onopordi onopordi (Kirby, 1808) - szamárbogáncscickányormányos (HARMOS et al. 2002, MERKL 2018, MER KL \& SZÉNÁsi 2018, MER KL et al. 2019, PodLussány 2009, 2010, 2014, SzÉNÁsi 2012, SZÉNÁsi et al. 2016) - Other names: szamárbógáncs cickánybogár (DUDICH 1950)

\section{Angustapion Wanat, 1995}

Ceratapion (Angustapion) austriacum (Wagner, 1904) - osztrák cickányormányos (SZÉNÁsı 2012, SZÉNÁsI et al. 2016)

Ceratapion (Angustapion) cylindricolle (Gyllenhal, 1839) - vasvirágcickányormányos (MER KL \& SZÉNÁsI 2018)

Ceratapion Schilsky, 1901

Ceratapion (Ceratapion) armatum (Gerstäcker, 1854) - imolacickányormányos 
Ceratapion (Ceratapion) carduorum (Kirby, 1808) - bogáncscickányormányos (PoDlussáNY 2009) - Other names: bógáncscickánybogár (DUDICH 1950); articsóka-cickánybogár (GozmÁNY 1979)

Ceratapion (Ceratapion) gibbirostre (Gyllenhal, 1813) - agyaras cickányormányos (MERKL \& SzÉNÁsi 2018, MERkl et al. 2019, PodlusSÁnY 2009, SZÉNÁsi 2012, SZÉNÁsi et al. 2016)

\section{Clementiellus Alonso-Zarazaga, 1990}

Ceratapion (Clementiellus) orientale (Gerstäcker, 1854) - keleti cickányormányos

Echinostroma Alonso-Zarazaga, 1990

Ceratapion (Echinostroma) basicorne(Illiger, 1807)(=alliariaeHerbst, 1797)sarlóslábú cickányormányos

Ceratapion (Echinostroma) penetrans penetrans (Germar, 1817) - lapátlábú cickányormányos (MER KL \& SZÉNÁSI 2018, PodLUSSÁNY 2009, 2010, SZÉNÁSI 2012, SzÉNÁsI et al. 2016)

Cyanapion Bokor, 1923

Bothryorrhynchapion Bokor, 1923

Cyanapion (Bothryorrhynchapion) afer (Gyllenhal, 1833) - pontozottfejü cickányormányos - Other names: kormos cickányormányos (PODLUSSÁNY 2009); hímjehagyott cickányormányos (PodLussánY 2010)

Cyanapion (Bothryorrhynchapion) gyllenhalii (Kirby, 1808) - laposszemü cickányormányos

Cyanapion (Bothryorrhynchapion) platalea (Germar, 1817) - laposorrú cickányormányos (MER KL et al.2019) - Other names: szerecsen-cickányormányos (SZÉNÁsI 2012)

\section{Cyanapion Bokor, 1923}

Cyanapion (Cyanapion) alcyoneum (Germar, 1817) - fémeskék cickányormányos

Cyanapion (Cyanapion) columbinum (Germar, 1817) - lednek-cickányormányos (Merkl et al. 2019, PodLuSSÁNY 2009, 2014, SZÉNÁsi 2012, SzÉNÁsi et al. 2016) Other names: lednekcickánybogár (DuDICH 1950)

Cyanapion (Cyanapion) spencii (Kirby, 1808) - barázdástorú cickányormányos 


\section{Diplapion Reitter, 1916}

Diplapion confluens (Kirby, 1808) - vésetthomlokú cickányormányos (MERKL 2018, MERKL \& SZÉNÁsi 2018, SzÉNÁsi et al. 2016)

Diplapion detritum (Mulsant et Rey, 1858) - kamilla-cickányormányos (Podlussány 2010, SZÉNÁsi 2012, SZÉNÁsi et al. 2016)

Diplapion stolidum (Germar, 1817) - lékeltfejü cickányormányos (MER KL \& SZÉNÁsi 2018, MER KL et al. 2019, PodLussÁNy 2009, 2010, SZÉNÁsi 2012)

\section{Eutrichapion Reitter, 1916}

Cnemapion Bokor, 1923

Eutrichapion (Cnemapion) gribodoi (Desbrochers des Loges, 1896) kecskeruta-cickányormányos - Other-names: Gribodo-cickányormányos (SZÉNÁsI et al. 2016)

Eutrichapion (Cnemapion) vorax (Herbst, 1797) - csavartlábú cickányormányos - Other names: magrontó cickánybogár (GozMÁNY 1979)

Eutrichapion Reitter, 1916 (= Pseudotrichapion Bokor, 1923)

Eutrichapion (Eutrichapion) ervi (Kirby, 1808) - szempillás cickányormányos (MERKL \& SZÉNÁsi 2018, MERKL et al. 2019) - Other names: szempillás cickánybogár (DUDICH 1950); pillás cickányormányos (PoDLUSSÁNY 2009, 2014)

Eutrichapion (Eutrichapion) viciae (Paykull, 1800) - bükkönycickányormányos (DESEŐ in JERMY \& BALÁzs 1990, HARMOS et al. 2002, MER KL \& SZÉNÁsi 2018, MER KL et al. 2019, PODLuSSÁNy 2014, SZÉNÁsi 2012) Other names: bükköny-cickánybogár (DESEÖ in JERMY \& BALÁzs 1990, DUdich 1950, Jolsvay et al. 1977)

\section{Phalacrolobus Alonso-Zarazaga, 1990}

Eutrichapion (Phalacrolobus) melancholicum (Wencker, 1864) - feketés cickányormányos - Other names: komor cickányormányos (SzÉNÁsi et al. 2016)

\section{Psilocalymma Alonso-Zarazaga, 1990}

Eutrichapion (Psilocalymma) facetum (Gyllenhal, 1839) - sötét cickányormányos

Eutrichapion (Psilocalymma) punctiger (Paykull, 1792) - bükkönyrontó cickányormányos(MER KL \& SZÉNÁSI2018, MER KLetal.2019,PODLUSSÁNY2009, 2014, SZÉNÁsi 2012, SZÉNÁsi et al. 2016) - Other names: bükkönyszomorító cickányormányos (PoDLUSSÁNY 2010) 


\section{Exapion Bedel, 1887}

Exapion compactum compactum (Desbrochers des Loges, 1888) - selymes cickányormányos

Exapion corniculatum (Germar, 1817) (= hungaricum Desbrochers des Loges, 1894) - fürtöszanót-cickányormányos - Other names: zanót-cickányormányos (MERKL \& SZÉNÁSI 2018, SZÉNÁSI 2012)

Exapion difficile (Herbst, 1797) - festőrekettye-cickányormányos - Other names: rekettyecickánybogár (DUDICH 1950); rekettye-cickányormányos (MER KL \& SZÉNÁSI 2018, MERKL et al. 2019, SZÉNÁSI 2012)

Exapion elongatulum (Desbrochers des Loges, 1891) - lapított rekettyecickányormányos

Exapion formaneki (Wagner, 1929) - piroslábú rekettye-cickányormányos Other names: Formánek-cickányormányos (MER KL \& SzÉNÁsI 2018)

Exapionfuscirostrefuscirostre(Fabricius, 1775)-fehérsávoscickányormányosOther names: seprőzanót-cickánybogár (GoZMÁNY 1979)

\section{Helianthemapion Wagner, 1930}

Helianthemapion aciculare (Germar, 1817) - napvirág-cickányormányos Helianthemapion velatum (Gerstäcker, 1854) - ércszínủ cickányormányos

Hemitrichapion Voss, 1959

Dimesomyops Alonso-Zarazaga, 1990

Hemitrichapion (Dimesomyops) pavidum (Germar, 1817) - koronafürtcickányormányos (MER KL \& SzÉNÁsi 2018, MERKL et al. 2019, PoDlusSÁNy 2009)

Tinocyba Alonso-Zarazaga, 1990

Hemitrichapion (Tinocyba) reflexum (Gyllenhal, 1833) - fényestorú baltacim-cickányormányos - Other names: baltacim-cickányormányos, baltacim-cickánybogár (DESEŐ in JERMY \& BALÁZs 1990)

Hemitrichapion (Tinocyba)waltoni(Stephens, 1839)(=curtisiStephens, 1831)ércfényü baltacim-cickányormányos 


\section{Holotrichapion Györffy, 1956}

Apions Alonso-Zarazaga, 1990

Holotrichapion (Apions) pisi (Fabricius, 1801) - borsó-cickányormányos (Harmos et al. 2002, Podlussány 2009, 2014, SzÉNÁsi 2012) - Other names: gyepübükköny cickánybogár (DUDICH 1950, GozMÁNY 1979); lucernarügycickánybogár (GOZMÁNY 1979, KASZA B 1969); borsó-cickányormányos (fekete cickánybogár, gyepübükköny-cickánybogár, borsófúró cziczkányocska) (DESEÖ in JERMY \& BALÁzS 1990); lucernarügy-cickányormányos (MERKL 2018, MER KL \& SZÉNÁSI 2018, MER KL et al. 2019, SZÉNÁsi et al. 2016)

Holotrichapion (Apions) pullum (Gyllenhal, 1833)(=aestimatum Faust, 1891)lucernarügy-cickányormányos (DESEŐ in JERMY \& BALÁzs 1990) - Other names: lucerna-cickányormányos, lucerna-cickánybogár (DESEÖ in JERMY \& BALÁZS 1990, SZÉNÁsI 2012); lucernaápion (MANNINGER 1960); lucerna-cickánybogár, lucernaápion (GOZMÁNY 1979); lucerna-cickánybogár (KASZA B 1969); lucernacickányormányos (MER KL et al. 2019); lucerna-cickánybogár (DESEÖ in JER MY \& BALÁZS 1990)

\section{Holotrichapion Györffy, 1956}

Holotrichapion (Holotrichapion) ononis (Kirby, 1808) - szakállas cickányormányos (SZÉNÁsı 2012, SzÉNÁsı et al.2016) - Other names: iglicecickánybogár (Dudich 1950)

\section{Legaricapion Ehret, 1990}

Holotrichapion (Legaricapion) aethiops (Herbst, 1797) - keskenytorú cickányormányos

Holotrichapion (Legaricapion) gracilicolle (Gyllenhal, 1839) - kékhátú cickányormányos

\section{Ischnopterapion Bokor, 1923}

Chlorapion Györffy, 1956

Ischnopterapion (Chlorapion) virens (Herbst, 1797) - lóheregyökércickányormányos(MER KL \& SzÉNÁsı2018, MER KLetal.2019, PoDluss ÁNY2009, 2010, SzÉNÁsi 2012) - Other names: zöld lóhereormányos (DUDICH 1950); lóheregyökér-cickánybogár (UBRIZsY \& REICHART 1958); zöld lóhereormányos (lóheregyőkér-cickánybogár) (Jolsvay et al. 1977, KAsZAB 1969); vörösheregyökér-cickánybogár, zöldlóhereormányos, lóheregyökér-cickánybogár, 
vöröshere-rügycickánybogár (GozMÁNY 1979); vörösheregyökér cickányormányos (MANNINGER 1960); vöröshererügy-cickányormányos (zöld lóhere-cickányormányos, zöld lóhere-ormányos, vöröshererügy-cickánybogár, lóheregyökér-cickánybogár, lóheregyökér-ápion) (DESEÖ in JERMY \& BALÁZS 1990)

\section{Ischnopterapion Bokor, 1923}

Ischnopterapion(Ischnopterapion) aeneomicansaeneomicans(Wencker, 1864)erdőszéli cickányormányos

Ischnopterapion (Ischnopterapion) fallens (Marseul, 1888) - alföldi cickányormányos (MERKL \& SZÉNÁsI 2018)

Ischnopterapion (Ischnopterapion) loti (Kirby, 1808) - szarvaskerepcickányormányos - Other names: kerep-cickányormányos (MERKL \& SZÉNÁsI 2018, MER KL et al. 2019, PodLussánY 2009, 2010, 2014, SZÉNÁsi 2012, SZÉNÁsI et al. 2016); kerepcickánybogár (DUDICH 1950, DUDICH \& LoKSA 1969)

Ischnopterapion (Ischnopterapion) modestum (Germar, 1817) - nagy kerepcickányormányos

Ixapion Roudier et Tempère, 1973

= Ixias Sainte Claire-Deville, 1924)

Ixapionvariegatum(Wencker, 1864)-fagyöngy-cickányormányos(MER KL\& VIG 2009)

\section{Kalcapion Schilsky, 1906}

Kalcapion pallipes (Kirby, 1808) - szélfü-cickányormányos (PoDLUSSÁ NY 2014) ormányos

Kalcapion semivittatum semivittatum (Gyllenhal, 1833) - foltos cickány-

Loborhynchapion Györffy, 1956

Loborhynchapionamethystinum(Miller, 1857)-csüdfüfúrócickányormányos

Malvapion A. Hoffmann, 1958

Malvapion malvae (Fabricius, 1775) - mályva-cickányormányos - Other names: mályva-cickánybogár (GozMÁNY 1979) 


\section{Melanapion Wagner, 1930 \\ Melanapion Wagner, 1930}

Melanapion (Melanapion) minimum (Herbst, 1797) - füz-cickányormányos (MERKL \& SZÉNÁSI 2018)

\section{Mesotrichapion Györffy, 1956}

Mesotrichapion punctirostre (Gyllenhal, 1839) - pontosorrú cickányormányos (MERKL \& SZÉNÁSI 2018, SZÉNÁSI 2012)

\section{Omphalapion Schilsky, 1901}

Omphalapion dispar (Germar, 1817) - pipitér-cickányormányos

Omphalapion hookerorum (Kirby, 1808) (= hookeri Kirby, 1808) (PodlussánY 2001b) - ebszékfü-cickányormányos - Other names: Hookercickányormányos (PoDlussánY 2010, SZÉNÁsI 2012)

Omphalapion laevigatum (Paykull, 1792) (= sorbi Fabricius, 1792) - kamillacickányormányos - Other names: székfü-cickányormányos (DUDICH 1950)

Omphalapion pseudodispar Wanat, 1995 (Podlussány 2001b) - déli cickányormányos

\section{Oryxolaemus Alonso-Zarazaga, 1990}

Oryxolaemus flavifemoratus (Herbst, 1797) - gömbded cickányormányos Other names: rozsdáslábú cickánybogár (GoZMÁNY 1979)

\section{Oxystoma Duméril, 1805}

Oxystoma cerdo (Gerstäcker, 1854) - egérszürke cickányormányos

Oxystoma craccae (Linnaeus, 1767) - kaszanyügbükköny-cickányormányos (Merkl et al. 2019, Podlussány 2009, 2010, 2014) - Other names: kaszanyüg cickánybogár (DUDICH 1950, GoZMÁNY 1979); kaszanyüg-cickányormányos (PodlussánY 2010, SZÉNÁsi 2012, SZÉNÁsi et al. 2016)

Oxystoma dimidiatum (Desbrochers des Loges, 1897) - szürkéskék cickányormányos (MERKL et al. 2019, PODLUSSÁNY 2014)

Oxystoma ochropus (Germar, 1818) - gyepübükköny-cickányormányos Other names: hatalmas cickányormányos (MERKL et al.2019, SzÉNÁsi et al.2016)

Oxystoma opeticum (Bach, 1854) - tavaszilednek-cickányormányos 
Oxystoma pomonae (Fabricius, 1798) - lomblakó cickányormányos (MER KL et al. 2019, PodlussánY 2009) - Other names: lomblakó cickánybogár (DUDICH 1950); gyümölcsfa-cickánybogár, lomblakó cickánybogár, gyümölcsfák cickányorrú bogara (GoZMÁNY 1979)

Oxystoma subulatum (Kirby, 1808) - törösorrú cickányormányos (MERKL et al.2019, PoDlussÁNY 2009, 2010) - Other names: szarvaskerep-cickánybogár (GOZMÁNY 1979)

Perapion Wagner, 1907

Eroosapion Ehret, 1994

Perapion (Eroosapion) lemoroi (C. N. F. Brisout de Barneville, 1880) madárkeserüfü-cickányormányos

Perapion Wagner, 1907

Perapion (Perapion) affine (Kirby, 1808) - kék cickányormányos (MER KL \& SZÉNÁsI 2018, SZÉNÁsI 2012)

Perapion (Perapion) connexum (Schilsky, 1902) (Wanat et al. 2012) hajlottorrú cickányormányos

Perapion (Perapion) curtirostre (Germar, 1817) - kurtaorrú cickányormányos (Podlussány 2010)

Perapion (Perapion) marchicum (Herbst, 1797) - lilás cickányormányos

Perapion (Perapion) oblongum (Gyllenhal, 1839) - fénytelen cickányormányos

Perapion (Perapion) violaceum violaceum (Kirby, 1808) - sóskacickányormányos (BALÁs \& SÁRINGER 1984, DESEÖ in JERMY \& BALÁZs 1990, GoZMÁNy 1979, MER KL etal.2019, PodLUSSÁNY 2009,2010,2014,SZÉNÁsi2012, SZÉNÁsi et al. 2016) - Other names: lórom cickánybogár (DUDICH 1950, GOZM ÁNY 1979); lórom-cickánybogár, sóska-cickánybogár (DESEŐ in JERMY \& BALÁZs 1990)

Phrissotrichum Schilsky, 1901

Schilskyapion Alonso-Zarazaga, 1990

Phrissotrichum (Schilskyapion) rugicolle (Germar, 1817) - serteszőrös cickányormányos 


\section{Pirapion Reitter, 1916}

Pirapion immune (Kirby, 1808) - zanótfúró cickányormányos

Pirapion redemptum (Schatzmayr, 1920) - rekettyeszár-cickányormányos

Protapion Schilsky, 1908

Protapion apricans (Herbst, 1797) - lóheremag-cickányormányos (DESEŐ in Jermy \& BALÁzs 1990, KASza B 1969, MERKL \& Vig 2009, MERKL et al. 2019, Podlussány 2009, 2010, SZÉNÁsi 2012, SzÉNÁsi et al. 2016) - Other names: lóhere cickánybogár (DUDICH 1950); lóheremag-cickánybogár (KASZAB 1969); here-cickánybogár, lóheremag-cickánybogár, vörösheremag-cickánybogár, lóhere-cickánybogár (GOZMÁNY 1979); vörösheremag-cickányormányos (lóheremag-cickánybogár, hosszúorrú cickánybogár, vörösheremagcickánybogár, napotkedvelő cziczkányocska (DESEÖ in JERMY \& BALÁZS 1990); vörösheremag-cickányormányos (HAR MOs et al. 2002)

Protapion assimile assimile (Kirby, 1808) - virágrontó cickányormányos (Deseö in Jermy \& Balázs 1990, Merkl \& SzénÁsi 2018, Podlussány 2010) - Other names: virágrontó lóhereormányos (DUDICH 1950, DUDICH \& LoKsA 1969); vöröshere-magcickánybogár, virágrontó lóhereormányos (GOZMÁNY 1979); vörösheremag-cickányormányos, rövidorrú cickánybogár, hasonló cziczkányocska (DESEŐ in JER MY \& BALÁzs 1990)

Protapion difforme (Germar, 1818) - torzlábú cickányormányos

Protapion dissimile (Germar, 1817) - bunkóscsápú cickányormányos Other names: lóheremag-cickányormányos (DESEÖ in JERMY \& BALÁzs 1990); széleslábú cickányormányos (PoDLUSSÁNY 2009)

Protapion filirostre (Kirby, 1808) - fekete cickányormányos (MER KL 2018, MERKL \& SZÉNÁsi 2018, Podlussány 2009)

Protapion fulvipes fulvipes (Geoffroy, 1785) (= dichroum Bedel, 1885; = flavipes Paykull, 1792) - vadhere-cickányormányos (MERKL 2018, MERKL \& SZÉnÁSI 2018, MER KL et al.2019, PodlusSÁnY 2009, 2010,2014, SZÉNÁSI 2012) Other names: nagyobb vadhereormányos (DUDICH 1950, GozMÁNY 1979); vadhereormányos (Gozmány 1979 KASZAB 1969); fehérheremagcickányormányos (vadhereormányos, nagy vadhereormányos, sárgalábúcickánybogár, fehérheremag-cickánybogár) (DESEÖ in JER MY \& BALÁzs 1990)

Protapion gracilipes (Dietrich, 1857) - piroscsápú cickányormányos

Protapion interjectum interjectum (Desbrochers des Loges, 1895) hegyihere-cickányormányos (MERKL et al. 2019) - Other names: téveszthető cickányormányos (PoDLUSS ÁNY 2010)

Protapion nigritarse (Kirby, 1808) - kis cickányormányos (DESEÖ in JER MY \& BALÁzs 1990, HARMOSetal.2002, MER KL 2018, MER KL \&SZÉNÁ SI2018, MERKL et al. 2019, PodLUSSÁNy 2009, 2010, 2014, SZÉNÁSI 2012, SZÉNÁsi et al. 2016) - 
Other names: kis vadhereormányos (DuDICH 1950); kis vadhereormányos (Deseö in JeRMY \& BALÁzS 1990)

Protapion ononidis (Gyllenhal, 1827) (= ononicola Bach, 1854) - iglicecickányormányos (MERKL \& SzÉnÁsi 2018, MERKL et al. 2019, PodlussánY 2009, 2014, SZÉNÁsI 2012, SZÉNÁsI et al. 2016)

Protapion ruficrus (Germar, 1817) - bércihere-cickányormányos (Podlussány 2009, Merkl et al. 2019)

Protapion schoenherri (Boheman, 1839) - simatorú cickányormányos Other names: Schönherr-cickányormányos (SzÉNÁsı et al. 2016)

Protapion trifolii (Linnaeus, 1768) (= aestivum Germar, 1817) - lóherevirágcickányormányos(MER KL\&SZÉNÁsi2018, MER KLetal.2019,PODLUSSÁNY2009, SZÉNÁsi 2012) - Other names: nyári lóhereormányos (DUDICH 1950); lóherecickánybogár (GYőrFFY 1956, UBRIZSY \& ReICHART 1958, KASZAB 1969); lóherebogár, lóherevirág-cickánybogár, vörösherevirág-cickánybogár, lóherecickánybogár, nyári lóhereormányos (GozMÁNY 1979); vörösherevirágcickányormányos (vöröshere-cickánybogár, here-cickánybogár, lóherevirágcickánybogár, lóhere ápion, luczerna cziczkányocska) (DESEÖ in JERMY \& BALÁzs 1990); vörösherevirág-cickányormányos (HARMos et al. 2002)

Protapion varipes (Germar, 1817) - görbelábú cickányormányos (MER KL \& SZÉNÁsi 2018, MER KL etal.2019, PodlusSÁnY 2009, 2010,2014, SZÉNÁsi 2012) Other names: tarkalábú lóhereormányos (DUDICH 1950); tarkalábú lóherecickányormányos (változólábúcziczkányocska)(DESEöinJERMY \&BALÁzs 1990); tarkalábú lóhere-cickányormányos (HAR MOS et al. 2002)

\section{Protopirapion Alonso-Zarazaga, 1990}

Protopirapion atratulum (Germar, 1817) (= striatum Marsham, 1802) testes cickányormányos

Protopirapion kraatzii (Wencker, 1859) - ólomszürke cickányormányos

Pseudapion Schilsky, 1906

Pseudapion fulvirostre (Gyllenhal, 1833) - fehérmályva-cickányormányos (SzÉNÁsi et al. 2016)

Pseudapion rufirostre (Fabricius, 1775) - vörösorrú cickányormányos Other names: mályvarontó cickánybogár (GOZMÁNY 1979) 


\section{Pseudoperapion Wagner, 1930}

Pseudoperapion brevirostre (Herbst, 1797) - fényesorrú cickányormányos (Merkl 2018, Merkl \& Szénási 2018, Podlussány 2009, 2010, 2014, SZÉNÁsi 2012, SZÉNÁsI et al. 2016)

Pseudoprotapion Ehret, 1990

Pseudoprotapion astragali (Paykull, 1800) - csüdfü-cickányormányos (MERKL \& SZÉNÁsi 2018)

Pseudoprotapion elegantulum (Germar, 1818) - tüskésfejü cickányormányos Other names: lucernabimbó-cickánybogár (GozMÁNY 1979)

Pseudoprotapion ergenense (Becker, 1864) - aranyoszöld cickány-ormányos (MERKL \& SZÉNÁSI 2018)

Pseudostenapion Wagner, 1930

Pseudostenapion simum (Germar, 1817) - nyurga cickányormányos

\section{Rbopalapion Schilsky, 1906}

Rhopalapion longirostre (Olivier, 1807) - mályvarózsa-cickányormányos Other names: mályvamag-cickányormányos (BALÁs \& SÁRINGER 1984, DESEÖ in JERMY \& BALÁzS 1990, KASZAB 1969, MERKL \& Vig 2009); hosszúcsőrü hüvelyész (I. FRIVALDSZKY 1865)

Squamapion Bokor, 1923

Squamapion atomarium (Kirby, 1808) - kakukkfü-cickányormányos (Podlussány 2009, SZÉNÁsi 2012)

Squamapion cineraceum (Wencker, 1864) (= millum Bach, 1854) csupaszorrú cickányormányos

Squamapionelongatum (Germar, 1817) -zsálya-cickányormányos (MERKL \& SZÉnÁsi 2018, PodLUSSÁNY 2009, SZÉNÁSI 2012)

Squamapion flavimanum (Gyllenhal, 1833) - kis menta-cickányormányos (PodlussánY2009)-Othernames:menta-cickányormányos(PoDlussánY2010, SZÉNÁSI et al. 2016)

Squamapion oblivium (Schilsky, 1902) - keskeny cickányormányos 
Squamapion serpyllicola (Wencker, 1864) (= hoffmanni Wagner, 1930) karcsú cickányormányos

Squamapion vicinum (Kirby, 1808) - nagy menta-cickányormányos (Podlussány 2009, SZÉnÁsi 2012)

Stenopterapion Bokor, 1923

Stenopterapion Bokor, 1923

Stenopterapion (Stenopterapion) intermedium (Eppelsheim, 1875) hosszúszőrű cickányormányos (PoDLussánY 2009)

Stenopterapion (Stenopterapion) meliloti (Kirby, 1808) - somkorócickányormányos (Deseö in Jermy \& BALÁzs 1990, Podlussány 2009, SZÉNÁsi 2012, SZÉNÁsI et al. 2016) - Other names: somkóró cickánybogár (Dudich 1950, Dudich \& LoKsa 1969, GozmÁNy 1979); somkórócickánybogár (DESEÖ in JERMY \& BALÁzS 1990)

Stenopterapion (Stenopterapion) tenue (Kirby, 1808) - lucernaszárcickányormányos (DESEő in JeRmy \& BALÁzs 1990, MERKL 2018, MERKL \& SZÉnÁsi 2018, Merkl et al. 2019, Podlussány 2009, 2014, SZÉnÁsi 2012, SZÉNÁsi et al. 2016) - Other names: lucernacickánybogár (DUDICH 1950, GoZMÁNY 1979); lucernaszár-cickánybogár (GoZMÁNY 1979, KASZAB 1969); lucernaszár-cickánybogár, lucerna-szárápion (DESEŐ in JERMY \& BALÁZS 1990)

Synapion Schilsky, 1902

Synapion Schilsky, 1902

Synapion (Synapion) ebeninum (Kirby, 1808) - lakkfényü cickányormányos (PodLUSSÁNy 2009)

Taeniapion Schilsky, 1906

Taeniapion rufulum (Wencker, 1864) - vörhenyes cickányormányos

Taeniapion urticarium urticarium (Herbst, 1784) - csalán-cickányormányos (Harmos et al. 2002, MER KL \& SZÉNÁsi 2018, MER KL et al.2019, Podlussány 2008, 2010, 2014, SzÉNÁsi et al. 2016) - Other names: csaláncickánybogár (Dudich 1950, Gozmány 1979) 
Taphrotopium Reitter, 1916

Taphrotopium Reitter, 1916

Taphrotopium (Taphrotopium) sulcifrons (Herbst, 1797) - ürömcickányormányos

Trichopterapion Wagner, 1930

Trichopterapion holosericeum (Gyllenhal, 1833) - gyertyánmagcickányormányos (BALÁs \& SÁRINGER 1984, MERKL \& VIG 2009, MERKL et al. 2019, PodLuss ÁNy 2009) - Other names: gyertyán-maglikasztó (GYőRFi 1957, Kasza B 1969, Jolsvay et al. 1977, Martinovich 1958)

Nanophyinae Gistel, 1848

Corimaliini Alonso-Zarazaga, 1989

Allomalia Alonso-Zarazaga, 1989

Allomalia quadrivirgata (A. Costa, 1863) - tamariska-füzényormányos

Nanophyini Gistel, 1848

Dieckmanniellus Alonso-Zarazaga, 1989

Dieckmanniellus chevrieri Boheman, 1845 (= helveticus Tournier, 1867) tarkalábú füzényormányos - Other names: Chevrier-füzényormányos (MER KL \& SZÉNÁsI 2018)

Dieckmanniellus gracilis (L. Redtenbacher, 1849) - karcsú füzényormányos (MERKL et al. 2019)

Dieckmanniellus nitidulus (Gyllenhal, 1838) - fogascombú füzényormányos (Dudich 1950, Merkl \& Szénási 2018, Merkl \& Vig 2009, Merkl et al. 2019, Podlussány 2010, SZÉnÁsi 2012, SZÉNÁsi et al. 2016)

\section{Microon Alonso-Zarazaga, 1989}

Microon sablbergi (C. R. Sahlberg, 1835) - tócsahúr-füzényormányos Other names: sárgás tócsahúrormányos (MER KL \& VIG 2009) 
Nanomimus Alonso-Zarazaga, 1989

Nanomimus anulatus (Aragona, 1830) - fekete füzényormányos (Podlussány 2009)

Nanomimus circumscriptus (Aubé, 1864) - nagy füzényormányos (MERKL et al. 2019, Podlussány 2009)

Nanomimus hemisphaericus (Olivier, 1807) - háromszöges füzényormányos (MERKL \& SZÉNÁSi 2018, MERKL et al. 2019)

Nanophyes Schönherr, 1838

Nanophyes brevis brevis Boheman, 1845 - szőrszálas füzényormányos (MERKL et al. 2019, PODLUSSÁNY 2009, SZÉNÁsi 2012, SZÉNÁSI et al. 2016)

Nanophyes globiformis Kiesenwetter, 1864 - gömbölyded füzény-ormányos (MERKL et al. 2019, PodLussány 2010)

Nanophyes globulus (Germar, 1821) - pettyes füzényormányos

Nanophyes marmoratus marmoratus (Goeze, 1777) - simacombú füzényormányos (DUdich 1950, ENDRődi 1971, GozmánY 1979, MERKL \& SzénÁsi 2018, Merkl \& Vig 2009, Merkl et al. 2019, Podlussány 2009, 2010, 2014, SZÉNÁsi 2012, SZÉNÁsI et al. 2016)

Pericartiellus Alonso-Zarazaga, 1989

Pericartiellus telephii (Bedel, 1900) (= maculipes Rossi, 1793) - varjúhájfüzényormányos

CURCULIONIDAE Latreille, 1802

Bagoinae C. G. Thomson, 1859

Bagous Germar, 1817

Bagous Germar, 1817

Bagous (Bagous) bagdatensis Pic, 1904 (= wagneri Dieckmann, 1964) (Podlussány 1998) - bagdadi víziormányos (PoDlussánY 2007, SZÉNÁsi 2012, SZÉNÁSI et al. 2016)

Bagous (Bagous) binodulus (Herbst, 1795) - kétpúpú víziormányos (Podlussány 2007, SzÉnÁsi 2012)

Bagous (Bagous) glabrirostris (Herbst, 1795) - kolokán-víziormányos (Merkl \& SzÉnÁsi 2018, Podlussány 2007, 2009, SzÉNÁsi 2012) - Other names: marti ormányos (DUDICH 1950, DUDICH \& LoKSA 1969, ENDRöDI 1971) 
Bagous (Bagous) lutulentus (Gyllenhal, 1813) (= nigritarsis C. G. Thomson, 1865) - zsurló-víziormányos (PodLussánY 2007, 2009)

Bagous (Bagous) puncticollis Boheman, 1845 - horpadt víziormányos (Podlussány 2007, SZÉNÁsi 2012, SZÉNÁsi et al. 2016)

Bagous (Bagous) robustus H. Brisout de Barneville, 1863 - vaskos víziormányos - Other names: vaskos hídőrormányos (PoDlussánY 2007, SZÉNÁsi et al. 2016)

Bagous (Bagous) subcarinatus Gyllenhal, 1836 - tócsagaz-víziormányos (Mer Kl \& SZÉnÁsi 2018, Mer Kl \& Vig 2009, Mer Kl et al. 2019, PodlussánY 2007, SZÉNÁSI 2012, SZÉNÁsi et al. 2016)

\section{Macropelmus Dejean, 1821}

Bagous (Macropelmus) argillaceus Gyllenhal, 1836 - lakkfényű víziormányos (MERKL \& SZÉNÁSI 2018) - Other names: lakkfényes víziormányos (Podlussány 2007, SzÉNÁsi 2012, SzÉNÁsi et al. 2016)

Bagous (Macropelmus) bulgaricus Angelov, 1989 (György \& Podlussány 2005) - bolgár víziormányos

Bagous (Macropelmus) claudicans Boheman, 1845 (GYÖRgY \& PodLussánY 2005) - foltos víziormányos (PoDluss ÁNy 2007)

Bagous (Macropelmus) collignensis (Herbst, 1797) - süllöhínár-víziormányos (MER KL \& SZÉNÁsi 2018, PodLUSSÁNy 2007, SZÉNÁsi 2012)

Bagous (Macropelmus) czwalinai Seidlitz, 1891 - foltos víziormányos

Bagous (Macropelmus) dieckmanni Gratshev, 1993 (GYörgY \& PoDLussánY 2005) - fényes víziormányos

Bagous (Macropelmus) elegans (Fabricius, 1801) (Fig. 4) - ékes víziormányos

Bagous (Macropelmus) frit (Herbst, 1795) - vidrafü-víziormányos

Bagous (Macropelmus) friwaldszkyi Tournier, 1874 - Frivaldszkyvíziormányos (PoDlussánY 2007)

Bagous (Macropelmus) geniculatus (Hochhuth, 1847) - széles békaszőlövíziormányos

Bagous (Macropelmus) limosus (Gyllenhal, 1827) - gödröshátú vízior mányos (Podlussány 2007)

Bagous (Macropelmus) longitarsis C. G. Thomson, 1868 - hosszúkarmú víziormányos (PodLUssáNY 2007)

Bagous (Macropelmus) lothari Caldara et O’Brien, 1998 (GYöRgY \& PodLussány 2005) - homályos víziormányos

Bagous (Macropelmus) lutosus (Gyllenhal, 1813) - karcsú békaszőlővíziormányos - Other names: békabuzogány-víziormányos (PoDLussáNY 2007, 2009, SZÉNÁsi et al. 2016)

Bagous (Macropelmus) lutulosus (Gyllenhal, 1827) - szittyó-víziormányos 
Bagous (Macropelmus) majzlani (Kodada, Holecová et Behne, 1992) harmatkása-víziormányos (MER KL \& VIG 2009, MER KL \& SzÉNÁsi 2018)

Bagous (Macropelmus) nodulosus Gyllenhal, 1836 - négypúpú víziormányos (Merkl \& SZÉnÁsi 2018, Podlussány 2007, SzÉnÁsi 2012, SzÉnÁsi et al. 2016)

Bagous (Macropelmus) petro (Herbst, 1795) - rence-víziormányos (SzÉNÁsI 2012, SZÉNÁsI et al. 2016)

Bagous (Macropelmus) rotundicollis Boheman, 1845 - tavirózsa-víziormányos

Bagous (Macropelmus) rufimanus Péricart, 1989 (GYÖRgY \& PodlussánY 2005) - sulyom-víziormányos (Podlussány 2007, SZÉNÁsi et al. 2016)

Bagous (Macropelmus) tempestivus (Herbst, 1795) - boglárka-víziormányos (MERKL \& SZÉnÁsi 2018, Podlussány 2007, 2009)

Bagous (Macropelmus) tubulus Caldara et O'Brien, 1994 (= angustus Silfverberg, 1977; cylindrus Paykull, 1800) - ecsetpázsit-víziormányos (Podlussány 2007)

Bagous (Macropelmus) validus Rosenhauer, 1847 - virágkáka-víziormányos (MERKL \& SZÉNÁsi 2018) - Other names: tömör víziormányos (PODLUSSÁNY 2007); vaskos víziormányos (SzÉNÁsı 2012)

Hydronomus Schönherr, 1825

Hydronomusalismatis (Marsham, 1802) - hídőr-víziormányos (PoDlussánY 2007) - Other names: hídőrormányos (DUDICH 1950)

Brachycerinae Billberg, 1820

Brachycerini Billberg, 1820

Brachycerus Olivier, 1789

Brachycerus foveicollis Gyllenhal, 1833 - szögletes ragyásormányos (MERKL \& VIG 2009, SZÉNÁsI et al. 2016) - Other names: szögletes ormányos (DUDICH 1950)

Erirhinini Schönherr, 1825

Bagoopsis Faust, 1881 2007)

Bagoopsisglobicollis(Fairmaire, 1863) - szürkeártériormányos (PODLUSSÁNY 


\section{Grypus Germar, 1817}

Grypus brunnirostris (Fabricius, 1792) - kis zsurlóormányos

Grypus equiseti (Fabricius, 1775) - nagy zsurlóormányos (MERKL \& VIG 2009, PodLuss ÁNY 2007, 2009) - Other names: zsurlóormányos (DUDICH 1950, GoZMÁNY 1979)

Icaris Tournier, 1874

Icarissparganiisparganii (Gyllenhal, 1835) - széles békabuzogány-ormányosOther names: békabuzogány-ormányos (DUDICH 1950, HaRmos et al. 2002, MERKL et al. 2019)

Lepidonotaris Zumpt, 1929

Lepidonotarispetax (C. R. Sahlberg, 1823) (GYÖRGY \& PoDLUSSÁNY 2005) alföldi nádormányos

Notaris Germar, 1817

Notaris acridula (Linnaeus, 1758) - közönséges sásormányos (MERKL \& SZÉNÁSI 2018)

Notaris aterrima (Hampe, 1850) - rejtettpajzsocskás sásormányos

Notaris maerkeli (Boheman, 1843) (SZÉNÁsI 2014) - szurokszínü sásormányos - Other names: Maerkel-ormányos (PoDLussánY 2009)

Notaris scirpi (Fabricius, 1792) - kákafúró sásormányos (MER KL \& SZÉNÁsI 2018) - Other names: kákaormányos (Dudich 1950, Dudich \& LoKsa 1969, EndRődi 1971, Gozmány 1979, MERKL \& Vig 2009, Podlussány 2007, SZÉNÁsI 2012, SZÉNÁsI et al. 2016)

\section{Thryogenes Bedel, 1884}

Thryogenes festucae (Herbst, 1795) - közönséges nádormányos (MER KL \& SZÉnÁsi 2018, MER KL et al. 2019, Podlussány 2007, SzÉnÁsi 2012) - Other names: nádormányos (DUdich 1950, GozmáNy 1979)

ThryogenesfioriiZumpt, 1928(=atrirostrisLohse, 1992)(PoDLuss ÁNY 1998)sárga nádormányos (PoDLUSSÁNY 2007)

Thryogenes nereis (Paykull, 1800) - nádtippanormányos (PoDLUssáNY 2007) 
Thryogenes scirrhosus (Gyllenhal, 1835) - karcsú békabuzogány-ormányos (MERKL \& SZÉNÁSI 2018, Podlussány 2007, SZÉNÁsi et al. 2016)

Tournotaris Alonso-Zarazaga et Lyal, 1999

Tournotaris bimaculata (Fabricius, 1787) - fürészeslábú gyékényormányos

Tournotaris granulipennis (Tournier, 1874) (PoDlussáNy 1998) - érdes gyékényormányos

Tanysphyrini Gistel, 1848

Stenopelmus Schönherr, 1835

Stenopelmus rufinasus Gyllenhal, 1835 (Podlussány 2001b) moszatpáfrány-ormányos (MER KL \& VIG 2009, SZÉNÁsI et al. 2016)

\section{Tanysphyrus Germar, 1817}

Tanysphyrus ater Blatchley, 1928 (= makolskii Smreczyński, 1957) májmohaormányos (MER KL \& VIg 2009, PodLUSSÁNy 2007, SZÉNÁsi 2012)

Tanysphyrus lemnae (Paykull, 1792) - békalencse-ormányos (DUDICH 1950, DUdich \&LoKsa 1969, GozMÁNy 1979, HaR mos etal.2002, Jolsvay etal.1977, Merkl \& SzénÁsi 2018, Merkl \& Vig 2009, Podlussány 2007, 2009, 2010, SZÉNÁSI 2012, SZÉNÁSI et al. 2016)

Conoderinae Schönherr, 1833

BARIDITAE Schönherr, 1836

Apostasimerini Schönherr, 1844

Limnobaris Bedel, 1885

Limnobaris dolorosa (Goeze, 1777) (= pilistriata Stephens, 1831) - szálkás báris (Merkl \& SzénÁsi 2018, Merkl \& Vig 2009, Merkl et al. 2019, PodLUSSÁNy 2007, SZÉNÁsi 2012, SZÉNÁsi et al. 2016)

Limnobarist-album (Linnaeus, 1758) - réti báris (DUDICH 1950, DUDICH \& LoKsa 1969, Gozmány 1979, Jolsvay et al. 1977, MERKL \& SzÉnÁsi 2018, MERKL et al. 2019, PodLUSSÁNY 2007, 2009, 2010, SZÉNÁsi 2012) 
Baridini Schönherr, 1836

Aulacobaris Desbrochers des Loges, 1892

Aulacobaris angusta angusta (Brullé, 1832) - keskeny báris (PodLussánY 2007)

Aulacobaris caerulescens caerulescens (Scopoli, 1763) - fémes repcebáris (MERKL \& SZÉNÁSI 2018)

Aulacobaris chlorizans (Germar, 1824) - kék káposztabáris (PoDlussánY 2007) - Other names: kék káposztabarkó (Jolsvay et al. 1977, KAszaB 1969, Manninger 1960, Péterfi 1958, Ubrizsy \& Reichart 1958); nagy repcebarkó (MANNINGER 1960); káposztaszár-ormányos (BALÁs \& SÁRINGER 1984); keskeny pocokfogú-ományos, kis káposztabarkó, kék káposztabarkó nagy repcebarkó, káposztaszárormányos, repce-pocokbogár (GozMÁNY 1979); káposztaszár-ormányos (kék káposztabarkó, nagy repcebarkó, repce pocokbogár) (SÁRINGER in JERMY \& BALÁzS 1990)

Aulacobaris cuprirostris cuprirostris (Fabricius, 1787) (KRÁTKÝ \& PODLUSSÁNY 2008) - rezes báris - Other names: rezesorrú pocokfogú-ormányos, pirosorrú pocokbogár (GoZMÁNY 1979)

Aulacobaris gudenusi (Schultze, 1901) - kék zsomborbáris (PoDlussány \& KUTASI 2011)

Aulacobaris janthina (Boheman, 1836) (SzÉNÁsı 2018) - nyúlánk báris (SZÉNÁSI 2018)

Aulacobaris kaufmanni (Reitter, 1897) (György \& Podlussány 2005) holdviolabáris

Aulacobaris lepidii (Germar, 1824) - kékes zsázsabáris - Other names: zsázsabáris (Dudich 1950, Dudich \& LoKsa 1969, Podlussány 2007, 2014, SzÉNÁsI 2012); káposztabarkó (UBRIZsY \& REICHART 1958); zsázsaormányos (BALÁs \& SÁRINGER 1984); zsázsaormányos (zsázsabáris) (JolsvaY et al. 1977, GOZMÁNy 1979, SÁRINGER in JERMY \& BALÁzs 1990)

Aulacobaris picicornis picicornis (Marsham, 1802) - közönséges rezedabáris (MERKL \& SZÉNÁSI 2018) - Other names: rezedabáris (PoDLUSSÁNy 2007)

Baris Germar, 1817

Baris analis analis (Olivier, 1791) - vörösfarú báris (PoDLussÁNY 2007, SZÉNÁsI 2012)

Barisartemisiae(Herbst, 1794)-feketeürömbáris(MER KL \&SZÉNÁsı 2018)Other names: ürömbáris (DUDICH 1950, GozmÁNY 1979, HARMOs et al. 2002, Podlussány 2010) - Other names: közönséges ürömbáris (PODLUSSÁnY 2007, 2009, 2014, SZÉNÁSI 2012) 
Cosmobaris Casey, 1920

Cosmobaris scolopacea (Germar, 1819) - pikkelyes báris (DUDICH 1950, Dudich \& LoKsa 1969, Harmos et al. 2002, Jolsvay et al. 1977, MerkL \& SZÉNÁsi 2018, MER KL \& Vig 2009, MERKL et al. 2019, SZÉNÁSI 2012)

Eremobaris Zaslavskij, 1956

Eremobaris picturata (Ménétriés, 1849) - tarka báris

Labiaticola Alonso-Zarazaga et Lyal, 1999

Labiaticola atricolor (Boheman, 1844) - hasznostisztesfü-báris (MER KL \& SZÉNÁSI 2018)

Malvaevora Zaslavskij, 1956

Malvaevora timida timida (Rossi, 1792) - fekete mályvabáris - Other names: nagy mályvaormányos (BALÁs \& SÁRINGER 1984, SÁR INGER in JERMY \& BAlázs 1990); mályvabáris (Merkl \& Vig 2009, Podlussány 2007, 2014, SZÉNÁSI 2012)

\section{Melanobaris Alonso-Zarazaga et Lyal, 1999}

Melanobaris atramentaria (Boheman, 1836) - repcsénybáris - Other names: fekete báris (MER KL 2018, MERKL \& SZÉNÁsI 2018, PODLUSSÁNY 2007, SZÉNÁsI 2012)

Melanobaris carbonaria (Boheman, 1836) - nagy rekenyőbáris - Other names: nagy káposztaormányos (KASZA B 1969, Jolsvay et al. 1977, BALÁs \& SÁRINGER 1984, SÁR INGER in JERMY \& BALÁZs 1990); nagy báris (PODLUSSÁNY 2007)

Melanobaris dalmatina (H. Brisout de Barneville, 1870) - dalmát báris

Melanobaris laticollis (Marsham, 1802) - lakkfényü káposztabáris (Podlussány 2007, MERKL 2018) - Other names: szélesnyakú báris (Dudich 1950); fekete káposztabarkó (KASZA B 1969, MANNINGER 1960, PÉTERFI 1958); fekete káposztabarkó (káposztagyökér-barkó, szélesnyakú báris, szurokszínü pocokbogár) (JolsvaY et al. 1977); káposztatő-ormányos (BALÁs \& SÁRINGER 1984); szélesnyakú báris, fekete káposztabarkó, káposztagyökér-barkó, káposztatő-ormányos, szurokszínű pocokbogár, fekete pocokfogú-ormányos 
(GoZMÁNY 1979); káposztatő-ormányos (szurokszínű pocokbogár, szélesnyakú baris, fekete káposztabarkó, káposztagyökérbarkó) (SÁRINGER in JERMY \& BALÁzs 1990); káposzta-gyökérbarkó (MANNINGER 1960)

Melanobaris morio (Boheman, 1844) (KRÁtKÝ \& PodLussánY 2008) vöröscsápú rezedabáris - Other names: vöröscsápú báris (PODLUSsánY \& KuTASI 2011)

Melanobaris steppensis (Roubal, 1935) - gödörkéstorú báris

Madarini Jekel, 1865

Cucubaris Alonso-Zarazaga, 2005

Cucubaris villae (Comolli, 1837) - földitökbáris (PoDLussánY 2007)

Ceutorhynchitae Gistel, 1848

Amalini Wagner, 1936

Amalus Schönherr, 1825

Amalus scortillum (Herbst, 1795) (= haemorrhous Herbst, 1795) - barna keserüfü-ormányos (MER KL et al. 2019, PODLUSSÁNY 2009, 2014, SZÉNÁsi 2012, SZÉNÁsi et al. 2016) - Other names: csarabormányos (DUDICH 1950)

Ceutorhynchini Gistel, 1848

Amalorrhynchus Reitter, 1913

Amalorrbynchus melanarius (Stephens, 1831) - kányafürontó ormányos Other names: fekete vízitormabarkó (GozmáNY 1979)

Calosirus C. G. Thomson, 1859

(= Sirocalus Heyden, 1906)

Calosirus terminatus (Herbst, 1795) - petrezselyemormányos (PodLussánY 2009, Podlussány 2009, 2010, SzÉnÁsi 2012) - Other names: fekete répaormányos (GOZMÁNY 1979) 
Ceutorhynchus Germar, 1824 (= Ceutorrhynchus Gemminger, 1871; Neosirocalus Neresheimer et Wagner, 1944)

Ceutorhynchus aeneicollis Germar, 1824 - aranyoszöld ceutormányos Other names: zsázsa-ceutormányos (PoDlussáNY 2007); zsázsaormányos (SzÉNÁsi et al. 2016)

Ceutorhynchus alliariae $\mathrm{H}$. Brisout de Barneville, 1860 - pirostappancsos ceutormányos (PoDLussáNY 2007) - Other names: pirostappantyús ormányos (Podlussány 2010, SZÉNÁsi et al. 2016)

Ceutorhynchus arator Gyllenhal, 1837 - tátorján-ceutormányos

Ceutorhynchus assimilis (Paykull, 1792) (= pleurostigma Marsham, 1802) (PodlussáNy 2001b) - repcegyökér-ceutormányos (MERKL \& SZÉNÁsi 2018, Podlussány 2007, 2014, SZÉNÁsi 2012, SZÉNÁsi et al. 2016) - Other names: repcegyökér-ormányos (DUDICH 1950); repcegubacsbarkó (ENDRÖDI 1968, KASZAB 1969, MANNINGer 1960, Ubrizsy \& ReICHART 1958); repcegubacsbarkó, repcegyökér-ormányos, gubacsormányos, gubacsbarkó, gubacslakó káposztabarkó, káposztagubacs-barkó (GozMÁNY 1979); káposztagubacsbarkó (MANNINGER 1960); gubacsbarkó (KADOcSA 1923); gubacslakó káposztabarkó (JABLONOWSKI 1914); gubacsormányos (gubacsbarkó, gyökérgubacsormányos, káposzta-gyökérbarkó, káposzta-gubacsbarkó, gubacslakó káposztabarkó, repce-gubacsbarkó, repce-gyökérbarkó) (SÁRINGER in JERMY \& BALÁzs 1990); gubacsormányos (BALÁs \& SÁRINGER 1984, HaR mos et al. 2002)

Ceutorhynchus atomus Boheman, 1845 - szörös ceutormányos (PoDlussáN Y 2007, 2009)

Ceutorhynchus barbareae Suffrian, 1847 - borbálafü-ceutormányos

Ceutorhynchus canaliculatus C. N. F. Brisout de Barneville, 1869 barázdáshátú ceutormányos - Other names: barázdás ceutormányos (Podlussány 2007, SzÉnÁsi 2012)

Ceutorhynchuscarinatus Gyllenhal, 1837 - tarsóka-ceutormányos(MER KL \& SZÉNÁsI 2018, PodLUSSÁNy 2007, 2014, SZÉNÁsi 2012, SZÉNÁsI et al. 2016)

Ceutorhynchus chalybaeus Germar, 1824 (= timidus Weise, 1883) - fémes ceutormányos (MER KL \& SZÉNÁsi 2018, PodLUSSÁNy 2007, 2010, 2014)

Ceutorhynchus chlorophanus Rouget, 1857 - zöldes repcsény-ceutormányos (MERKL 2018, \& SZÉNÁsI 2018)

Ceutorhynchus coarctatus Gyllenhal, 1837 - ráspolyostorú ceutormányos (MER KL \& SZÉNÁsi 2018, PodlusSÁNy 2007, SZÉNÁSI 2012)

Ceutorhynchus cochleariae (Gyllenhal, 1813) - fekete kakukktormaceutormányos - Other names: kakukktorma-ceutormányos (PoDLUSSÁNY 2007)

Ceutorhynchus constrictus (Marsham, 1802) - gömbölyü ceutormányos (Podlussány 2007)

Ceutorhynchus contractus (Marsham, 1802) (= minutus Reich, 1797; = pallipes Crotch, 1866) - vadrepce-ceutormányos (MERKL 2018, MERKL \& SZÉNÁsi 2018, MER KL et al. 2019, PODLUSSÁNy 2007, SZÉNÁsi et al. 2016) 
Ceutorbynchus dubius C. N. F. Brisout de Barneville, 1883 - szürkés hamukaceutormányos - Other names: ritka hamuka-ceutormányos (PoDlussánY 2007)

Ceutorhynchus erysimi (Fabricius, 1787) (= henschi Schultze, 1903) - kis repcsény-ceutormányos (MERKL 2018, MERKL \& SzÉNÁsi 2018, MERKL et al. 2019, Podlussány 2009, 2014, SzÉNÁsi 2012, SZÉnÁsi et al. 2016) Other names: kis repcsényormányos (DUDICH 1950, DUDICH \& LoKsA 1969, Gozmány 1979, HaRmos et al. 2002, Podlussány 2010)

Ceutorhynchus fallax Boheman, 1845 - fehérpettyes ceutormányos

Ceutorhynchus fulvitarsis Gougelet et $\mathrm{H}$. Brisout de Barneville, 1860 (Podlussány 2001a) - nyugati ceutormányos

Ceutorbynchus gallorhenanus F. Solari, 1949 - liszteshátú ceutormányos (PodLussánY 2007)

Ceutorbynchus granulicollis C. G. Thomson, 1865 (= gerbardti Schultze, 1899) (Podlussány 2001b) - szemcséstorú ceutormányos

Ceutorhynchus griseus C. N. F. Brisout de Barneville, 1869 - hamvas ceutormányos (PodlussánY 2007, 2010, SZÉNÁsi et al. 2016)

Ceutorhynchus hampei C. N. F. Brisout de Barneville, 1869 - szélestorú ceutormányos - Other names: Hampe-ceutormányos (PoDlussánY 2007, SZÉNÁSI 2012)

Ceutorhynchus hirtulus Germar, 1824 - lúdfü-ceutormányos (MER KL 2018, Merkl \& SZÉnÁsi 2018, MER KL et al. 2019, PodLusSÁnY 2007, 2014, SZÉnÁsi 2012, SZÉNÁsI et al. 2016)

Ceutorhynchusignitus Germar, 1824- kék hamuka-ceutormányos(MER KL \& VIG 2009) - Other names: kis hamuka-ceutormányos (PodLUSSÁNY 2007, SZÉNÁsI 2012)

CeutorhynchusinaffectatusGyllenhal, 1837-estike-ceutormányos(MER KL \& SZÉnÁsi 2018, PodLUSSÁNY 2007, SZÉNÁsi 2012)

Ceutorhynchus interjectus Schultze, 1903 - magaszsombor-ceutormányos (PodLussánY 2007)

Ceutorhynchus levantinus Schultze, 1898 - keskeny ceutormányos (Podlussány 2007)

Ceutorbynchusmerkli Korotyaev, 2001 (PoDlussán Y 2001b) - útszélizsázsaceutormányos (MERKL \& VIG 2009)

Ceutorhynchus moraviensis (Dieckmann, 1966) - morva ceutormányos

Ceutorhynchus nanus Gyllenhal, 1837 (= angustulus Gyllenhal, 1837; = trivialis Faust, 1884) - homoki ceutormányos (MERKL \& SzÉNÁsI 2018) Other names: törpe ceutormányos (PoDLussÁNY 2007, SZÉNÁsI 2012)

Ceutorhynchusnapi Gyllenhal, 1837 - széles repce-ceutormányos (PoDLussánY 2007, SZÉNÁsi 2012) - Other names: nagy repceormányos (MANNINGER 1960); nagy káposztaormányos (BALÁs \& SÁRINGER 1984); nagy repceormányos, nagy káposztaormányos (GOZMÁNY 1979); nagyrepceormányos(nagy káposztaormányos, nagy káposztabarkó) (SÁRINGER in JERMY \& BALÁZS 1990) 
CeutorhynchusnigritulusSchultze, 1897-ikravirág-ceutormányos(MER KL \& SZÉNÁsi 2018, PoDLUSSÁNY 2007, SZÉNÁsI 2012)

Ceutorhynchusniyazii Hoffmann, 1957 (=vindobonensis Dieckmann, 1966) pirosorrú ceutormányos (MERKL \& SzÉNÁsI 2018) - Other names: Niyaziceutormányos (SZÉNÁSI 2012)

Ceutorhynchus obstrictus (Marsham, 1802) - repcebecő-ceutormányos (Merkl \& Szénási 2018, Merkl et al. 2019, Podlussány 2007, 2009, 2014, SzÉNÁsi 2012) - Other names: repcebecő-ormányos (DUdICH 1950, MANNINGer 1960, ENDRődi 1968, Harmos et al. 2002, Podlussány 2010); repceormányos (KASZA B 1969); repce batlaorrú-ormányos, repcebecő-ormányos, repceszár-ormányos, repceormányos (GoZMÁNY 1979); repcebecő-ormányos (repceormányos) (SÁRINGER in JERMY \& BALÁzs 1990)

Ceutorhynchus pallidactylus (Marsham, 1802) (= quadridens Panzer, 1794) szőrös repce-ceutormányos (MERKL 2018, MERKL \& SzÉNÁsI 2018, Merkl et al. 2019, Podlussány 2007, 2014) - Other names: keresztes gyökérormányos (DUDICH 1950); foltos káposztabarkó (MANNINGER 1960); foltos káposztaormányos (MANNINGER 1960); repceormányos (ENDRőDI 1968); repcerügyormányos (DUDICH \& Loksa 1969); repcegyökérormányos, repceormányos, repceszárbarkó, keresztes gyökérormányos, repceszárormányos (GoZMÁNY 1979); repceszárbarkó(BALÁs 1963, KA SZA B 1969, MAN NINGER 1960, UBRIZSY \& REICHART 1958); repceszár-ormányos (keresztesgyökérormányos, repceormányos, repceszárbarkó, torzskukac) (SÁRINGER in JERMY \& BALÁzS 1990); repceszárormányos (BALÁs \& SÁRINGER 1984, HARMOs et al. 2002); repce-ceutormányos (Podlussány 2007, SzÉNÁsI 2012, SZÉNÁsI et al. 2016)

Ceutorhynchus pandellei C. N. F. Brisout de Barneville, 1869 - fogasírceutormányos

Ceutorhynchus parvulus C. N. F. Brisout de Barneville, 1869 - kis zsázsaceutormányos (MERKL \& SzÉNÁsi 2018) - Other names: aprócska zsázsaceutormányos (PoDLUSSÁNY 2007, SZÉNÁsI 2012)

Ceutorhynchus pectoralis Weise, 1895 - fehérfoltos kakukktormaceutormányos - Other names: kakukktorma-ceutormányos (PoDLUSSÁNY 2007)

Ceutorhynchus pervicax Weise, 1883 - zöld vízitorma-ceutormányos (Podlussány 2007)

Ceutorhynchus picitarsis Gyllenhal, 1837 - fekete káposzta-ceutormányos (MERkL \& SzÉnÁsi 2018, Podlussány 2014) - Other names: fekete káposztaormányos (GozmÁNy 1979, BALÁs \& SÁRINGER 1984, HaRmos et al. 2002, SÁringer in JERMY \& BALÁzs 1990, SzÉNÁsi et al. 2016); fekete repceormányos (GOZMÁNY 1979, MANNINGER 1960); fekete káposztabarkó (GozmÁny 1979, MANninger 1960); káposzta-ceutormányos (PodlussánY 2007)

Ceutorhynchus posthumus Germar, 1824 - rejtőke-ceutormányos (Podlussány 2007) - Other names: rejtőke-ormányos (PodLussány 2010) 
Ceutorhynchus pulvinatus Gyllenhal, 1837 - kis zsombor-ceutormányos (Merkl \& SZÉnÁsi 2018, Podlussány 2007, SzénÁsi 2012) - Other names: kis zsomborormányos (DUDICH 1950)

Ceutorhynchus puncticollis Boheman, 1845 - pontozott ceutormányos (MERKL 2018, MERKL \& SZÉNÁsi 2018) - Other names: pontozott hamukaceutormányos (PodlussánY 2007, 2014)

Ceutorhynchus pyrrhorhynchus (Marsham, 1802) - vörösorrú ceutormányos

Ceutorhynchus rapae Gyllenhal, 1837 - nagy repcsény-ceutormányos (PodLUSSÁNY 2007, SzÉNÁsi 2012) - Other names: nagy repcsényormányos (Dudich 1950, Dudich \& LoKsa 1969, GozmáNy 1979); kenderormányos (nagy repcsényormányos) (NAGY in JeRMY \& BALÁzs 1990)

Ceutorhynchus rhenanus (Schultze, 1895) - szürke repcsény-ceutormányos

Ceutorhynchus roberti Gyllenhal, 1837 - kányazsombor-ceutormányos (PodlussáNY 2014) - Other names: kányazsombor-ceutormányos (SzÉNÁsI et al. 2016)

Ceutorhynchus scapularis Gyllenhal, 1837 - kányafü-ceutormányos

Ceutorhynchus scrobicollis Neresheimer et H. Wagner, 1924 - gödröstorú ceutormányos (PODLUSSÁNY 2007) - Other names: erdei ceutormányos (MER KL \& SZÉNÁsI 2018); gödröstorú ormányos (PoDluSSÁNY 2010, SZÉNÁsI et al. 2016)

Ceutorhynchus similis C. N. F. Brisout de Barneville, 1869 - kis tarsókaceutormányos

Ceutorhynchus sisymbrii (Dieckmann, 1966) - szürke zsomborceutormányos - (MERKL \& SZÉNÁsI 2018)

Ceutorhynchus sophiae Gyllenhal, 1837 - sebforrasztófü-ceutormányos (MER KL \& SZÉNÁSI 2018, MER KL et al.2019, PodLUSSÁNy 2007, 2014, SZÉNÁsi 2012)

Ceutorhynchus striatellus Schultze, 1900 (= angustus Dieckmann et Smreczyński, 1972) - kis ternye-ceutormányos (MERKL \& SzÉNÁsi 2018, PodLUSSÁNY 2007)

Ceutorhynchus subpilosus C. N. F. Brisout de Barneville, 1869 (MERKL et al. 2012) - szürke ternye-ceutormányos - Other names: ternye-ceutormányos (MERKL et al. 2012)

Ceutorhynchus sulcatus C. N. F. Brisout de Barneville, 1869 - fémeskék ceutormányos - Other names: álomszép ceutormányos (PoDLUSSÁNy 2007); fémkék ceutormányos (MER KL \& SZÉNÁSI 2018)

Ceutorhynchus sulcicollis (Paykull, 1800) - nagy zsombor-ceutormányos (PodlussáNy 2007) - Other names: nagyobb zsomborormányos (DUDICH 1950, GozmáNY 1979); repce batlaorrú ormányosa (GozmánY 1979)

Ceutorhynchus syrites Germar, 1824 - szír ceutormányos (MERKL \& SZÉNÁSI 2018, PodLuSSÁNY 2007) - Other names: gomborkaormányos (GoZMÁNY 1979); szíriai ceutormányos (SZÉNÁsI 2012) 
Ceutorhynchus talickyi Korotyaev, 1980 (= strejceki Dieckmann, 1981) pusztai ceutormányos (MERKL \& SzÉNÁsi 2018) - Other names: Talickyceutormányos (PoDLussánY 2007)

Ceutorhynchus turbatus Schultze, 1903 - pálcikapikkelyủ ceutormányos Other names: zsázsa-ceutormányos (PoDLUSSÁNY 2007, SZÉNÁsI 2012, SZÉNÁsI et al. 2016)

Ceutorhynchus typhae (Herbst, 1795) (= floralis Paykull, 1792) pásztortáska-ceutormányos (MERKL \& SZÉNÁsI 2018, MERKL et al. 2019, Podlussány 2007, 2009, 2014, SzÉNÁsi 2012, SzÉNÁsi et al. 2016) - Other names: pásztortáskaormányos (DUdich 1950, Dudich \& LoKsA 1969, PODLUSSÁNY 2010)

Ceutorhynchus unguicularis C. G. Thomson, 1871 - hosszúkarmú ceutormányos (MERKL \& SZÉNÁsi 2018, MERKL et al. 2019) - Other names: karmos ceutormányos (PodLussánY 2007)

Ceutorhynchus varius Rey, 1895 (KRÁtKÝ \& Podlussány 2008) - fekete ceutormányos (MER KL \& SZÉNÁsI 2018)

Ceutorhynchus wagneri Smreczyński, 1937 - négyzetes ceutormányos

\section{Coeliastes Weise, 1883}

Coeliastes lamii (Fabricius, 1792) - árvacsalán-ormányos (DuDICH 1950, Harmos et al. 2002, MERKL 2018, Merkl et al. 2019, PodLuss Ány 2007, 2009, 2010, 2014, SZÉNÁsi et al. 2016)

\section{Coeliodes Schönherr, 1837}

Coeliodes proximus Schultze, 1895 - szurkosorrú gömböcormányos (Podlussány 2007, 2014)

Coeliodes rana (Fabricius, 1787) (= dryados Gmelin, 1790; = quercus Fabricius, 1787) - cser-gömböcormányos (MERKL \& VIG 2009, PODLUSSÁNY 2007, SZÉNÁsi et al. 2016) - Other names: cserormányos (GozMÁNY 1979)

Coeliodes ruber (Marsham, 1802) - vörös gömböcormányos (PoDlussÁNY 2007)

Coeliodes transversealbofasciatus (Goeze, 1777) (= cinctus Fourcroy, 1785; = erythroleucos Gmelin, 1790) - fehéröves gömböcormányos (PoDLUSSÁNY 2007, SZÉNÁsi et al. 2016)

Coeliodes trifasciatus Bach, 1854 - tölgy-gömböcormányos (PodLussánY 2007) 
Coeliodinus Dieckmann, 1972

Coeliodinus rubicundus (Herbst, 1795) - nyár-gömböcormányos

Datonychus H. Wagner, 1944

Datonychus angulosus (Boheman, 1845) - kenderkefü-ormányos (PoDlussánY 2007)

Datonychus arquata (Herbst, 1795) - vízipeszérce-ormányos (MERKL \& SZÉnÁsi 2018, PodLUSSÁNY 2007, 2009, 2010, SZÉNÁsi 2012)

Datonychus derennei (Guillaume, 1936) (= magnini Hoffmann, 1939) (Podlussány 2001b) - szurokfüormányos (Podlussány 2007)

Datonychus melanostictus (Marsham, 1802) - nyúlánk mentaormányos (MER KL \& SZÉNÁSI 2018) - Other names: mentaormányos (PodlusSÁNY 2007, 2009, 2010, SZÉNÁsI 2012, SzÉNÁsI et al. 2016)

Datonychus paszlavszkyi (Kuthy, 1890) - fehérfoltos zsályaormányos Other names: Paszlavszky-ormányos (PoDlussány 2007, SzÉnÁsi et al. 2016)

Datonychus scabrirostris (Hochhuth, 1847) (=frivaldszkyi Schultze, 1897) halványfoltos tisztesfüormányos

Datonychus transsylvanicus (Schultze, 1897) - erdélyi tisztesfüormányos

Datonychus urticae (Boheman, 1845) - erdei tisztesfüormányos (PodLusSÁnY 2007)

Ethelcus Reitter, 1916

Ethelcus denticulatus (Schrank, 1781) - pipacsgyötrő ormányos (PoDlussánY 2007) - Other names: barna mákgyökérormányos (BALÁs \& SÁRINGER 1984); barna mák-gyökérormányos (barna mákgyökérbarkó) (SÁRINGER in JERMY \& BALÁzS 1990)

Eucoeliodes Smreczyński, 1974

Eucoeliodes mirabilis (A. Villa et G. B. Villa, 1835) (=zonatus Germar, 1845) csíkoskecskerágó-ormányos 


\section{Glocianus Reitter, 1916 \\ (= Neoglocianus Dieckmann, 1972)}

Glocianus albovittatus (Germar, 1824) (PodLuss ÁNy 2001b) - fehérsávos máktokormányos - Other names: fehércsíku sarla (I. FRIVALDSZKY 1865); fehérsávos ormányos (DUdich 1950, DUdich \& LoKsA 1969, PoDlussánY 2007)

Glocianus brevicollis (Schultze, 1897) - fehérpikkelyes gömbdedormányos

Glocianus distinctus (C. N. F. Brisout de Barneville, 1870) (= marginatus Paykull, 1792) - pitypang-gömbdedormányos (MER KL \& SzÉNÁsı 2018, MER KL et al. 2019, Podlussány 2007) - Other names: gömbded lóhereormányos (DUdich 1950); hölgymál-gömbdedormányos (PodLussánY 2009, 2014); lóhere-gömbdedormányos (PodLusSÁNY 2007, SZÉNÁsi 2012)

Glocianus fennicus (Faust, 1895) (= sparsutus Schultze, 1899) (PodlussáNY 2001b) - márványos gömbdedormányos (PoDLussány 2014)

Glocianus inhumeralis (Schultze, 1897) - szürke gömbdedormányos

Glocianus lethierryi (C. N. F. Brisout de Barneville, 1866) (= incisus Schultze, 1899) - márványos gömbdedormányos - Other names: Lethierry-gömbormányos (MERKL \& SZÉNÁSI 2018)

Glocianusmaculaalba(Herbst, 1795)(Fig.5)-közönséges máktokormányosOther names: mákbogár (NYÁRY 1877); fehérfoltú orrmányos (NYÁRY 1877); máktokormányos (BALÁs \& SÁRINGER 1984, DUdICH 1950, DUDICH \& LOKSA 1969, MERKL \& Vig 2009, Podlussány 2007); máktokbarkó (ENDRŐdi 1968, JABLONOWSKI 1903, MANNINGER 1960, UBRIZSY \& REICHART 1958); máktokormányos (máktokbarkó, mákbarkó, mákormányos, mákbogár) (GoZMÁNY 1979, SÁRINGER in JERMY \& BALÁZs 1990)

Glocianusmoelleri(C.G. Thomson, 1868) - szürkeszőrügömbdedormányos Other names: Möller-gömbdedormányos (PoDLussáNY 2007)

Glocianuspilosellus (Gyllenhal, 1837) - szörösgömbdedormányos(MER KL \& SZÉNÁsi 2018, PoDLUSSÁNy 2007, 2014, SZÉNÁSI 2012)

Glocianus punctiger (C. R. Sahlberg, 1835) - ékeltfarú gömbdedormányos (MER KL \& SZÉNÁSI 2018, PODLUSSÁNY 2007, SZÉNÁsI 2012)

Hadroplontus C. G. Thomson, 1859

Hadroplontus litura (Fabricius, 1775) (Podlussány 1999) - pompás aszatormányos (PoDLUSSÁNY 2009, SzÉNÁsi 2012)

Hadroplontus trimaculatus (Fabricius, 1775) - háromfoltos aszatormányos (Podlussány 2007, 2009, SZÉNÁsi 2012, SzÉNÁsi et al. 2016) - Other names: aszatormányos (Dudich 1950, Dudich \& LoKsa 1969, JolsvaY et al. 1977) 
Micrelus C. G. Thomson, 1859

Micrelus ericae (Gyllenhal, 1813) - sörtés csarabormányos - Other names: csarabormányos (PodLussánY 2007)

\section{Microplontus Wagner, 1944}

Microplontus campestris (Gyllenhal, 1837) - réti margarétaormányos Other names: réti margitvirág-ormányos (PoDLUSSÁNY 2007, 2009)

Microplontus edentulus (Schultze, 1897) - homályos ebszékfüormányos (PodLUSSÁNY 2007)

Microplontus millefolii (Schultze, 1897) (PodLussány 2001b) - nyíljegyesmargarétaormányos

Microplontus molitor (Gyllenhal, 1837) - pipitérormányos - Other names: pipitérszépe-ormányos (PoDlussÁNY 2007)

Microplontus rugulosus (Herbst, 1795) (= chrysanthemi Gyllenhal, 1827; melanostigma Marsham, 1802) - feketeüröm-ormányos (PoDLUSSÁNY 2007,2009, SZÉNÁsI 2012) - Other names: ebszékfü-ormányos (PoDLUSSÁNY 2010)

Microplontus triangulum (Boheman, 1845) - rajzos cickafarkormányos (Podlussány 2007, Merkl et al.2019)

\section{Mogulones Reitter, 1916}

Mogulones abbreviatulus (Fabricius, 1792) - nagy nadálytő-tarkaormányos Other names: közép nadálytőormányos (DuDICH 1950); nadálytőzsuzsok (Gozm ÁNY 1979); óvatos nadálytőormányos (PoDlussá Y 2007, SZÉNÁsi 2012, SZÉNÁsI et al. 2016); közepes nadálytő-ormányos (PoDLussáNY 2009)

Mogulones albolineatus (J. Frivaldszky, 1878) (KRÁt KÝ \& SZYPU€A 2018) sávos tarkaormányos

Mogulones albosignatus (Gyllenhal, 1837) - gyöngyköles-tarkaormányos (MER KL \& SZÉNÁsi 2018, PodLUSSÁNy 2007, SZÉNÁsi 2012)

Mogulones amplipennis (Schultze, 1897) - kisfogú nadálytő-tarkaormányos Other names: kisfogú nadálytőormányos (PoDLussáNY 2007)

Mogulones andreae (Germar, 1824) (= ornatus Gyllenhal, 1837) - szeplőlaputarkaormányos (PoDlussáNy 2007)

Mogulones angulicollis (Schultze, 1897) - laposgalléros tarkaormányos

Mogulones aratridens (Schultze, 1897) - sötét tarkaormányos

Mogulones asperifoliarum (Gyllenhal, 1813) - élénkfoltos tarkaormányos (PodLUSSÁNY 2007, 2009, 2010, SZÉNÁsi 2012) - Other names: borágóormányos (GoZMÁny 1979)

Mogulones aubei (Boheman, 1845) - fekete ebnyelvüfü-tarkaormányos 
Mogulones austriacus (C. N. F. Brisout de Barneville, 1869) - osztrák tarkaormányos (PodLUSSÁNY 2007, SZÉNÁsı 2012)

Mogulones borraginis (Fabricius, 1792) - szürke ebnyelvüfü-tarkaormányos (PodLUSSÁNY 2014)

Mogulones crucifer (Pallas, 1771) (= cruciger Herbst, 1784) - keresztes tarkaormányos (MERKL \& SZÉNÁsI 2018) - Other names: ebnyelvüfü-ormányos (GoZmánY 1979); takaros ebnyelvüfü-ormányos (PoDLUSSÁNY 2007, SZÉNÁsI 2012, SZÉNÁsI et al. 2016); takaros ebnyelvüfü-tarkaormányos (PoDLUSSÁNY 2014)

Mogulones cynoglossi (Frauenfeld, 1866) (PoDLussá NY 2001b) - közönséges ebnyelvüfü-tarkaormányos (MER KL \& SZÉNÁSI 2018, PODLUSSÁNY 2007, 2014); ebnyelvüfü-tarkaormányos (SzÉNÁsı et al. 2016)

Mogulones diecki (C. N. F. Brisout de Barneville, 1870) (= curvistriatus Schultze, 1897) - barázdáshátú tarkaormányos - Other names: Diecktarkaormányos (PoDLUSSÁNY 2007)

Mogulones dimidiatus (I. FRIVALDSZKY, 1865) - apácavirág-tarkaormányos (PoDLUSSÁNY 2007) - Other names: féligfehér sarla (I. FRIVALDSZKY 1865)

Mogulones euphorbiae (C. N. F. Brisout de Barneville, 1866) - nefelejcstarkaormányos (MERKL \& SzÉNÁsi 2018) - Other names: kutyatejormányos (Dudich 1950); nefelejcsormányos(PodLuss ÁnY2007,2009,2014,SZÉNÁsi2012, SZÉNÁsi et al. 2016)

Mogulones geographicus (Goeze, 1777) - térképes kígyószisz-tarkaormányos (MERKL \& VIG 2009) - Other names: térképes ormányos (DUdich 1950, Dudich \& Loksa 1969, Gozmány 1979, Podlussány 2007, SzÉnÁsi 2012); térképes tarkaormányos (MERKL \& SZÉNÁSI 2018)

Mogulones gibbicollis (Schultze, 1897) - nagyfogú tarkaormányos

Mogulones hungaricus (C. N. F. Brisout de Barneville, 1869) - magyar tarkaormányos

Mogulones javetii (Gerhardt, 1867) (= javeti C. N. F. Brisout de Barneville, 1869) - atracél-tarkaormányos - Other names: atracélormányos (DUDICH 1950, PodLUSSÁNy 2007, SZÉNÁsi 2012)

Mogulones larvatus (Schultze, 1897) - tüdőfü-tarkaormányos (PoDLUssá NY 2007, 2009)

Mogulones pallidicornis (Gougelet et $\mathrm{H}$. Brisout de Barneville, 1860) - barna tarkaormányos (PoDlussáNY 2007)

Mogulones pannonicus (Hajóss, 1928) - pannon tarkaormányos

Mogulones raphani (Fabricius, 1792) (= symphyti Bedel, 1885) (PodlussánY 2001b) - kis nadálytő-tarkaormányos (PoDLUSSÁNY 2009, 2014) - Other names: kis nadálytőormányos (Dudich 1950, Dudich \& LoKsa 1969, PodlussáNY 2007, SZÉNÁsi 2012, SZÉNÁsi et al. 2016); közép nadálytőormányos, kis nadálytőormányos (GozMÁNY 1979)

Mogulonesvenedicus(Weise,1879)-sárgapikkelyestarkaormányos(MERKL \& SZÉNÁsI 2018) - Other names: nefelejcs-tarkaormányos (SZÉNÁsI et al. 2016) 
Mogulonoides Colonnelli, 1986

Mogulonoides radula (Germar, 1824) - pettyes atracél-tarkaormányos Other names: pöttyös atracélormányos (PoDLussánY 2007)

Nedyus Schönherr, 1825 (= Cidnorbinus C. G. Thomson, 1859)

Nedyus quadrimaculatus (Linnaeus, 1758) - közönséges csalánormányos (MERKL \& SZÉNÁsi 2018, MERKL et al. 2019) - Other names: csalánormányos (Dudich 1950, Dudich \& Loksa 1969, Gozmány 1979, Podlussány 2007, 2009, 2010, 2014, SzÉNÁsI et al. 2016)

\section{Oprobinus Reitter, 1916}

Oprobinus consputus (Germar, 1824) - hamvas hagymaormányos (MER KL \& SZÉnÁsi 2018, Merkl et al. 2019, Podlussány 2007, 2009, 2014, SzÉnÁsi 2012, SzÉNÁsi et al. 2016)

Oprohinus suturalis (Fabricius, 1775) - csíkos hagymaormányos (PoDLUSSÁNY2007)-Othernames:hagymaormányos(BALÁs \& SÁRINGER 1984, GOZMÁNY 1979); hagymaormányos (hagymabarkó) (SÁRINGER in JERMY \& BALÁZS 1990)

\section{Parethelcus Dieckmann, 1972}

Parethelcuspollinarius (Forster, 1771) - csalánporzó ormányos (MERKL et al. 2019, PodLussány 2007, 2014, SZÉNÁsi et al. 2016)

\section{Phrydiuchus Gozis, 1885}

Phrydiuchus augusti Colonnelli, 2003 (= speiseri Schultze, 1897) kis zsályaormányos (Podlussány \& KuTASI 2011) - Other names: kis zsázsaormányos (SzÉNÁsI et al. 2016)

Phrydiuchus tau Warner, 1969 - szőrös zsályaormányos

Phrydiuchus topiarius (Germar, 1824) - nagy zsályaormányos (PoDlussánY 2014) - Other names: nagy zsázsaormányos (PoDlussánY 2007, SzÉNÁsI et al. 2016) 
Poophagus Schönherr, 1837

Poophagus hopffgarteni Tournier, 1873 - keskeny kányafüormányos - Other names: Hopffgarten-kányafüormányos (PoDLUSSÁ NY 2007)

Poophagus robustus Faust, 1881 - vaskos kányafüormányos

Poophagus sisymbrii (Fabricius, 1777) - hamvas kányafüormányos (PoDlussánY 2007, SZÉNÁsi 2012) - Other names: kányafüormányos (DUDICH 1950, DUdich \& LoKsa 1969, GoZMÁNy 1979)

Prisistus Reitter, 1916

Prisistus kuntzei (Smreczyński, 1957) (SzÉnÁsi et al. 2019) - pusztai bársonyormányos

Prisistusobsoletus(Germar, 1824) - feketebársonyormányos(SzÉNÁsı2012)Other names: bársonyos ormányos (MERKL \& SzÉNÁsI 2018)

Prisistus suturalba (Schultze, 1903) - fehérvarratú bársonyormányos (PoDlussánY 2007); fehérvarratú ormányos (MER KL \& SZÉNÁsI 2018)

Pseudocoeliodes Hoffmann, 1957

Pseudocoeliodes rubricus (Gyllenhal, 1837) - tarka gömböcormányos

Ranunculiphilus Dieckmann, 1970

Austroceutorhynchus Korotyaev, 1980

Ranunculiphilus (Austroceutorhynchus) italicus (C. N. F. Brisout de Barneville, 1869) - egyenesvállú ormányos

Ranunculiphilus Dieckmann, 1970

Ranunculiphilus (Ranunculiphilus) faeculentus (Gyllenhal, 1837) szarkalábormányos (MER KL \& SZÉNÁsı 2018, PODLUSSÁNY 2007, SZÉNÁsI 2012)

Sirocalodes Voss, 1958

Sirocalodes depressicollis (Gyllenhal, 1813) (= nigrinus Marsham, 1802) nyúltcsápú ormányos (PoDLUSSÁNY 2007, SZÉNÁsi 2012)

Sirocalodes quercicola (Paykull, 1792) - füstikeormányos 
Stenocarus C. G. Thomson, 1859

Stenocarus cardui (Herbst, 1784) - pipacsgyökér-ormányos (DUDICH 1950, Dudich \& LoKsa 1969, MERKL \& SzÉnÁsi 2018, MERKL et al. 2019, Podlussány 2007, SZÉnÁsi 2012)

Stenocarus ruficornis (Stephens, 1831) (= fuliginosus Marsham, 1802) mákgyökérormányos (DUDICH 1950, MERKL 2018, MERKL \& SZÉNÁsi 2018, PoDLUSSÁNY 2007, 2014, SZÉNÁsI 2012) - Other names: fekete mákgyökérbarkó (MANNINGER 1960); fekete mákgyökérormányos (BALÁs \& SÁRINGER 1984, KASZAB 1969); fekete mák-gyökérormányos (mákgyökérbarkó) (SÁRINGER in JERMY \& BALÁZS 1990)

Thamiocolus C. G. Thomson, 1859

Thamiocolus imperialis (Schultze, 1895) (DIECKMANN 1973) - pompás fésüsormányos

Thamiocolus kraatzi (C. N. F. Brisout de Barneville, 1869) - vöröscsápú fésüsormányos

Thamiocolus nubeculosus (Gyllenhal, 1837) (PodLussány 2001b) halványfoltos fésűsormányos - Other names: halvány fésűslábú-ormányos (SZÉNÁsI et al. 2016)

Thamiocolus pubicollis (Gyllenhal, 1837) - hosszúorrú fésűsormányos Other names: pölyhöstorju sarla (I. FRIVALDSZKY 1865); hosszúorrú fésüslábúormányos (MERKL \& SZÉNÁsi 2018, PodLussáNy 2007); bakfüormányos (Podlussány 2009, SZÉNÁsi 2012)

Thamiocolus signatus (Gyllenhal, 1837) - tisztesfü-fésűsormányos - Other names: jelelt sarla (I. FRIVALDSZKY 1865); kisebb tisztesfüormányos (DUDICH 1950); kis tisztesfü-ormányos (PodLUSSÁNY 2007)

Thamiocolus viduatus (Gyllenhal, 1813) - barnás fésüsormányos - Other names: mocsári tisztesfüormányos (PoDlussánY 2007, 2009, SZÉNÁsi 2012); barnás fésűslábú-ormányos (MERKL \& SzÉNÁsI 2018)

Thamiocolus virgatus (Gyllenhal, 1837) - macskahere-fésüsormányos

Trichosirocalus Colonnelli, 1979

(= Ceutorrhynchidius Schultze, 1901)

Trichosirocalus barnevillei (Grenier, 1866) - fehérsoros ormányos

Trichosirocalus campanella (Schultze, 1895) - ötfogú ormányos - Other names: ötfogú szemölcsös-ormányos (PoDLUSSÁNY 2007) 
Trichosirocalus horridus (Panzer, 1801) - bütyköstorú aszatormányos (PodlussáNy 2007, 2009, 2014, SZÉNÁsi 2012) - Other names: kormos bogáncsormányos (GOZMÁNY 1979)

Trichosirocalus rufulus (Dufour, 1851) - vöröses útifüormányos

Trichosirocalus spurnyi (Schultze, 1901) (PodlussánY et al. 2016) háromfogú ormányos

Trichosirocalus thalhammeri (Schultze, 1906) - szikiútifü-ormányos

Trichosirocalus troglodytes (Fabricius, 1787) - kis útifüormányos (DUDICH 1950, GoZMÁNY 1979, MER KL \& SZÉnÁsi 2018, MER Kl et al.2019, PodLUSSÁNY 2007, 2010, 2014, SZÉNÁsI 2012, SZÉNÁSI et al. 2016)

\section{Zacladus Reitter, 1913}

Zacladus asperatus (Gyllenhal, 1837) - galléros gólyaorrormányos

Zacladus exiguus (Olivier, 1807) - ráspolyos gólyaorrormányos (Podlussány 2007, SZÉNÁsi et al. 2016)

Zacladus geranii (Paykull, 1800) (= affinis Paykull, 1792) - közönséges gólyaorrormányos (MER KL et al. 2019, PodLUSSÁNY 2007, 2009, 2010) - Other names: geránium-ormányos (GoZMÁNY 1979)

Cnemogonini Colonnelli, 1979

Auleutes Dietz, 1896

Auleutes epilobii (Paykull, 1800) - gömbölyü füzikeormányos - Other names: füzikeormányos (SZÉNÁsı 2012)

Hypurini Schultze, 1902

Aphytobius H. Wagner, 1937

Aphytobius veronicae (J. Frivaldszky, 1884) (KRÁTKÝ 2015) - mécsvirágszöcskeormányos - Other names: vastagcombú veronikaormányos (SzÉNÁsI 2012)

\section{Hypurus Rey, 1882}

Hypurus bertrandi (Perris, 1852) (SZÉNÁsI 2014) - porcsinkeserüfüormányos 
Mononychini LeConte, 1876

Mononychus Germar, 1824

Mononychus punctumalbum (Herbst, 1784) - egykarmú nősziromormányos (Merkl \& Vig 2009, Merkl et al. 2019, Podlussány 2009) - Other names: egykarmú ormányos (DUDICH 1950, DUdich \& LoKSA 1969, GozmánY 1979, HARmos et al.2002, MER KL \& SZÉNÁsi 2018, PodLusSÁNy 2007, SZÉNÁsi 2012, SZÉNÁsi et al. 2016)

\section{Phytobiini Gistel, 1848}

Eubrychius C. G. Thomson, 1859

Eubrychius velutus (Beck, 1817) - úszóhínár-ormányos (DuDICH 1950, PODLUSSÁNY 2007) - Other names: pikkelyes süllőhínár-ormányos (MERKL \& VIG 2009)

\section{Marmaropus Schönherr, 1837}

Marmaropus besseri Gyllenhal, 1837 - Besser-sóskaormányos (PoDlussánY 2007, SZÉNÁsI 2012)

\section{Neophytobius H. Wagner, 1936}

Neophytobius granatus (Gyllenhal, 1835) - márványos keserüfü-ormányos

Neophytobius muricatus (C. N. F. Brisout de Barneville, 1867) - tüskéshátú keserüfü-ormányos

Neophytobius quadrinodosus (Gyllenhal, 1813) - vidrakeserüfü-ormányos (Podlussány 2007, SZÉNÁsi 2012, SZÉNÁsi et al. 2016)

Pelenomus C. G. Thomson, 1859

Pelenomus canaliculatus (Fåhraeus, 1843) - árkostorú keserüfü-ormányos (MER KL \& SZÉNÁsi 2018, Podlussány 2007, SZÉNÁsi 2012)

Pelenomus commari (Panzer, 1795) (= comari Herbst, 1795) - közönséges keserüfü-ormányos (MERKL \& SzÉNÁsI 2018) - Other names: tőzegeperormányos (Podlussány 2007, 2009, SZÉNÁsi et al. 2016) 
Pelenomus quadricorniger (Colonnelli, 1986) (= quadricornis Gyllenhal, 1813) - fogaskarmú keserüfü-ormányos

Pelenomus quadrituberculatus (Fabricius, 1787) - kis keserüfü-ormányos (Dudich 1950, Gozmány 1979, Podlussány 2007, SzÉnÁsi 2012)

Pelenomus velaris (Gyllenhal, 1827) - aranyos keserüfü-ormányos (SzÉNÁsI et al. 2016)

Pelenomus waltoni (Boheman, 1843) - hosszúorrú keserüfüormányos (PoDlussáNy 2007) - Other names: Walton-keserüfüormányos (SzÉNÁsI et al. 2016)

\author{
Phytobius Schönherr, 1833 \\ (= Litodactylus L. Redtenbacher, 1849)
}

Phytobius leucogaster (Marsham, 1802) - süllőhínár-ormányos (MERKL \& SZÉNÁsI 2018, SZÉNÁSI 2012, SZÉNÁSI et al. 2016)

\title{
Rbinoncus Schönherr, 1825
}

Rhinoncus albicinctus Gyllenhal, 1837 - fehérfoltos keserüfü-ormányos (MER KL \& SzÉNÁsi 2018, Podlussány 2007, SzÉNÁsi et al. 2016)

Rhinoncus bosnicus Schultze, 1900 - boszniai keserüfü-ormányos

Rhinoncus bruchoides (Herbst, 1784) - mocsári keserüfü-ormányos (Dudich 1950, Merkl \& SZÉNÁsi 2018, Podlussány 2007, SZÉnÁsi 2012, SzÉNÁsi et al. 2016)

Rhinoncus inconspectus (Herbst, 1795) (= gramineus Fabricius, 1792) - nagy keserüfü-ormányos (DUdICH 1950, Dudich \& LoKsA 1969, MER KL \& SZÉNÁsI 2018, PodLussánY 2007, 2010, SZÉNÁsi 2012, SZÉNÁsi et al. 2016)

Rhinoncus leucostigma (Marsham, 1802) (= pericarpius Paykull, 1792) vöröslábú sóskaormányos (MERKL \& SzÉNÁsi 2018, MERKL et al. 2019, PoDlusSÁNY 2007, SZÉNÁsI 2012, SZÉNÁsI et al.2016) - Other names: vöröslábú juhsóskaormányos (PoDLussáNY 2009, 2014); vöröslábú lórumormányos (Podlussány 2010)

Rhinoncus pericarpius (Linnaeus, 1758) (= castor Fabricius, 1792) - réti keserüfü-ormányos (DUdich 1950, Dudich \& LoKsa 1969, Gozmány 1979, HARMOs et al. 2002, MERKL et al. 2019) - Other names: réti juhsóska-ormányos (PODLUSSÁNY 2007, SZÉNÁsI 2012); élénkfoltosjuhsóskaormányos(MER KL 2018, MERKL \& SZÉNÁSI 2018)

Rhinoncus perpendicularis (Reich, 1797) - foltoshátú keserüfü-ormányos

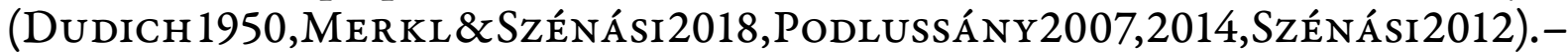
Other names: foltos keserüfü-ormányos (SzÉNÁsI et al. 2016) 
Scleropterini Schultze, 1902

Homorosoma J. Frivaldszky, 1894

Homorosoma validirostre (Gyllenhal, 1837) - fogacskáshátú ormányos

Rutidosoma Stephens, 1831

Rutidosoma Stephens, 1831

Rutidosoma(Rutidosoma)graminosum(Gistel,1857)(=globulusHerbst, 1795)rezgőnyár-gömbölyüormányos (MERKL \& SzÉNÁsi 2018, MERKL et al. 2019, PodlusSÁNY 2007) - Other names: gömbölyü ormányos (PodlussánY 2007, SZÉNÁsI 2012)

Scleropteridius Otto, 1897

Scleropteridius fallax Otto, 1897 - avarlakó madársóska-ormányos - Other names: csalfa ormányos (PoDLussány 2007)

Scleropteridius monticola Otto, 1897 - hegyi madársóska-ormányos - Other names: hegyi ormányos (PoDLUSSÁnY 2007)

Scleropterus Schönherr, 1825

Scleropterus serratus (Germar, 1824) (Fig. 6) - fürészeshátú ormányos

Tapinotus Schönherr, 1826

(= Tapeinotus Schönherr, 1826)

Tapinotus sellatus (Fabricius, 1794) (Fig. 7) - díszes lizinkaormányos (MERKL \& SZÉNÁSI 2018) - Other names: lizinkaormányos (DUDICH 1950, Dudich \& Loksa 1969, Merkl \& Vig 2009, Podlussány 2007, 2009, SZÉNÁSI 2012) 
ConOderitae Schönherr, 1833

Coryssomerini C. G. Thomson, 1859

Coryssomerus Schönherr, 1825

Coryssomerus capucinus (Beck, 1817) - székfü-kancsalormányos (MER KL \& Vig 2009, Podlussány 2007, 2014, SzÉnÁsi 2012) - Other names: székfüormányos (DUdich 1950, GoZMÁNy 1979)

Orobitidita C. G. Thomson, 1859

Orobitidini C. G. Thomson, 1859

Orobitis Germar, 1817

Orobitis cyanea (Linnaeus, 1758) - fémes ibolyaormányos (MERKL \& VIG 2009) - Other names: ibolya-gyökérbarkó (HARmos et al. 2002, HUZIÁN in JeRMy \& Balázs 1990, Podlussány 2007); ibolyaormányos (DUdich 1950, GOZMÁNY 1979)

Orobitis nigrina Reitter, 1885 - fekete ibolyaormányos

Cossoninae Schönherr, 1825

Choerorhinini Folwaczny, 1973

Choerorbinus Fairmaire, 1858

Choerorbinus squalidus Fairmaire, 1858 (MERKL et al. 2010) - sároshátú szúormányos

Cossonini Schönherr, 1825

Cossonus Clairville, 1798

Caenocossonus Voss, 1955

Cossonus (Caenocossonus) cylindricus C. R. Sahlberg, 1835 - hengeres szúormányos (PodlussánY 2007)

Cossonus (Caenocossonus) parallelepipedus (Herbst, 1795) - szögletes szúormányos - Other names: szögletes ormányos (ENDRŐDI 1963, GoZMÁNY 1979) 


\section{Cossonus Clairville, 1798}

Cossonus (Cossonus) linearis (Fabricius, 1775) - korhadék-szúormányos (Merkl \& Vig 2009, Merkl et al. 2019, Podlussány 2007) - Other names: korhadékormányos (DUDICH 1950, DUDICH \& LoKsA 1969, ENDRőDI 1963, GOZMÁNY 1979)

Dryotribini LeConte, 1876

Cotaster Motschulsky, 1851

Cotaster cuneipennis (Aubé, 1850) - szőrös szúormányos (PoDLUssánY 2007)

Onycholipini Wollaston, 1873

Brachytemnus Wollaston, 1873

Brachytemnus porcatus (Germar, 1824) - négyzetestorú szúormányos (MERKL \& SZÉNÁsi 2018) - Other names: négyzetesnyakpajzsú ormányos (KASZAB 1969); négyzetesnyakú ormányos (PoDLussáNy 2007); négyzetesorrú szúormányos (MER KL et al. 2019)

\section{Hexarthrum Wollaston, 1860}

Hexarthrum exiguum (Boheman, 1838) (= culinaris Germar, 1820) változékony bánya-szúormányos (MERKL et al. 2019) - Other names: bányafaormányos (ENDRöDi 1963, JolSVAY et al. 1977, KASZAB 1969); bányafabogár, bányafaormányos (GOZMÁNY 1979); bányafa-szúormányos (MERKL \& VIG 2009)

Pselactus Broun, 1886

(= Codiosoma Bedel, 1885)

Pselactus spadix spadix (Herbst, 1795) - laposszemü szúormányos - Other names: sárgacsápú ormányos (GoZMÁNY 1979) 
Stenoscelis Wollaston, 1861

Stenoscelis Wollaston, 1861

Stenoscelis (Stenoscelis) submuricata (Schönherr, 1832) (SZÉNÁsI 2014) szemölcsös szúormányos

Stereocorynes Wollaston, 1873

Stereocorynes truncorum (Germar, 1824) - sötétbarna szúormányos (ENDRődi 1963, KASZAB 1969, Podlussány 2007)

Rhyncolini Gistel, 1848

Melicius Alonso-Zarazaga, 2002

Melicius cylindrus (Boheman, 1838) (= cylindricus Boheman, 1845) hosszúkás szúormányos (MERKL \& SzÉNÁsi 2018) - Other names: barna szúormányos (DUDICH 1950)

\section{Phloeophagus Schönherr, 1838}

Phloeophagus lignarius (Marsham, 1802) - vaskos szúormányos (MER KL \& VIG 2009) - Other names: lombfa szúormányos (Podlussány 2007)

Phloeophagus thomsoni (Grill, 1898) - barázdás szúormányos

Phloeophagus turbatus Schönherr, 1845 - keskeny szúormányos

Rhyncolus Germar, 1817

Axenomimetes Voss, 1955

Rhyncolus (Axenomimetes) reflexus Boheman, 1838 - szegélyes szúormányos (MERKL \& SZÉNÁSI 2018)

Rhyncolus Germar, 1817

(= Eremotes Wollaston, 1861$)$

Rhyncolus (Rhyncolus) ater ater (Linnaeus, 1758) - fekete szúormányos (Dudich 1950, Merkl \& Vig 2009) 
Rhyncolus (Rhyncolus) elongatus (Gyllenhal, 1827) - nyurga szúormányos (Mer KL 2018, Mer Kl \& SzénÁsi 2018, Mer kl et al. 2019, Podlussány 2007) Rhyncolus (Rhyncolus) punctatulus Boheman, 1838 - pontozott szúormányos (Podlussány 2007, SzÉNÁsi et al. 2016)

Rhyncolus (Rhyncolus) sculpturatus Waltl, 1839 - faragott szúormányos

Curculioninae Latreille, 1802

Acalyptini C. G. Thomson, 1859

Acalyptus Schönherr, 1833

Acalyptus carpini (Fabricius, 1792) - selymes gyertyánormányos (PodlussánY 2007) - Other names: gyertyánormányos (DUDICH 1950, GozMÁNY 1979)

Acalyptus sericeus Gyllenhal, 1835 - selymes füzormányos (MER KL \& SZÉNÁsi 2018, MERKL et al. 2019)

Anoplini Bedel, 1884

Anoplus Germar, 1820

Anoplus plantaris (Naezen, 1794) - nyár-aknázóormányos (PoDlussány 2007) Anoplus roboris Suffrian, 1840 - éger-aknázóormányos (PoDLUssánY 2007) Anoplus setulosus setulosus Kirsch, 1870 - apró aknázóormányos

Anthonomini C. G. Thomson, 1859

Anthonomus Germar, 1817

Anthomorphus Weise, 1883

Anthonomus (Anthomorphus) phyllocola (Herbst, 1795) (= varians Paykull, 1792) - fenyőbarkarontó ormányos (MERKL et al. 2019, PODLUSSÁNY 2007) Other names: bimbórágó ormányos (ENDRöDi 1971, GoZMÁNY 1979, KASZAB 1969, Тóтн 1999)

Anthonomus (Anthomorphus) pinivorax Silfverberg, 1977 (= pubescens Paykull, 1792) - fenyőhajtásrontó ormányos 
Anthonomidius Reitter, 1915

Anthonomus (Anthonomidius) rubripes Gyllenhal, 1835 - pimpóbimbólikasztó (Merkl 2018, Merkl \& SZÉnÁsi 2018, PodlusSány 2007)

\section{Anthonomus Germar, 1817}

Anthonomus (Anthonomus) bituberculatus C. G. Thomson, 1868 - zelnicerügylikasztó (PoDLUssánY 2007, 2014)

Anthonomus(Anthonomus) chevrolatichevrolatiDesbrochersdes Loges, 1868 cseregalagonya-rügylikasztó - Other names: Chevrolat-rügylikasztó (PODLUSSÁNy 2007)

Anthonomus (Anthonomus) conspersus Desbrochers des Loges, 1868 madárberkenye-bimbólikasztó

Anthonomus (Anthonomus) incurvus (Panzer, 1795) (= humeralis Panzer, 1795) - törökmeggy-bimbólikasztó (MERKL \& SzÉnÁsi 2018, PodlussánY 2007) - Other names: cseresznyelikasztó (GozMÁNY 1979)

Anthonomus (Anthonomus) kirschi Desbrochers des Loges, 1868 - fogatlan rügylikasztó - Other names: Kirsch-rügylikasztó (MERKL \& SZÉNÁsi 2018, PODLUSSÁNY 2007)

Anthonomus (Anthonomus) pedicularius (Linnaeus, 1758) - galagonyabimbólikasztó (MER KL \& SzÉNÁsi 2018, MER KL et al. 2019, PODLUSSÁNY 2007, 2009, 2010, 2014, SZÉNÁsi 2012, SZÉNÁsi et al. 2016) - Other names: galagonyaormányos (DUDICH 1950, GozmÁNY 1979)

Anthonomus (Anthonomus) pomorum (Linnaeus, 1758) - almabimbólikasztó (Merke \& SzénÁsi 2018, Merkl \& Vig 2009, Merkl et al. 2019, Podlussány 2007, 2009, SzÉnÁsi 2012) - Other names: alma hópajzs (Divald \& WAGNER 1868); almavirág ormányos (GoZMÁNY 1979, NYÁRY 1877); almavirág ormányos, bimbólyukasztó almaormányos, bimbólikasztó, bimbólikasztó bogár (GoZMÁNY 1979); alma rügyfúróbogár (BALÁs \& SÁRINGER 1984); almarügyfúró bogár (PÁszTor 1901); bimbólyukasztó almaormányos (DUDICH 1950); bimbólyukasztó ormányos (DUDICH \& LoKsA 1969); bimbólikasztó (ENDRÖDI 1971, KASZAB 1969); bombólikasztó (almaormányos, bimbólyukasztó almaormányos) (Jolsvay et al. 1977); bimbólikasztó-ormányos (BALÁs \& SÁRINGER 1984); bimbólikasztó-bogár (almavirág-ormányos, virágrágó almaormányos, égető ormányos, almarügyfúró bogár, bimbólyukasztó ormányos) (REICHART in JERMY \& BALÁzs 1990); bimbólikasztóbogár(JABLONOWSKI 1912, 1917, KADOCSA 1942, REICHART 1951, UBRIZSY \& REICHART 1958, TóTH 1999); almabimbólyukasztó ormányos (GoZMÁNY 1979); almabimbó-lyukasztó (GoZMÁNY 1979)

Anthonomus (Anthonomus) pyri Gyllenhal, 1835 (= cinctus L. Redtenbacher, 1858; = piri Kollar, 1837; = pyri Boheman, 1843) - pompás rügylikasztó 
(MERKL \& SZÉNÁsi 2018, MERKL et al. 2019) - Other names: körtefúró bogár (PÁszToR 1901); rügyfúró körteormányos (DUDICH 1950); körterügylikasztó bogár (UBRIZSY \& REICHART 1958); körterügyfúró ormányos, rügyfúró körteormányos, körterügylikasztó bogár, rügyfúróbogár, rügyfúróormányos (GOZMÁNY 1979); rügyfúróbogár (ENDRŐDI 1971, KASZAB 1969); rügyfúróormányos (BALÁs \& SÁRINGER 1984); rügylikasztó-bogár (körtefúró bogár, rügyfúró bogár, körterügyfúró, rügyfúróormányos, körte-rügylikasztó bogár, körtefúró bogár) (REICHART in JERMY \& BALÁZS 1990); rügylikasztó bogár (TóTH 1999); elegáns rügylikasztó (PodLussáNY 2007)

Anthonomus (Anthonomus) rubi (Herbst, 1795) - szamóca-bimbólikasztó (BALÁs \& SÁringer 1984, GoZMÁNy 1979, MERKL \& SZÉNÁsi 2018, MERKL et al. 2019, SzÉNÁsi et al. 2016, UbRizsy \& REICHART 1958) - Other names: málna bimbólikasztó bogár (KADOcsA 1923); málna bimbólikasztó bogár, málnaormányos, (GoZMÁNY 1979); málnaormányos (DUDICH 1950); málnabimbólikasztó (szamóca-bimbólikasztó bogár, málnaormányos, virágrágó málnaormányos) (REICHART in JERMY \& BALÁzs 1990); málna-bimbólikasztó (HARMos et al. 2002, Tót 1999)

Anthonomus (Anthonomus) rufus Gyllenhal, 1835 (= pruni Desbrochers des Loges, 1868) - kökény-bimbólikasztó (PoDLUSSÁN Y 2007, SZÉNÁsı 2012)

Anthonomus (Anthonomus) sorbi Germar, 1821 - berkenye-bimbólikasztó

Anthonomus (Anthonomus) ulmi (DeGeer, 1775) (= inversus Bedel, 1884) szilfa-bimbólikasztó (MERKL et al. 2019) - Other names: szil-bimbólikasztó (Podlussány 2007, SzÉNÁsi 2012, SzÉnÁsi et al. 2016)

Anthonomus (Anthonomus) undulatus Gyllenhal, 1835 - hullámos bimbólikasztó

Furcipus Desbrochers des Loges, 1868

(= Furcipes Bedel, 1884)

Anthonomus (Furcipus) rectirostris (Linnaeus, 1758) - meggymaglikasztó (Merkl \& Vig 2009, Merkl et al. 2019, Podlussány 2009) - Other names: cseresznyemag fúró (DUDICH \& LoKSA 1969, GozMÁNY 1979); cseresznyemagfú ró ormányos (BALÁs \& SÁRINGER 1984); meggyfúró ormányos (DUDICH 1950, EndRödi 1971, Gozmány 1979, Podlussány 2007, Ubrizsy \& REICHART 1958)

\section{Brachonyx Schönherr, 1825}

Brachonyx pineti (Paykull, 1792) - fenyőtűrágó ormányos (ENDRőDI 1971, Gozmány 1979, Kaszab 1969, Merkl \& SzénÁsi 2018, Merkl et al. 2019, PODLUSSÁNY 2007) 


\section{Bradybatus Germar, 1824 \\ Bradybatus Germar, 1824}

Bradybatus (Bradybatus) creutzeri Germar, 1824 - karcsú juharormányos Other names: Creutzer-ormányos (PoDlussáN Y 2007)

Bradybatus(Bradybatus) kellneriBach, 1854(=subfasciatusGerstäcker,1855)közönséges juharormányos (MERKL \& SzÉNÁsi 2018, MERKL et al. 2019) Other names: juharfaormányos (DUdICH 1950, GozmÁNy 1979); Kellnerormányos (PodlussánY 2007); Kellner-juharormányos (PodlussánY 2009, 2014, SzÉNÁsi 2012, SzÉNÁsi et al. 2016)

Bradybatus (Bradybatus) tomentosus Desbrochers des Loges, 1892 - molyhos juharormányos (MERKL \& SZÉNÁsI 2018)

\section{Nothops Marseul, 1868}

Bradybatus (Nothops) elongatulus (Boheman, 1843) - rozsdás juharormányos

Bradybatus (Nothops) fallax Gerstäcker, 1860 - pompás juharormányos (Podlussány 2007, SZÉnÁsi 2012)

\section{Cionini Schönherr, 1825}

Cionellus Reitter, 1904

Cionellus gibbifrons (Kiesenwetter, 1852) - sokpettyes gömbormányos

\section{Cionus Clairville, 1798}

Cionusalauda(Herbst, 1784)-széleshomlokúgömbormányos(DuDICH 1950, Gozmány 1979, PodLussány 2007)

Cionus clairvillei Boheman, 1838 - nyúlánk gömbormányos (MERKL \& SZÉNÁsi 2018, MERKL et al. 2019) - Other names: Clairville-gömbormányos (Podlussány 2007, SZÉNÁsi 2012)

Cionus ganglbaueri Wingelmüller, 1914 - feketés gömbormányos (Podlussány \& KUTAsi 2011) - Other names: Ganglbauer-gömbormányos (Podlussány 2007, SZÉNÁsi 2012)

Cionusgebleri Gyllenhal, 1838 -lilaökörfarkkóró-gömbormányos(MER KL \& SZÉNÁsi 2018) - Other names: Gebler-gömbormányos (PoDLUSSÁNY 2007)

Cionus hortulanus (Geoffroy, 1785) - gyürüslábú gömbormányos (DUDICH 1950, Dudich \& LoKSA 1969, ENDRŐdi 1971, PODLUSSÁNy 2007, SZÉNÁsI 2012) 
Cionus leonhardi Wingelmüller, 1914 - alföldi gömbormányos (MER KL \& SZÉNÁsI 2018) - Other names: Leonhard-gömbormányos (PODLUSSÁNY 2007)

Cionus longicollis montanus Wingelmüller, 1914 - hosszúorrú gömbormányos

Cionus nigritarsis Reitter, 1904 - feketelábú gömbormányos (PoDlussánY 2007, SZÉNÁsi et al. 2016)

Cionus olens (Fabricius, 1792) - szőrös gömbormányos (PoDLussÁ Ny 2007)

Cionus olivieri Rosenschöld, 1838 - nagy ökörfarkkóró-gömbormányos (MER KL \& SZÉNÁsi 2018, MER KL et al.2019, SZÉNÁsI 2012) - Other names: nagy ökörfarkkóróormányos (DUDICH 1950); nagy gömbormányos (PoDLUSSÁNY 2007)

Cionuspulverosuspulverosus Guérin-Méneville, 1838 - barnagömbormányos (PodLUSSÁNY 2007)

Cionusscrophulariae (Linnaeus, 1758) - görvélyfü-gömbormányos (DUDICH 1950, Dudich \& LoKsa 1969, Endrödi 1971, Gozmány 1979, Merkl \& Vig 2009, PodLussány 2007) - Other names: görvélyfü gömbös ormányos (GoZMÁNy 1979)

Cionus thapsus thapsus (Fabricius, 1792) - kis ökörfarkkóró-gömbormányos (Merkl \& Szénási 2018, Merkl \& Vig 2009, Podlussány 2007, 2009, SZÉNÁsI 2012, SZÉNÁsI et al. 2016) - Other names: kis ökörfarkkóróormányos (Dudich 1950, Dudich \& LoKsa 1969, EndRöDi 1971, Gozmány 1979)

Cionus tuberculosus (Scopoli, 1763) - sávosnyakú gömbormányos (DUDICH 1950, Podlussány 2007, 2009, 2010) - Other names: nagy ökörfarkkóróormányos (GozmÁNY 1979)

\section{Cleopus Dejean, 1821}

Cleopus pulchellus (Herbst, 1795) - rövidszőrű csucsorormányos

Cleopussolani(Fabricius, 1792)-hosszúszörücsucsorormányos(MER KL 2018, Merkl et al.2019, Podlussány 2007)

\section{Stereonychus Suffrian, 1854}

Stereonychus fraxini (DeGeer, 1775) - köris-gömbormányos (ENDRőDI 1971, Gozmány 1979, Harmos et al. 2002, KaSZab 1969, Mer KL \& SzÉnÁsi 2018, MERKL et al. 2019, Podlussány 2007, SzÉnÁsi et al. 2016) - Other names: kőrisfa gömbös ormányos (GozMÁNY 1979) 
Curculionini Latreille, 1802

Archarius Gistel, 1856

Archarius Gistel, 1856

(= Balanobius Jekel, 1861)

Archarius (Archarius) crux (Fabricius, 1777) - keresztes gubacszsuzsóka (MERKL \& SzÉNÁsi 2018) - Other names: keresztes zsuzsóka (DUDICH 1950, Dudich \& LoKsA 1969, ENDRődi 1971, Gozmány 1979, MERKL \& Vig 2009, SZÉNÁSi 2012, PoDlussány 2007, Tóth 1999)

Archarius (Archarius) pyrrhoceras (Marsham, 1802) - tölgy-gubacszsuzsóka (MERKL \& SZÉNÁsi 2018, MERKL et al. 2019) - Other names: gubacszsuzsóka (Dudich 1950, Podlussány 2007, 2009, SzÉNÁsi 2012, SzÉNÁsi et al. 2016)

Archarius (Archarius) salicivorus (Paykull, 1792) - füz-gubacszsuzsóka (MERKL \& SZÉNÁsi 2018, MERKL et al. 2019) - Other names: füzfazsuzsóka (Dudich 1950, Dudich \& LoKsa 1969, Endrödi 1971, Gozmány 1979, SZÉNÁsi 2012, Tóth 1999); füzzsuzsóka (PodLussánY 2007)

\section{Curculio Linnaeus, 1758 \\ Curculio Linnaeus, 1758 \\ (= Balaninus Germar, 1817)}

Curculio (Curculio) betulae (Stephens, 1831) (= cerasorum Herbst, 1791) vöröses égerzsuzsóka - Other names: kökényzsuzsok (DuDICH 1950); cseresznyeormányos, kökényzsuzsok (GozmÁNY 1979); égerzsuzsóka (Podlussány 2007)

Curculio (Curculio) elephas (Gyllenhal, 1835) - gesztenyezsuzsóka (MERKL \& SzÉNÁsi 2018, Podlussány 2007) - Other names: elefántzsuzsok (DUDICH 1950, GYöRFI 1957); elefánt makkfúró ormányos, gesztenyeormányos, gesztenyezsuzsok, elefántzsuzsok(GozMÁNY 1979);gesztenyeormányos(BALÁs\& SÁRINGER 1984, ENDRödi 1971, KASZAB 1969, MERKL \& Vig 2009, SzÉnÁSI 2012, Podlussány 2014, Ubrizsy \& Reichart 1958); gesztenyeormányos (gesztenyezsizsik, gesztenyezsuzsok, elefántzsuzsok) (BÜRGÉs in JERMY \& BALÁzs 1990, Tóth 1999)

Curculio(Curculio)glandium Marsham, 1802-tölgymakkzsuzsóka(MERKL \& SZÉnÁsi 2018, MERKL et al. 2019, Podlussány 2007) - Other names: tölgymakkzsuzsok (DUDICH 1950); makkormányos, tölgymakkormányos, tölgymakkzsuzsok, nagy makkfúró ormányos (GozMÁNY 1979); tölgymakkormányos (BÜRGÉs in JER MY \& BALÁZs 1990, ENDRÖDI 1971, HARMOS et al. 2002, KASZAB 1969, Merkl \& Vig 2009, Podlussány 2014, SzÉNÁsi 2012, SzÉNÁsı et al. 2016); tölgymakkormányos (tölgymakk-zsuzsok) (TóTH 1999) 
Curculio (Curculio) nucum Linnaeus, 1758 - mogyorózsuzsóka (MER KL \& SZÉnÁsi 2018, PodlussánY 2007) - Other names: dió eszelénye (ENTZ 1859); közönséges makkony(DIVALD\&WAGNER 1868); mogyoróbogár(GozMÁNY 1979, JABLONOWSKI 1912); mogyorózsuzsok(DU DICH 1950, FöLDI 1801, GYöR FI 1957); mogyoróormányos (BALÁs \& SÁRINGER 1984, ENDRöDI 1971, KASZAB 1969, MER KL \& Vig 2009, PodLussány 2009, SZÉNÁsi 2012, UBRIZsy \& REICHART 1958); mogyoróormányos (mogyorózsuzsok, mogyorózsizsik, mogyorófúró bogár) (BÜRGÉs in JERMY \& BALÁzs 1990, GOZMÁNY 1979, TóTH 1999)

Curculio (Curculio) pellitus (Boheman, 1843) - makklikasztó zsuzsóka (Merkl \& SzÉNÁsi 2018, Podlussány 2007, SzÉNÁsi 2012, SzÉnÁsi et al. 2016)

Curculio (Curculio) propinquus (Desbrochers des Loges, 1868) - halvány zsuzsóka - Other names: esti zsuzsóka (Podlussány 2007, SzÉNÁsi 2012); halovány estizsuzsóka (MERKL 2018)

Curculio (Curculio) rubidus (Gyllenhal, 1835) - nyírfazsuzsóka - Other names: nyírzsuzsóka (PoDlussánY 2007, SzÉNÁsI 2012)

Curculio (Curculio) venosus venosus (Gravenhorst, 1807) - tarajos zsuzsóka (PoDlussánY 2007,SZÉNÁsi 2012) -Other names: tarajoszsuzsok(DUDICH 1950, GOZMÁNY 1979)

Curculio (Curculio) vicetinus Cussigh, 1989 (SLÁma 2015) - juharzsuzsóka

Curculio (Curculio) villosus Fabricius, 1781 - gubacslakó zsuzsóka (Podlussány 2007, MER KL et al. 2019, TóTH 1999) - Other names: gubacsfúró ormányos, gubacslakó zsuzsok (GoZMÁNY 1979); gubacslakó zsuzsok (DUDICH 1950,DUDICH \& LOKSA 1969, ENDRőDI 1971)

\section{Ellescini C. G. Thomson, 1859 \\ Dorytomus Germar, 1817 \\ Dorytomus Germar, 1817}

Dorytomus (Dorytomus) dejeani Faust, 1883 - közönséges hangormányos (MERkL \& SzÉnÁsi 2018, MERkL et al. 2019) - Other names: Dejeanhangormányos (Podlussány 2007, SZÉNÁsi 2012, SZÉNÁsI et al. 2016)

Dorytomus (Dorytomus) dorsalis (Linnaeus, 1758) - foltos hangormányos

Dorytomus (Dorytomus) filirostris (Gyllenhal, 1835) - egyenesorrú hangormányos (PodLUSSÁN Y 2007, SZÉNÁsi 2012, SZÉNÁsi et al. 2016)

Dorytomus (Dorytomus) hirtipennis Bedel, 1884 - szörös hangormányos (MERKL \& SZÉNÁsi 2018, PodlusSÁNy 2007, SZÉNÁsi 2012, SZÉNÁsi et al. 2016)

Dorytomus (Dorytomus) ictor (Herbst, 1795) (=validirostris Gyllenhal, 1835) szegélyes hangormányos (DUDICH 1950, MERKL et al. 2019, PODLUSSÁNy 2007, SZÉNÁsI 2012, SzÉNÁsi et al. 2016) 
Dorytomus (Dorytomus) longimanus(Forster, 1771) - kétalakú hangormányos (Dudich 1950, Dudich \& LoKsa 1969, Endrödi 1971, Gozmány 1979, HARMos et al.2002, MER KL 2018, MER KL \& SZÉNÁsi 2018, MER KL \& VIG 2009, MERKL et al. 2019, PodLUSSÁNy 2007, SZÉNÁsi 2012, SZÉNÁsi et al. 2016)

Dorytomus (Dorytomus) majalis (Paykull, 1792) - füzbarka-hangormányos (GoZMÁNY 1979) - Other names: apró hangormányos (PoDLUSSÁNY 2007)

Dorytomus (Dorytomus) melanophthalmus (Paykull, 1792) - sárgaorrú hangormányos (Podlussány 2007, 2010, SzÉnÁsi 2012, MERkL \& SZÉNÁsi 2018)

Dorytomus (Dorytomus) minutus (Gyllenhal, 1835) - parányi hangormányos (Podlussány 2007, 2014, SzÉNÁsi 2012, SzÉnÁsi et al. 2016, MERKL \& SZÉNÁSI 2018)

Dorytomus (Dorytomus) nebulosus (Gyllenhal, 1835) - fehérnyárhangormányos (MER KL et al. 2019)

Dorytomus (Dorytomus) nordenskioldi Faust, 1883 - jövevény-hangormányos (PoDlussány 2007)

Dorytomus (Dorytomus) occallescens (Gyllenhal, 1835) - szőrpamacsos hangormányos

Dorytomus (Dorytomus) puberulus (Boheman, 1843) - feketenyárhangormányos (MERKL \& SzÉNÁsi 2018, MERKL et al. 2019) - Other names: márványos hangormányos (PodlussánY 2007, SzÉNÁsi 2012, SzÉNÁsi et al. 2016)

Dorytomus (Dorytomus) rubirostris (Gravenhorst, 1807) (= affinis Paykull, 1800) - tavaszi hangormányos (MERKL \& SzÉNÁsI 2018)

Dorytomus (Dorytomus) rufatus rufatus (Bedel, 1888) - rőt hangormányos (Dudich 1950, Podlussány 2007, SzÉnÁsi 2012)

Dorytomus (Dorytomus) salicinus (Gyllenhal, 1827) - keskenytorú hangormányos

Dorytomus (Dorytomus) salicis Walton, 1851 - kerektorú hangormányos Other names: füz-hangormányos (PoDLUSSÁNY 2007)

Dorytomus (Dorytomus) schoenherri schoenherri Faust, 1883 - sertegalléros hangormányos - Other names: Schönherr-hangormányos (PoDLUSSÁNY 2007)

Dorytomus (Dorytomus) suratus (Gyllenhal, 1836) (= flavipes Panzer, 1797) sárgalábú hangormányos (DUdICH 1950, MER KL \& SZÉNÁsi 2018, PoDLUSSÁNY 2007, SZÉNÁSI 2012)

Dorytomus (Dorytomus) taeniatus (Fabricius, 1781) - kecskefüzhangormányos(MER KL\&SZÉNÁsı2018,PoDLUSSÁNY2007,2010,SZÉNÁSI2012)Other names: füzfa-hangormányos (GozmÁNY 1979)

Dorytomus (Dorytomus) tortrix (Linnaeus, 1760) - csupasz hangormányos (PodlussánY 2007) - Other names: nyárfa-hangormányos (GoZMÁNY 1979)

Dorytomus(Dorytomus) tremulae(Fabricius, 1787)-rezgőnyár-hangormányos (Dudich 1950, MER KL \& SZÉnÁsi 2018, MERkl et al. 2019, Podlussány 2007, 
SZÉNÁSI 2012, SZÉNÁsI et al. 2016) - Other names: rezgőnyárfa hangormányos (Dudich 1950, Dudich \& LoKsa 1969, ENDRöDi 1971)

Dorytomus (Dorytomus) villosulus (Gyllenhal, 1835) - sárgás hangormányos (Dudich 1950, Mer KL \& SZÉnÁsi 2018, Mer Kl et al. 2019, Podlussány 2007, SZÉNÁSI 2012, SZÉNÁsI et al. 2016)

\section{Ellescus Dejean, 1821}

(= Elleschus Schönherr, 1838)

Ellescus bipunctatus (Linnaeus, 1758) - kétpettyes füzormányos - Other names: kétpettyes füzormányos (GozMÁNY 1979)

Ellescus infirmus (Herbst, 1795) - tarka füzormányos

Ellescus scanicus (Paykull, 1792) - sápadt nyárormányos (PodlussánY 2007) -Other names: bodzaormányos (Dudich 1950, GozmánY 1979); sápadt nyárfaormányos (MER KL \& SzÉNÁsI 2018, MERKL et al. 2019, SzÉNÁSI et al. 2016)

Mecinini Gistel, 1848

Cleopomiarus Pierce, 1919

(= Miaromimus F. Solari, 1947)

Cleopomiarus distinctus (Boheman, 1845) - kecses harangvirág-ormányos

Cleopomiarus graminis (Gyllenhal, 1813) - szőrös harangvirág-ormányos (Merke \& Vig 2009, Podlussány 2007, 2009, 2014) - Other names: harangvirág-gubacsormányos (GOZMÁNY 1979)

Cleopomiarus micros (Germar, 1821) - közönséges kékcsillagormányos Other names: kékcsillag-ormányos (MER KL \& VIG 2009)

Cleopomiarus persimilis Smreczyński, 1973 - apró kékcsillagormányos VIG 2009)

Cleopomiarusplantarum (Germar, 1824) - varjúköröm-ormányos (MER KL \&

\section{Gymnetron Schönherr, 1825}

Gymnetron aper Desbrochers des Loges, 1892 - apró veronikaormányos (Mer KL 2018, Merkl \& SzÉNÁsi 2018, Merkl et al. 2019, Podlussány 2009, 2010, 2014, SzÉNÁsi et al. 2016) - Other names: apró útilapu-ormányos (PodLussány 2007)

Gymnetron beccabungae (Linnaeus, 1761) - deréce-veronikaormányos - Other names: deréceormányos (DUdich 1950, GoZMÁNY 1979, PodLUSSÁNY 2007) 
Gymnetron erinaceus (Bedel, 1885) (PoDlussáNy et al. 2017) - vöröslábú veronikaormányos

Gymnetron melanarium (Germar, 1821) - kakukkveronika-ormányos (MER KL 2018, MER KL \& SZÉNÁSi 2018, PodLUSSÁNy 2007, 2010, SZÉNÁSI 2012)

Gymnetron rostellum (Herbst, 1795) - piroslábszárú veronikaormányos (MER KL \& SZÉnÁsi 2018, Mer Kl et al. 2019, PodLussány 2007, SZÉNÁsi 2012, SZÉNÁsi et al. 2016)

Gymnetron rotundicolle Gyllenhal, 1838 (Podlussány et al. 2017) fémesszőrű veronikaormányos (MER KL et al. 2019)

Gymnetron stimulosum (Germar, 1821) - feketelábú veronikaormányos (MERKL 2018) - Other names: feketelábú kamilla-ormányos (PoDlussánY 2007, 2009)

Gymnetron tibiellum Desbrochers des Loges, 1900 (CALDARA 2013) merevsertéjü veronikaormányos

Gymnetron veronicae (Germar, 1821) - mocsári veronikaormányos - Other names: veronika-gubacsormányos (GozMÁNY 1979); foltos veronika-ormányos (PodlussáNy 2007); közönséges veronikaormányos (Podlussány 2009); veronika-ormányos (PoDLUSSÁNY 2010)

Gymnetron villosulum Gyllenhal, 1838 - virágrontó veronikaormányos (MER KL \& SZÉNÁSI 2018, PODLUSSÁNY 2007, SZÉNÁSI 2012)

\section{Mecinus Germar, 1821}

Mecinus circulatus (Marsham, 1802) (Podlussány 2001b) - sávos útifüormányos

Mecinus collaris Germar, 1821 - örvös útifüormányos (DUDICH 1950, HARmos et al. 2002, PodLUSSÁNy 2007, 2009, SZÉNÁsi 2012)

Mecinus heydenii Wencker, 1866 - íveltorrú gyújtoványfü-ormányos Other names: Heyden-útifüormányos (Podlussány 2007, SzÉNÁsi et al. 2016)

Mecinus ictericus (Gyllenhal, 1838) - bundás útifüormányos (MERKL \& SZÉnÁsi 2018, MER KL et al. 2019, PODLUSSÁNy 2007, 2014)

Mecinus janthiniformis Toševski et Caldara, 2011 (TošEvski et al. 2011) déli gyújtoványfü-ormányos (MERKL \& SzÉNÁsI 2018)

Mecinus janthinus Germar, 1821 - kék gyújtoványfü-ormányos (MER KL \& SZÉNÁsi 2018, PoDlussány 2007)

Mecinus labilis (Herbst, 1795) - tarka útifüormányos (MERKL 2018, Merkl \& SZÉnÁsi 2018, Merkl et al. 2019, Podlussány 2007, 2009, 2014, SZÉNÁsI 2012)

Mecinus laeviceps laeviceps Tournier, 1873 (TošEvski et al. 2013) - pusztai gyújtoványfü-ormányos 
Mecinuspascuorum (Gyllenhal, 1813)-közönségesútifüormányos(MER KL \& SZÉNÁsi 2018, MER Kl et al. 2019, PODLUSSÁNy 2007, 2009, 2010, 2014, SZÉNÁsI 2012)

Mecinus pirazzolii (Stierlin, 1867) - homoki útifüormányos (MERKL \& SZÉNÁsi 2018, MERKL et al. 2019) - Other names: Pirazzoli-útifüormányos (Podlussány 2007, 2014)

Mecinus plantaginis (Eppelsheim, 1875) - törpe-útifüormányos (Podlussány 2007)

Mecinuspyraster (Herbst, 1795) - szélesnyakú útifüormányos (DuDICH 1950, Merkl \& SZÉnÁsi 2018, Merkl et al. 2019, Podlussány 2007, 2010, 2014, SzÉNÁsi et al. 2016)

\section{Miarus Schönherr, 1826}

Miarus ajugae (Herbst, 1795) - széles harangvirág-ormányos (MERKL \& Vig 2009, PodLussány 2007, SZÉNÁsi 2012) 2007)

Miarus monticola Petri, 1912 - hegyi harangvirág-ormányos (PodLussánY

Miarus simplex F. Solari, 1947 (KRÁtKÝ \& Podlussány 2008) keleti harangvirág-ormányos - Other names: nagy harangvirág-ormányos (Podlussány \& Kutasi 2011)

Rhinusa Stephens, 1829

Rhinusa antirrbini (Paykull, 1800) - fekete gyújtoványfü-ormányos (MER KL \& SZÉNÁsi 2018, MER KL et al. 2019, PodLuSSÁNy 2007, 2009, SZÉNÁsI 2012, SZÉnÁsi et al. 2016) - Other names: gubacsormányos (GoZMÁNY 1979)

Rhinusaasellus (Gravenhorst, 1807) - szőrcsillagosökörfarkkóró-ormányosOther names: szőrcsillagos ormányos (DUDICH 1950, DuDICH \& LoKsa 1969, ENDRÖDI 1971, MER KL \& VIG 2009, PodlusSÁNy 2007, 2014, SZÉnÁsi 2012); szőrcsillagos gyújtoványfü-ormányos (MER KL 2018, MER KL \& SzÉNÁsI 2018)

Rhinusa bipustulata (Rossi, 1792) - vörösfoltos görvélyfüormányos (PoDlussány 2007)

Rhinusa collina (Gyllenhal, 1813) - lapos gyújtoványfü-ormányos - Other names: csinos gyújtoványfü-ormányos (PoDLUSSÁ NY 2007, 2009)

Rhinusa eversmanni (Rosenschoeld, 1838) (SzÉNÁsI 2014) - aprófogú gyújtoványfü-ormányos

Rhinusa florum (Rübsaamen, 1895) (= smreczynskii Fremuth, 1972) kerekded gyújtoványfü-ormányos (MER KL \& SzÉNÁsi 2018, MER KL et al. 2019)

Rhinusa herbarum (H. Brisout de Barneville, 1862) - borzas gyújtoványfüormányos 
Rhinusa linariae (Panzer, 1795) - gubacsképző gyújtoványfü-ormányos (MER KL \& SZÉNÁsI 2018, PODLUSSÁNy 2014, SZÉNÁsI et al. 2016) - Other names: gubacsképző ormányos (DUdich 1950, GozMÁNY 1979, PodLussá NY 2007)

Rhinusa melas (Boheman, 1838) - szürke gyújtoványfü-ormányos (Podlussány 2007)

Rhinusa neta (Germar, 1821) - drapp gyújtoványfü-ormányos (MERKL \& SZÉnÁsi 2018, MER KL \& Vig 2009, PodLusSÁNy 2007, SZÉNÁsi 2012, SZÉNÁSI et al. 2016)

Rhinusa pilosa (Gyllenhal, 1838) (= hispida Brullé, 1832) (SzÉNÁsı 2014) szőrös gyújtoványfü-ormányos - Other names: szőrös gubacsormányos (GozMÁNY 1979)

Rhinusa rara Toševski et Caldara, 2015 (TošEvski et al. 2015) - egyeneshátú gyújtoványfü-ormányos

Rhinusa tetra (Fabricius, 1792) (= thapsicola Germar, 1821) - magtoklakó ökörfarkkóró-ormányos - Other names: magtoklakó ormányos (DUDICH 1950, Dudich \& Loksa 1969, Gozmány 1979, Harmos et al. 2002, Merkl et al. 2019) - Other names: magtoklakó gyujtoványfü-ormányos (MERKL 2018, Merkl \& SzÉnÁsi 2018, Podlussány 2007, 2014, SzÉnÁsi 2012, SzÉnÁsi et al.2016)

Rhamphini Rafinesque, 1815

Isochnus $\mathrm{C}$. G. Thomson, 1859

Isochnus angustifrons (West, 1917) - törpe-bolhaormányos

Isochnus foliorum (O. F. Müller, 1764) (= saliceti Paykull, 1792) - rekettyefüzbolhaormányos

Isochnus sequensi (Stierlin, 1894) (= populi Fabricius, 1792; = populicola Silfverberg, 1977) - nyár-bolhaormányos (Du DICH 1950, Du DICH \& LoKSA 1969, ENDRöDi 1971, Gozmány 1979, MER KL \& SzÉNÁsi 2018, MER KL \& Vig 2009, Podlussány 2007, 2009, 2010, SzÉNÁsi 2012, Tóth 1999)

Orchestes Illiger, 1798

Alyctus C. G. Thomson, 1859

Orchestes (Alyctus) rusci (Herbst, 1795) - erdei bolhaormányos - Other names: nyírfa-bolhaormányos (GozMÁNY 1979)

Orchestes (Alyctus) testaceus (O. F. Müller, 1776) - nyír-bolhaormányos (Dudich 1950,MER KL\&SZÉNÁsI2018, PodLUSSÁNY2007,2009,SZÉNÁSI2012)Other names: éger bolhaormányos (GozMÁNY 1979, TóTH 1999) 


\section{Orchestes Illiger, 1798}

Orchestes (Orchestes) alni (Linnaeus, 1758) (= saltator Geoffroy, 1785) éger-bolhaormányos (DUDICH 1950) - Other names: nyír-bolhaormányos, égerugróbogár (GoZMÁNY 1979); szilfa-bolhaormányos (GoZMÁNY 1979)

Orchestes (Orchestes) betuleti (Panzer, 1795) (= rufus Schrank, 1781) - vörös bolhaormányos (GozMÁNY 1979)

Orchestes (Orchestes) erythropus (Germar, 1821) - mintás bolhaormányos

Orchestes (Orchestes) fagi fagi (Linnaeus, 1758) - bükk-bolhaormányos (Dudich 1950, EndRöDi 1971, Gozmány 1979, Jolsvay et al. 1977, MER KL \& Vig 2009, PodLussánY 2007, Tóth 1999) - Other names: bükk-ugróormányos, bükk-ugróbogár, bükk ugró ormányosa (GozMÁNY 1979)

Orchestes (Orchestes) hortorum (Fabricius, 1792) (= avellanae Donovan, 1797; = signifer Creutzer, 1799) - változékony bolhaormányos - Other names: változatos bolhaormányos (SzÉNÁsı et al. 2016)

Orchestes (Orchestes) hungaricus (Hajóss, 1938) - magyar bolhaormányos (PodlussánY 2007)

Orchestes (Orchestes) jota (Fabricius, 1787) - kormos bolhaormányos (PoDlussánY 2007)

Orchestes (Orchestes) pilosus (Fabricius, 1781) - bronzos bolhaormányos (PoDlussány 2007)

Orchestes (Orchestes) quedenfeldtii Gerhardt, 1865 - szilfa-bolhaormányos Other names: Quedenfeldt-bolhaormányos (PoDlussánY 2007)

Orchestes (Orchestes) quercus (Linnaeus, 1758) - tölgy-bolhaormányos (Dudich 1950, Dudich \& LoKsa 1969, Endrödi 1971, Gozmány 1979, Merkl et al. 2019, Podlussány 2007, Tóth 1999)

Orchestes (Orchestes) sparsus Fåhraeus, 1843 - foltos bolhaormányos (PoDlussánY 2007)

Orchestes (Orchestes) stobieckii (Smreczyński, 1957) - macskamentabolhaormányos

Orchestes (Orchestes) subfasciatus Gyllenhal, 1835 - szalagos bolhaormányos (PodLussány 2007)

\section{Pseudorchestes Bedel, 1894}

Pseudorchestes cinereus (Fåhraeus, 1843) - fésült bolhaormányos (Podlussány 2007, SZÉNÁsi 2012)

Pseudorchestes ermischi (Dieckmann, 1958) - karcsúlábú bolhaormányos Other names: Ermisch-bolhaormányos (MERKL \& SzÉNÁsi 2018, MERKL \& VIG 2009, PodLussány 2007, 2009, SZÉNÁsi 2012)

Pseudorchestes horioni (Dieckmann, 1958) - serteszőrös bolhaormányos Other names: Horion-bolhaormányos (PoDlussánY 2007) 
Pseudorchestes kostali (Dieckmann, 1985) - sziki bolhaormányos - Other names: Kostál-bolhaormányos (SzÉNÁsI et al. 2016)

Pseudorchestes pratensis (Germar, 1821) - imola-bolhaormányos (MER KL \& SZÉNÁsI 2018, PodLussány 2007, 2009, SZÉNÁsi 2012, SzÉNÁsI et al. 2016)

Pseudorchestes smreczynskii (Dieckmann, 1958) - szálkás bolhaormányos

Rhamphus Clairville, 1798

Rhamphus oxyacanthae (Marsham, 1802) - galagonya-bolhaormányos (Merkl \& Vig 2009, Podlussány 2007, 2009, 2010)

Rhamphus pulicarius (Herbst, 1795) - szil-bolhaormányos (DUDICH 1950, Dudich \&LoKsa 1969, Gozmány 1979, HaRmosetal.2002, PodlussánY 2007, 2009, 2010, SZÉNÁsi 2012)

Rhamphus subaeneus Illiger, 1807 - fémfényü bolhaormányos (MER KL et al. 2019, PodLusSÁNy 2007, 2014, SZÉNÁsi 2012)

Rhynchaenus Clairville, 1798

Rhynchaenus Clairville, 1798

Rhynchaenus(Rhynchaenus)xylosteiClairville, 1798 (=lonicerae Herbst, 1795; = lonicerae Fabricius, 1801) - lonc-bolhaormányos

\section{Tachyerges Schönherr, 1825}

Tachyerges decoratus (Germar, 1821) - díszes bolhaormányos (PoDLUssánY 2007, 2009)

Tachyerges pseudostigma (Tempère, 1982) - egyenesorrú bolhaormányos (MERKL et al. 2019, PodLussány 2007)

Tachyerges rufitarsis (Germar, 1821) - keskenyöves bolhaormányos

Tachyerges salicis (Linnaeus, 1758) - kecskefüz-bolhaormányos (MER KL \& VIG 2009) - Other names: füz-bolhaormányos (DUDICH 1950, DUdich \& LOKSA 1969, ENDRöDi 1971, Gozmány 1979, Podlussány 2007)

Tachyergesstigma(Germar,1821)-fehérjegyesbolhaormányos(DUDICH 1950, Gozmány 1979, Mer KL \& SzÉnÁsi 2018, Podlussány 2007) 
Smicronychini Seidlitz, 1891

Smicronyx Schönherr, 1843

Smicronyx Schönherr, 1843

Smicronyx (Smicronyx) coecus (Reich, 1797) - egykarmú arankaormányos (Podlussány 2007) - Other names: arankaormányos (Dudich 1950, GOZMÁNY 1979)

Smicronyx (Smicronyx) jungermanniae (Reich, 1797) - közönséges arankaormányos (Merkl \& SzÉnÁsi 2018, MERkL \& Vig 2009, Podlussány 2007, SZÉNÁSI 2012, SZÉNÁsi et al. 2016); keskeny arankaormányos (MERKL et al. 2019)

Smicronyx (Smicronyx) nebulosus Tournier, 1874 (= rugicollis Rey, 1895) (GYÖRGY \& PodLUSSÁNY 2005) - felemáskarmú arankaormányos (SZÉNÁsI et al. 2016)

Smicronyx (Smicronyx) reichii (Gyllenhal, 1835) - szemölcsös arankaormányos (PoDlussánY 2007) - Other names: szemölcsös ezerjófü-ormányos (Podlussány 2009, 2014, SZÉNÁsi 2012)

Smicronyx (Smicronyx) smreczynskii F. Solari, 1952 - széles arankaormányos (PodlussáNy 2007) - Other names: Smreczynski-arankaormányos (SzÉNÁsI et al. 2016)

Smicronyx (Smicronyx) striatipennis Tournier, 1874 - vöröslábú arankaormányos (PoDLUSSÁNY 2007)

Smicronyx (Smicronyx) swertiae Voss, 1953 (Podlussány 2001b) gyásztárnics-arankaormányos (MERKL et al. 2019) - Other names: tárnicsormányos (PoDlussáNY 2007); gyásztárnicsormányos (MER KL \& SZÉNÁsI 2018)

Smicronyx (Smicronyx) syriacus Faust, 1887 (= brevicornis F. Solari, 1952) szíriai arankaormányos

Storeini Lacordaire, 1863

Pachytychius Jekel, 1861

Pachytychius sparsutus (Olivier, 1807) - szélestorú rekettyeormányos Other names: rekettyeormányos (DUDICH 1950) 
Styphlini Jekel, 1861

Orthochaetes Germar, 1824

Orthochaetes setiger (Beck, 1817) - levélaknázó sertésormányos - Other names: gerebcsinormányos (GoZMÁNY 1979); pikkelyes ormányos (PoDLUSSÁNY 2007)

\section{Pseudostyphlus Tournier, 1874}

Pseudostyphlus pillumus (Gyllenhal, 1835) (= pilumnus Gravenhorst, 1807) kamilla-sertésormányos (PoDLUSSÁNY 2007) - Other names: kamillaormányos (GoZMÁNY 1979)

Tychiini C. G. Thomson, 1859

Lignyodes Dejean, 1835

Lignyodes Dejean, 1835

Lignyodes (Lignyodes) bischoffi (Blatchley, 1916) - amerikai kőrismagormányos - Other names: fehér körismagormányos (PODLUSSÁNY 2007, SZÉNÁSI 2012, SZÉNÁSI et al. 2016)

Lignyodes (Lignyodes) enucleator (Panzer, 1798) - foltos kőrismagormányos MERKL \& SZÉNÁsI 2018, MER KL et al. 2019) - Other names: tölgyvirágormányos (Dudich 1950, Gozmány 1979, Podlussány 2007, SzÉnÁsi 2012)

Lignyodes (Lignyodes) suturatus Fairmaire, 1860 (= muerlei Ferrari, 1866; =uniformisDesbrochersdesLoges, 1894)-barázdáskőrismagormányos(MERKL\& SZÉNÁsi 2018) - Other names: barázdált magormányos (PODLUSSÁNY 2007, SZÉNÁSI 2012)

Sibinia Germar, 1817

Dichotychius Bedel, 1885

Sibinia (Dichotychius) beckeriDesbrochers des Loges, 1873-sóvirágormányos (SZÉNÁsı et al. 2016) - Other names: Becker-ormányos (SZÉNÁsı 2012) 


\section{Sibinia Germar, 1817}

Sibinia (Sibinia) femoralis Germar, 1824 - foltos habszegfüormányos (MER KL et al.2019, PodLUSSÁNY 2007, 2014) - Other names: habszegfüormányos (MERKL \& SZÉNÁSI 2018)

Sibinia (Sibinia) hopffgarteni Tournier, 1874 - egyszínű madárhúrormányos (MER KL \& SZÉNÁSI 2018) - Other names: Hopffgarten-ormányos (PoDlusSÁnY 2007)

Sibinia (Sibinia) pellucens (Scopoli, 1772) - mécsvirágormányos (MER KL \& SZÉnÁsi 2018, MERKL et al. 2019, Podlussány 2007, 2014, SZÉnÁsi 2012, SZÉNÁsi et al. 2016) - Other names: sziléneormányos (DUDICH 1950, DUDICH \& LoKsA 1969, HaRmos et al. 2002); habszekfü-ormányos (ENDRőDI 1971)

Sibinia (Sibinia) phalerata Gyllenhal, 1835 - középfoltos madárhúrormányos (MERKL \& SZÉNÁsI 2018) - Other names: madárhúr-ormányos (PoDlussánY 2007)

Sibinia (Sibinia) primita (Herbst, 1795) (= signata Panzer, 1809) - bársonyos szalmagyopár-ormányos - Other names: bársonyfoltos ormányos (PoDLUSSÁNY 2007)

Sibinia (Sibinia)pyrrhodactyla (Marsham,1802)(=potentillae Germar, 1824)tarkapikkelyü pimpóormányos - Other names: pimpóormányos (ENDRőDI 1971)

Sibinia (Sibinia) subelliptica (Desbrochers des Loges, 1873) (= fugax Fåhraeus, 1843) - barátszegfü-ormányos (MER KL 2018, MER KL \& SzÉNÁsi 2018, MERKL et al. 2019, PodLussány 2007, 2009)

Sibinia (Sibinia) tibialis Gyllenhal, 1835 - szikárhabszegfü-ormányos (MER KL \& SZÉnÁsi 2018, Podlussány 2007)

Sibinia (Sibinia) unicolor Fåhraeus, 1843 - fátyolvirág-ormányos (MER KL \& SZÉnÁsi 2018, MERKL et al. 2019, PodLussÁNy 2007)

Sibinia (Sibinia) variata Gyllenhal, 1835 - tojásdad csibehúrormányos

Sibinia (Sibinia) viscariae (Linnaeus, 1760) (= fugax Germar, 1824) rendezettpikelyủ habszegfüormányos (MERKL 2018, MERKL \& SzÉNÁsi 2018, MER KL et al. 2019) - Other names: csapzott habszekfü-ormányos (PoDLUssánY 2007, 2014, SZÉNÁSI 2012)

Sibinia (Sibinia) vittata Germar, 1824 - csíkos szegfüormányos (MER KL \& SZÉNÁsi 2018, MER KL et al.2019, PODLUSSÁNY 2007) - Other names: fehérsávos szegfü-ormányos (MERKL \& Vig 2009, PODLUSSÁNY 2014)

Tychius Germar, 1817

Tychius Germar, 1817 (=Aoromius Desbrochers des Loges, 1907; = Miccotrogus Schönherr, 1825)

Tychius (Tychius) aureolus Kiesenwetter, 1852 (= femoralis C. N. F. Brisout de Barneville, 1862) - bolyhos tímárormányos (PoDLussány 2007, 2009) Other names: vörös magormányos (MANNINGER in JERMY \& BALÁzs 1990) 
Tychius (Tychius) breviusculus Desbrochers des Loges, 1873 (= haematopus Gyllenhal, 1835) - laposszemü tímárormányos (MERKL \& SzÉNÁsI 2018, Podlussány 2007, SZÉNÁsI 2012)

Tychius (Tychius) caldarai Dieckmann, 1986 - dárdahere-tímárormányos Other names: Caldara-tímárormányos (PoDLussány 2007)

Tychius (Tychius) crassirostris Kirsch, 1871 - levélgubacs-tímárormányos (Merkl \& SzÉnÁsi 2018, Podlussány 2007, 2009) - Other names: levélgubacsormányos (MANNINGER in JERMY \& BALÁzs 1990)

Tychius (Tychius) cuprifer (Panzer, 1799) - rezesbarna tímárormányos (Merkl et al. 2019, Podlussány 2007, 2009, 2010, 2014, SzÉNÁsi 2012, SZÉNÁsi et al. 2016) - Other names: rezesbarna ormányos (DUDICH 1950, DUDICH \& LOKSA 1969, ENDRÖDI 1971)

Tychius(Tychius)flavusBecker, 1864-lucernamag-tímárormányos(MERKL\& SZÉNÁsi 2018, PodLUSSÁNY 2007, SZÉNÁsi et al. 2016) - Other names: lucernamagormányos (ENDRŐdI 1971, HARMOs et al. 2002, MANNINGER 1960, MANNINGER in JERMY \& BALÁzS 1990); sárga lucernamag-ormányos, lucernamag-ormányos (GoZMÁNY 1979)

Tychius (Tychius) junceus (Reich, 1797) - öszi tímárormányos (MERKL \& SZÉnÁsi 2018, Podlussány 2007, 2009, 2014, SZÉnÁsi 2012, SZÉnÁsi et al. 2016) - Other names: őszi ormányos (DUDICH 1950, Dudich \& LoKsA 1969); őszi magormányos (HARMos et al. 2002, MANNINGER in JERMY \& BALÁzs 1990)

Tychius (Tychius) kulzeri Penecke, 1934 - dülledtszemű tímárormányos (MERKL \& SZÉNÁsi 2018) - Other names: Kulzer-ormányos (PodlussánY 2007); Kulzer-tímárormányos (PoDLussáNY 2014)

Tychius (Tychius) lineatulus Stephens, 1831 - sávos tímárormányos

Tychius (Tychius) medicaginis C. N. F. Brisout de Barneville, 1863 fehércsíkos tímárormányos - Other names: lucerna-tímárormányos (MERKL 2018, Mer KL \& SzÉNÁsi 2018, Podlussány 2007, 2009, 2014, SzÉNÁsi 2012); lucernamag-gubacsormányos (HARMOs et al. 2002, MANNINGER in JERMY \& BALÁzS 1990)

Tychius (Tychius) meliloti Stephens, 1831 - nagyfogú tímárormányos (MER KL 2018, MER KL \& SZÉnÁsi 2018, MER Kl et al.2019, PodlussánY 2007)Other names: fogas tímárormányos (Podlussány 2007, 2009, SZÉnÁsi 2012, SZÉNÁsI et al. 2016); somkoró-magormányos (HARMOs et al. 2002)

Tychius (Tychius) parallelus (Panzer, 1794) - seprőzanót-tímárormányos (Podlussány 2007)

Tychius (Tychius) picirostris (Fabricius, 1787) - szurkosorrú tímárormányos (MER KL \&SZÉNÁsi 2018, MER KL etal.2019, PodlusSÁnY 2007,2009,2010,2014, SZÉNÁsi 2012, SZÉNÁsi et al. 2016) - Other names: szurkosorrú ormányos (DUdich 1950, Dudich \& LoKsa 1969, ENDRődi 1971, HARmos et al. 2002, SÁRINGER in JERMY \& BALÁzS 1990)

Tychius (Tychius) polylineatus (Germar, 1824) - lóhere-tímárormányos 
Tychius (Tychius) pumilus C. N. F. Brisout de Barneville, 1863 (= gabrieli Penecke, 1927) - törpe tímárormányos (MER KL et al. 2019, PoDLussánY 2007, SZÉNÁsi et al. 2016)

Tychius (Tychius) pusillus Germar, 1842 - parányi tímárormányos

Tychius (Tychius) quinquepunctatus (Linnaeus, 1758) -borsó-tímárormányos (MER KL 2018, MER KL \& SZÉNÁsi 2018, MER KL \& Vig 2009, MER KL et al. 2019, PodlussánY 2009, 2014) - Other names: ötpettyes ormányos (DUDICH 1950, GOZMÁNY 1979); borsóormányos (BALÁs \& SÁRINGER 1984, ENDRŐdI 1971, Gozmány 1979, Harmos et al. 2002, Jolsvay et al. 1977, KasZab 1969, MANNINGER 1960, Podlussány 2010, ReichaRT 1957, ReICHART in JeR My \& BALÁZs 1990, SZÉNÁsI 2012, SZÉNÁsI et al. 2016)

Tychius (Tychius) rufipennis C. N. F. Brisout de Barneville, 1863 - vörös tímárormányos (PoDLUSSÁNY 2007)

Tychius (Tychius) schneideri (Herbst, 1795) - nyúlszapuka-tímárormányos (MERKL \& SZÉNÁSI 2018) - Other names: Schneider-tímárormányos (PodLussánY 2007)

Tychius (Tychius) sharpi Tournier, 1874 - fogaslábú tímárormányos

Tychius (Tychius) squamulatus Gyllenhal, 1835 (=flavicollis Stephens, 1831) somkóró-tímárormányos (PoDLUSSÁNY 2009) - Other names: somkóróormányos (DUdich 1950, SÁRINGER in JERMY \& BALÁzs); lucernamag-ormányos (= somkóróormányos) (KASZAB 1969); szarvaskerep-ormányos (SZÉNÁsI 2012); szarvaskerep-tímárormányos (PoDLUSSÁNY 2014)

Tychius (Tychius) stephensi Schönherr, 1835 (= tomentosus Herbst, 1795) rétihere-tímárormányos (MERKL 2018, MERKL \& SzÉNÁsi 2018, MERKL et al. 2019) - Other names: szamócaormányos (DUdich 1950, Gozmány 1979); Stephens-tímárormányos (PodlussánY 2007, 2009, 2010, 2014, SzÉNÁsi 2012)

Tychius (Tychius) subsulcatus Tournier, 1874 - karcsú tímárormányos

Tychius (Tychius) tibialis Boheman, 1843 - fekete tímárormányos (Podlussány 2007, 2010, SZÉNÁsi et al. 2016)

Tychius (Tychius) tridentinus Penecke, 1922 (Podlussány 2001b) - hamvas tímárormányos (MER KL \& SZÉNÁsi 2018)

Tychius (Tychius) trivialis Boheman, 1843 (= kiesenwettweri Tournier, 1873)csüdfü-tímárormányos (MER KL et al. 2019, PODLUSSÁNY 2007)

Cyclominae Schönherr, 1826

Hipporhinini Lacordaire, 1863

Gronops Schönherr, 1823

Gronops Schönherr, 1823

Gronops (Gronops) lunatus (Fabricius, 1775) = félholdas rücskösormányos (MERKL \& VIG 2009) 


\section{Dryophthorinae Schönherr, 1825}

Dryophthorini Schönherr, 1825

Dryophthorus Germar, 1824

Dryophthorus corticalis (Paykull, 1792) - közönséges korhadékzsuzsok (MERKL \& Vig 2009) - Other names: erdei korhadékzsuzsok (MERKL \& SZÉNÁsI 2018); korhadékzsuzsok (PoDLUSSÁNY 2007)

Rhynchophorini Schönherr, 1833

Rhynchophorus Herbst, 1795

Rhynchophorus ferrugineus (Olivier, 1791) (TALLósi 2019) - vörös pálmaormányos (TALLÓsı 2019)

Sitophilus Schönherr, 1838

(= Calandra Gistel, 1848)

Sitophilus granarius (Linnaeus, 1758) - gabonazsuzsok (ENDRőDI 1963, Gozmány 1979, Jávor in Jermy \& Balázs 1990, Merkl \& Vig 2009, Podlussány 2007) - Other names: magtári zsuzsok (Dudich 1950); gabonazsizsik (KASZAB 1969, UBRIZsy \& REICHART 1958); búzaféreg, magtári zsuzsok, gabonazsizsik, magtári zsizsik, zsuzsok, orrbogár (GozMÁNY 1979); magtári gabonazsizsik (magtári zsuzsok, fekete gabonaféreg, gabonazsizsik, magtári zsizsik) (JÁvOR in JERMY \& BALÁZs 1990)

Sitophilus oryzae (Linnaeus, 1763) - rizszsuzsok (ENDRŐDI 1963, DUDICH 1950, Gozmány 1979, Jávor in Jermy \& BALÁzs 1990, MERKL \& Vig 2009, Podlussány 2007, SzÉNÁsi 2012) - Other names: rizszsizsik (Gozmány 1979, KASZA B 1969, UBRIZSY \& REICH ART 1958); rizszsizsik (szurokfekete rizspusztító ormányos) (JÁVOR in JERMY \& BALÁzs 1990)

Sitophilus zeamais Motschulsky, 1855 - barnás kukoricazsuzsok (MER KL \& SZÉNÁsi 2018) - Other names: kukoricazsuzsok (GozMÁNY 1979, JÁvor in JERMY \& BALÁzs 1990); kukoricazsizsik (laplatai kukoricazsizsik) (GoZMÁNY 1979, JÁVOR in JERMY \& BALÁzs 1990) 
Sphenophorus Schönherr, 1838

= Calandra Clairville, 1798)

Sphenophorus abbreviatus (Fabricius, 1787) - óriászsuzsok (DUDICH 1950, MER KL \& VIG 2009, SZÉNÁsI 2012)

Sphenophorus piceus (Pallas, 1771) - sziki zsuzsok (MERKL \& VIG 2009, PoDLUSSÁNY 2014) - Other names: széki zsuzsok (DUDICH 1950, SzÉNÁsi 2012)

Sphenophorus striatopunctatus (Goeze, 1777) - mezei zsuzsok (GozmÁNy 1979, Mer KL \& SZÉnÁsi 2018, Mer Kl \& Vig 2009, Podlussány 2009, SZÉnÁsi 2012) Other names: komócsin-ormányos (ENDRöDI 1963, GozMÁNY 1979, KASZAB 1969, MANNINGER 1960, SÁRINGER in JERMy \& BALÁzs, UBRIZSY \& REICHART 1958)

Entiminae Schönherr, 1823

Brachyderini Schönherr, 1826

Brachyderes Schönherr, 1826

Brachyderes incanus (Linnaeus, 1758) - csillámló ormányos (DUDICH 1950, Gozmány 1979, PodLussány 2007)

Strophomorphus Seidlitz, 1867

Strophomorphus porcellus (Schönherr, 1832) - szálkásszőrű ormányos

Strophosoma Billberg, 1820

Neliocarus C. G. Thomson, 1859

Strophosoma (Neliocarus) faber (Herbst, 1784) - homoki mesterormányos (MERKL 2018, MERKL \& SZÉNÁSI 2018) - Other names: mesterormányos (Podlussány 2007, SzÉNÁsi 2012)

Strophosoma Billberg, 1820

Strophosoma (Strophosoma) capitatum (DeGeer, 1775) - hegyesszemü ormányos - Other names: vöröslábú ormányos (GozMÁNY 1979); foltnélküli ormányos (PoDLUSSÁNY 2007)

Strophosoma(Strophosoma)melanogrammummelanogrammum(Forster, 1771)feketejegyes ormányos (DUdich 1950, DUdich \& LoKSA 1969, Gozmány 1979, MERKL et al.2019, PODLUSSÁNY 2007, SÁRINGER in JERMy \& BALÁzs 1990) 
Byrsopagini Lacordaire, 1863

Graptus Schönherr, 1823

(= Alophus Schönherr, 1826)

Graptus kaufmanni kaufmanni (Stierlin, 1884) - barázdástorú nadálytőormányos - Other names: Kaufmann-nadálytőormányos (SzÉNÁsı 2012, SZÉNÁsi et al. 2016)

Graptus triguttatus triguttatus (Fabricius, 1775) - nagy nadálytőormányos (Dudich 1950, Gozmány 1979, Merk K \& Vig 2009, Podlussány 2007, 2009, 2010, 2014, SZÉNÁsI 2012)

Graptus triguttatus vau (Schrank, 1781) - kárpáti nadálytőormányos

Graptus weberi weberi (Penecke, 1901) (YUNAKOv 2013) - fogascombú nadálytőormányos

Tropiphorus Schönherr, 1842

Tropiphorus cucullatus Fauvel, 1888 - simahátú ormányos

Tropiphorus elevatus (Herbst, 1795) (= carinatus O. F. Müller, 1776) bordáshátú ormányos

Tropiphorus micans Boheman, 1842 - medvehagyma-ormányos (PoDLUSSÁNY 2010) - Other names: csillámló fogancs (I. FrIVALDSZKY 1865); medvehagyma-balgabarkó (PoDLUSSÁNY 2007, 2009)

Cneorhinini Lacordaire, 1863

Philopedon Schönherr, 1826

Philopedon plagiatum (Schaller, 1783) - kerekvállú lomhaormányos - Other names: kerekvállú ormányos (GoZMÁNY 1979)

Geonemini Gistel, 1848

Barynotus Germar, 1817

Barynotus obscurus (Fabricius, 1775) - laposszemü busaormányos - Other names: egérszínü ormányos (GozMÁNY 1979); homályormányos (PODLUSSÁNY 2007) 
Myorhinini Marseul, 1863

Apsis Germar, 1820

(= Myorbinus Schönherr, 1826)

Apsis albolineata (Fabricius, 1792) - alföldi rozsormányos (MERKL 2018, MERKL \& SZÉNÁSI 2018) - Other names: vonalozott gömbör (I. FRIVALDSZKY 1865); keleti ormányos (DUDICH 1950); rozs-ormányos (rozsbogár) (SÁRINGER in Jermy \& BALÁzs 1990); rozsormányos (MERKL \& Vig 2009, PodlussánY 2007, SZÉNÁSI 2012)

Omiini Shuckard, 1840

Amicromias Reitter, 1913

Amicromias zellichi (Formánek, 1907) - pusztai szőrösbarkó - Other names: erdélyi gyepormányos (SZÉNÁsı et al. 2016)

Baromiamima Borovec, 2006

Baromiamima concinna (Boheman, 1834) - barna szőrösbarkó

Elytrodon Schönherr, 1826

Elytrodon bidentatus Boheman, 1834 (MERKL et al. 2010) - északi fogasvégü-ormányos (MERKL et al. 2014) - Other names: kéttövisü röpár (I. FRIVALDSZKY 1865)

Omiamima Silfverberg, 1977

Omiamima mollina (Boheman, 1834) - domború szőrösbarkó

Omias Germar, 1817

(= Mylacus Boheman, 1843)

Omias globulus (Boheman, 1842) - szürke gömböcbarkó (MERKL \& SZÉnÁsi 2018, PodlussánY 2007)

Omias inermis (F. Solari, 1926) (= punctatus Angelov, 1973) - hamvas gömböcbarkó 
Omias puberulus Boheman, 1834 (= rotundatus Fabricius, 1792) fémesszőrü gömböcbarkó (MERKL \& SZÉNÁsi 2018, PODLUSSÁNy 2007, 2014, SZÉNÁsi 2012, SZÉNÁsi et al. 2016)

Omias seminulum (Fabricius, 1792) - magalakú gömböcbarkó (MERKL 2018, Merkl \& SzÉnÁsi 2018, Podlussány 2007, 2014, SzÉnÁsi 2012) Other names: apró serge (I. FRIVALDSZKY 1865); magalakú ormányos (DUDICH 1950, Dudich \& LOKSA 1969)

\section{Rhinomias Reitter, 1894}

Rhinomias austriacus Reitter, 1894 - osztrák duzzadtorrúbarkó (Podlussány 2007, 2010)

Rhinomias forticornis (Boheman, 1842) - tövises duzzadtorrúbarkó - Other names: duzzadtorrú ormányos (DUDICH 1950)

Rhinomias maxillosus Petri, 1891 - törpe duzzadtorrúbarkó

Rhinomias viertli (Weise, 1886) - sárga duzzadtorrúbarkó

Otiorhynchini Schönherr, 1826

Cirrorhynchus Apfelbeck, 1898

Cirrorbynchus arrogans (J. Frivaldszky, 1878) - hosszúszőrü gyalogormányos Cirrorbynchus kelecsenyii (J. Frivaldszky, 1892) - szürke gyalogormányos

\section{Dodecastichus Stierlin, 1861}

Dodecastichus geniculatus (Germar, 1817) - ércesszőrü gyalogormányos

Dodecastichus inflatus (Gyllenhal, 1834) - barázdásorrú gyalogormányos (Dudich 1950, Podlussány 2007, SzÉnÁsi et al. 2016)

Dodecastichus mastix (Olivier, 1807) - lécesorrú gyalogormányos (DUDICH 1950, Gozmány 1979, Mer Kl \& SzÉNÁsi 2018, PodLussány 2007, 2009, 2014, SZÉNÁsI et al. 2016) - Other names: barna vincellérbogár (TóTH 1999)

Dodecastichus pulverulentus (Germar, 1824) - poros gyalogormányos Other names: poros poha (I. FRIVALDSZKY 1865)

Otiorbynchus Germar, 1822 Acunotus Reitter, 1912

Otiorhynchus (Acunotus) lutosus Stierlin, 1858 - avarlakó gyalogormányos 
Amosilnus Reitter, 1912

Otiorhynchus (Amosilnus) lavandus Germar, 1824-lucerna-gyalogormányosOther names: mocskos fogorr (I. FRIVALDSZKY 1865)

Arammichnus Gozis, 1882

Otiorhynchus (Arammichnus) hystrix Gyllenhal, 1834 (= mandibularis W. Redtenbacher, 1842) - sörtés gyalogormányos (PODLUSSÁNY 2007,SZÉNÁsı 2012, SZÉNÁsi et al. 2016, MER KL \& SZÉNÁsI 2018) - Other names: szemercsés fogorr (I. FRIVALDSZKY 1865)

Aranibus Reitter, 1912

Otiorbynchus (Aranihus) frescati Boheman, 1842 - vékonycsápú gyalogormányos

\section{Choilisanus Reitter, 1912}

Otiorhynchus (Choilisanus) brunneus Gyllenhal, 1834 (= jacqueti Stierlin, 1901) - barna gyalogormányos (SzÉNÁsi et al. 2016)

Otiorbynchus (Choilisanus) raucus (Fabricius, 1777) - molyhos gyalogormányos (Dudich 1950, Dudich \& LoKsa 1969, Gozmány 1979, MER KL \& SZÉNÁsi 2018, MER KL et al. 2019, PODLUSSÁNY 2007, 2009, 2010, 2014, SZÉNÁsI 2012, SZÉNÁSI et al. 2016)

Otiorhynchus (Choilisanus) velutinus Germar, 1824 - szörös gyalogor mányos (Dudich 1950, Merkl et al. 2019, Podlussány 2007, 2014) - Other names: molyhos fogorr (I. FRIVALDSZKY 1865)

Crataegodes Bialooki, 2015

Otiorhynchus (Crataegodes) crataegi Germar, 1824 - galagonyagyalogormányos

Cryphiphorus Stierlin, 1883

Otiorhynchus (Cryphiphorus) ligustici (Linnaeus, 1758) (= similis F. Solari, 1932) - vincellér-gyalogormányos (Merkl, 2018, MERKL \& SZÉNÁsi 2018) - 
Other names: hamvas vincellérbogár (BALÁs \& SÁRINGER 1984, DUDICH 1950, DUDICH \& LOKSA 1969, ENDRŐDI 1961b, HARMOs et al. 2002, MERKL \& Vig 2009, Podlussány 2007, 2009, 2014, SzÉNÁsi 2012, SzÉnÁsi et al. 2016, Tóтн 1999); ál-vincellérbogár, hamvas vincellérbogár, nagy vincellérbogár, nagy hamvas vincellérbogár, vincellérbogár, szőlőkapás, szürke vastagorrú (GoZMÁNY 1979); vincellérbogár (UBRIZSY \& REICHART 1958, MANNINGER 1960); hamvas vincellérbogár (hamvas bogár, kiscsajló-ollósbogár, nagy hamvas vincellérbogár, nagyvincellérbogár, vincellérbogár, vintzur-szőlőbogár)(MANNINGERinJER MY \& BALÁZS 1990)

\section{Dorymerus Seidlitz, 1890}

Otiorhynchus (Dorymerus) sulcatus (Fabricius, 1775) - szálkás gyalogormányos (PoDlussáNY 2007) - Other names: barázdás gyalogormányos, barázdáshátú vincellérbogár (GOZMÁNY 1979); barázdáshátú vincellérbogár (BALÁs \& SÁRINGER 1984, MERKL \& TUSNÁDI 1985); barázdáshátú vincellér, barázdáshátú vincellérbogár, gyökérormányos (SÁRINGER in JERMY \& BALÁzS 1990)

\section{Duphanastus Reitter, 1914}

Otiorhynchus (Duphanastus) apfelbecki Stierlin, 1887 (SzÉNÁsı et al. 2019) barnapikkelyes gyalogormányos

\section{Elechranus Reitter, 1912}

Otiorhynchus (Elechranus) roubali Penecke, 1931 - karsztvidéki gyalogormányos - Other names: Roubal-gyalogormányos (MER KL \& VIG 2009)

\section{Lolatismus Reitter, 1912}

Otiorhynchus (Lolatismus) lasius (Germar, 1817) - léceshátú gyalogormányos Otiorhynchus (Lolatismus) porcatus (Herbst, 1795) - tarajos gyalogormányos

Magnanotius Alonso-Zarazaga et Lyal, 2002

Otiorhynchus (Magnanotius) equestris (Richter, 1820) - lomblakó gyalogormányos 
Otiorbynchus (Magnanotius) kollari Gyllenhal, 1834 - sávostorú gyalogormányos

Otiorhynchus (Magnanotius) norici Alonso-Zarazaga, 2013 (= austriacus Fabricius, 1801) - nyugati gyalogormányos

Otiorbynchus (Magnanotius) reichei Stierlin, 1861 - szemölcsöstorú gyalogormányos - Other names: Reiche-gyalogormányos (PoDLussánY \& KUTASI 2011)

Metopiorrhynchus Reitter, 1912

Otiorhynchus (Metopiorrhynchus) labilis Stierlin, 1883 - keskenyormányú gyalogormányos (PODLUSSÁNY 2007)

Otiorbynchus (Metopiorrbynchus) respersus Stierlin, 1861 - erdei gyalogormányos

Mierginus Reitter, 1914

Otiorbynchus (Mierginus) auricapillus (Germar, 1824) - aranypettyes gyalogormányos - Other names: vézna fogorr (I. FRIVALDSZKY 1865)

Nehrodistus Reitter, 1912

Otiorhynchus (Nehrodistus) corruptor corruptor (Host, 1791) - márványos gyalogormányos

Otiorhynchus (Nehrodistus) populeti Boheman, 1842 - nyárligeti gyalogormányos - Other names: nyárligeti fogorr (I. FRIVALDSZKY 1865)

\section{Nibus Reitter, 1912}

Otiorhynchus (Nihus) carinatopunctatus (Retzius, 1783) (= scaber Linnaeus, 1758 sensu Reitter, 1912) - bordás gyalogormányos (DUDICH 1950, DUDICH \& LOKSA 1969)

\section{Otiorhynchus Germar, 1822}

Otiorhynchus (Otiorhynchus) armadillo (Rossi, 1792) - katonás gyalogormányos

Otiorhynchus (Otiorbynchus) bisulcatus (Fabricius, 1781) - egérszürke gyalogormányos (MER KL \& VIG 2009, PodLUSSÁNY 2007) 
Otiorhynchus (Otiorhynchus) cardiniger cardiniger (Host, 1791) - szemölcsös gyalogormányos

Otiorbynchus (Otiorbynchus) coecus coecus Germar, 1824 (= niger Fabricius, 1775) - vöröslábú gyalogormányos (Gozm Á NY 1979, KASZA B 1969, Tó TH 1999) Other names: nagy fekete ormányosbogár (ЕмICH 1899); fekete ormányos, piroslábú vastagorrú ormányos, fekete bogár, vastagorrú bogár, nagy fekete ormányos bogár (GOZMÁNY 1979)

Otiorhynchus (Otiorhynchus) hungaricus hungaricus Germar, 1824 - magyar gyalogormányos (DUdich 1950, DUdich \& LoKSA 1969, PodLussány 2007) Other names: magyarhoni fogorr (I. FRIVALDSZKY 1865)

Otiorbynchus (Otiorbynchus) lirus Schönherr, 1834 (= laevigatus (Fabricius, 1792; cornicinus Stierlin, 1861) - fényes gyalogormányos (DUDICH 1950, GoZmány 1979, PodLussány 2007, 2010)

Otiorhynchus (Otiorbynchus) meridionalis Gyllenhal, 1834(SzÉNÁsı 2016) jövevény gyalogormányos

Otiorhynchus (Otiorhynchus) multipunctatus (Fabricius, 1792) - sokpontos gyalogormányos (KASZA B 1969, PodLuSSÁNY 2007, Tó TH 1999)

Otiorhynchus (Otiorhynchus) pseudonothus Apfelbeck, 1897 (= salicicola Heyden, 1908) (SzÉNÁsI et al. 2019) - aprószemölcsös gyalogormányos

Otiorbynchus (Otiorbynchus) repletus Boheman, 1842 - aranyos gyalogormányos

Otiorhynchus (Otiorhynchus) tenebricosus (Herbst, 1784) (= fuscipes Olivier, 1807) - rozsdáslábú gyalogormányos (DUDICH 1950, GozMÁNY 1979)

Padilehus Reitter, 1912

Otiorhynchus (Padilehus) pinastri (Herbst, 1795) - fenyőrontó gyalogormányos

Paracryphiphorus Magnano, 1998

Otiorhynchus (Paracryphiphorus) catenulatus (Panzer, 1795) (= orbicularis Herbst, 1795) = gyászos gyalogormányos (DUDICH 1950, Du DICH \& LoKsA 1969, PODLUSSÁNY 2007); fekete vincellérbogár (SÁRINGER in JER MY \& BALÁzS 1990)

Pendragon Gozis, 1885

Otiorhynchus (Pendragon) ovatus ovatus (Linnaeus, 1758) - apró gyalogormányos (DUDICH 1950, GozMÁNY 1979, MANNINGER in JERMY \& BALÁZs 1990, MER KL \& SzÉNÁSI 2018, MERKL \& VIG 2009, MERKL et al.2019, 
Podlussány 2007, 2014, SZÉnÁsi 2012, SzÉNÁsi et al. 2016, Tóth 1999) Other names: kis szamócavincellér (apró gyalogormányos) (KASZAB 1969); kis szamócavincellér (BALÁs \& SÁRINGER 1984); szamóca kis ormányosa, kis szamócavincellér, szamócagyökér-ormányos (GozMÁNY 1979); szamócagyökérormányos (KovÁcs 1962); szamócagyökér kis ormányos (KovÁcs 1962, Tóтн 1962); kis szamócavincellér (kis szamócaormányos, szamócagyökér kis ormányos, szamócagyökér-ormányos) (MANNINGER in JERMY \& BALÁzs 1990); kis szamócavincellér (szamócagyökér-ormányos) (Тó TH 1999)

Podoropelmus Reitter, 1912

Otiorhynchus (Podoropelmus) coarctatus Stierlin, 1861 - ráspolyostorú gyalogormányos (PoDLUSSÁNY 2007, SzÉNÁsi 2012)

Otiorbynchus (Podoropelmus) fullo (Schrank, 1781) - aranypikkelyes gyalogormányos (DUDICH 1950, GozMÁNY 1979, KASZA B 1969, MANNINGER in Jermy \& Balázs 1990, Podlussány 2007, 2009, 2014, SzÉnÁsi et al. 2016) - Other names: kalló vincellér (BALÁs \& SÁRINGER 1984); kállóvincellér (GOZMÁNY 1979, KASZAB 1969); kalló vincellérbogár (kalló-vincellér) (MANNINGER in JERMY \& BALÁzs 1990); kalló vincellérbogár (TóTH 1999)

Otiorbynchus (Podoropelmus) juglandis Apfelbeck, 1895 (GYörgY \& PodLussá Y 2005) - érdeshátú gyalogormányos

Otiorhynchus (Podoropelmus) pauxillus Rosenhauer, 1847 (PodlussánY 2001b) - fenyőalja-gyalogormányos (PoDLussány 2007)

Otiorhynchus (Podoropelmus) rotundus Marseul, 1872 (= smreczynskii Cmoluch, 1968) (PodLussáNY et al. 2016) - zömök gyalogormányos

\section{Prilisvanus Reitter, 1912}

Otiorhynchus (Prilisvanus) gemmatus (Scopoli, 1763) - ékköves gyalogormányos (DUDICH 1950, MERKL \& VIG 2009)

Otiorhynchus (Prilisvanus) opulentus Germar, 1836 - ékszer-gyalogormányos - Other names: dús fogorr (I. FRIVALDSZKY 1865)

Provadilus Reitter, 1912

Otiorhynchus (Provadilus) rugifrons (Gyllenhal, 1813) - érdes gyalogormányos (PoDlusSÁNY 2007) - Other names: epergyökér-gyalogormányos (GoZmány 1979) 
Pseudocryphiphorus Magnano, 1998

Otiorhynchus (Pseudocryphiphorus) chrysostictus Gyllenhal, 1834 (= conspersus Herbst, 1795) - hintett gyalogormányos (DUDICH 1950, SzÉNÁSI et al. 2016)

Satnalistus Reitter, 1912

Otiorhynchus (Satnalistus) duinensis Germar, 1824 - kerekpikkelyes gyalogormányos (PoDLUSSÁNY 2007, 2009)

Otiorbynchus (Satnalistus) stenorostris Apfelbeck, 1898 - zártpikkelyes gyalogormányos

Stupamacus Reitter, 1912

Otiorbynchus (Stupamacus) winkleri winkleri F. Solari, 1937 (PodlussánY 2001b) - fényesfekete gyalogormányos

Thalycrynchus Reitter, 1912

Otiorhynchus (Thalycrynchus) perdix (Olivier, 1807) - lapos gyalogormányos (PodLUSSÁNy 2007)

Urorrhynchus Reitter, 1912

Otiorbynchus (Urorrbynchus) sabulosus sabulosus Gyllenhal, 1834 lejtőshátú gyalogormányos

Otiorhynchus (Urorrbynchus) sensitivus (Scopoli, 1763) - csúcsos gyalogormányos (GoZmánY 1979)

\section{Zustalestus Reitter, 1912}

Otiorhynchus (Zustalestus) rugosostriatus (Goeze, 1777) - szamócagyalogormányos (MERkL \& SzÉNÁsi 2018, PodLussány 2007, SZÉnÁsi et al. 2016) - Other names: nagy szamócavincellér (BALÁs \& SÁRINGER 1984, GozmÁNy 1979, KAsZAB 1969, Tóth 1999); szamócagyökér nagy ormányosa (GoZMÁNY 1979, Tó TH 1962); nagy szamócavincellér (szamócagyökér nagy ormányos, nagy szamócavincellérbogár) (MANNINGER in JERMY \& BALÁzS 1990) 
Stomodes Schönherr, 1826

Stomodes gyrosicollis Boheman, 1842 - gyökérlakó ormányos (DUDICH 1950, Podlussány 2007, 2009, 2014, SZÉNÁsi 2012, SZÉNÁsi et al. 2016)

\author{
Peritelini Lacordaire, 1863
}

Centricnemus Germar, 1827

Centricnemus leucogrammus (Germar, 1824) - kis kendermagbogár (Dudich 1950, Dudich \& LoKSA 1969, MER KL 2018, MER KL \& SzÉNÁsi 2018, PODLUSSÁNY 2007)

\title{
Peritelus Germar, 1824
}

Peritelus familiaris Boheman, 1834 - nagy kendermagbogár (DuDICH 1950, Győrfi 1957, Gozmány 1979, Merkl \& Vig 2009, Podlussány 2007, 2014, SZÉNÁsI 2012) - Other names: családos végkövély (I. FRIVALDSZKY 1865); kis kendermagbogár, kendermagbogár (GoZMÁNY 1979); kendermagbogár (BALÁs \& SÁringer 1984, BogNár 1952, ENDRődi 1961b, KASZab 1969, MANNINGER 1960, UBRIZSY \& REICHART 1958); kendermagbogár (nagy kendermagbogár, vincellér, kis vincellér) (REICHART in JERMY \& BALÁzs 1990); kendermagbogár (nagy kendermagbogár, kis vincellér) (Tó TH 1999); közönséges kendermagbogár (MER KL 2018, MER KL \& SZÉNÁsi 2018, MER Kl et al. 2019)

$$
\begin{gathered}
\text { Simo Dejean, } 1821 \\
(=\text { Homorhythmus Bedel, 1883) }
\end{gathered}
$$

Simo hirticornis (Herbst, 1795) - keskeny avar-gyalogormányos - Other names: szőröscsápú gyalogormányos (GoZMÁNY 1979)

Simo variegatus (Boheman, 1842) (PodLussáNy 2001b) - vaskos avargyalogormányos - Other names: vaskos mulyaormányos (PoDLUSSÁNY 2007) 
Phyllobiini Schönherr, 1826

Argoptochus Weise, 1883

Argoptochus Weise, 1883

Argoptochus(Argoptochus) bisignatus (Germar, 1824) - kétfoltosárgusbarkóOther names: kétjegyü csürköcz (I. FRIVALDSZKY 1865)

Argoptochus(Argoptochus) quadrisignatus(Bach, 1856)(SzÉNÁsi etal.2019)mintás árgusbarkó

Argoptochus(Argoptochus) vindobonensis(Formánek, 1908)(Oszonics 1997) (= latirostris Penecke, 1928) - bécskörnyéki árgusbarkó - Other names: bécskörnyéki szőrösbarkó (SzÉNÁsı et al. 2016)

Foucartidius Pesarini, 1981

Argoptochus (Foucartidius) subsignatus (Boheman, 1834) - simatorú árgusbarkó

Mylacoptochus Pesarini, 1981

Argoptochus(Mylacoptochus) viridilimbatus(Apfelbeck, 1898)(PodLussán Y 2001b) - szegélyes árgusbarkó (PoDLUSSÁNY 2007)

Mylacoptochus Korotyaev, 1992

Argoptochus (Mylacoptochus) periteloides (Fuss, 1861) - szőrös árgusbarkó

Oedecnemidius K. Daniel, 1903

Oedecnemidius varius (Brullé, 1832) (= pictus Gyllenhal, 1834) - tarka tölgyormányos (MERKL et al.2019, Podlussány 2007)

Phyllobius Germar, 1824

Alsus Motschulsky, 1845

Phyllobius (Alsus) brevis Gyllenhal, 1834 - rövid levélormányos

Phyllobius (Alsus) dispar dispar L. Redtenbacher, 1847 - fémesszőrü levélormányos 


\section{Dieletus Reitter, 1916}

Phyllobius (Dieletus) argentatus argentatus (Linnaeus, 1758) - ezüstös levélormányos (Gozmány 1979, KASZAB 1969, MERKL \& SzÉnÁsi 2018, MER KL \& Vig 2009, Podlussány 2007, 2009, 2010, 2014, SZÉnÁsi 2012, SZÉnÁsi et al. 2016, Тóтн 1999) - Other names: közönséges levélormányos (DUDICH 1950); ezüstös levélbarkó (ezüstzöld levélormányos, ezüstfényes zöldormányos, keskenyhasú zöldormányos) (GoZMÁNY 1979, REICHART in JERMY \& BALÁzS 1990); ezüstös levélbarkó (BALÁs \& SÁRINGER 1984); ezüstös levélbarkó, ezüstzöld levélormányos (Тóтн 1999)

\section{Metaphyllobius Smirnov, 1913}

Phyllobius (Metaphyllobius) glaucus (Scopoli, 1763) (= calcaratus Fabricius, 1792) - éger-levélormányos (PodLussá Ny 2007, 2009, 2010, SzÉNÁsi 2012, Tó TH 1999) - Other names: sarkantyús levélormányos (DUDICH 1950, DUDICH \& LOKSA 1969, GOZMÁNY 1979, SÁRINGER in JERMY \& BALÁzs 1990)

Phyllobius (Metaphyllobius) pilicornis Desbrochers des Loges, 1872 sarkantyús levélormányos

Phyllobius (Metaphyllobius) pomaceus Gyllenhal, 1834 (= urticae DeGeer, 1775) - csalán-levélormányos (PodLussány 2007, 2009, 2014) - Other names: éger-levélormányos (GoZmáNY 1979, KASZAB 1969); éger-levélbarkó (HARMOS et al. 2002)

\section{Nemoicus Dillwyn, 1829}

Phyllobius (Nemoicus) oblongus (Linnaeus, 1758) - közönséges levélormányos (Dudich 1950, Dudich \& LoKsa 1969, Mer KL \& SzÉNÁsi 2018, MER KL \& VIG 2009, Merkl et al. 2019, Podlussány 2007, 2009, 2010, 2014, SzÉnÁsi 2012, SzÉNÁsi et al. 2016, Tóth 1999) - Other names: közönséges lombormányos (ENDRöDI 1961b); közönséges levélbarkó (barna levélbarkó, tölgylevélbarkó hosszúkás levélrágó) (REICHART in JERMY \& BALÁzs 1990); közönséges levélbarkó (BALÁs \& SÁRINGER 1984, HARMOS et al. 2002); barna levélbarkó, tölgylevélbarkó, hosszúkás levélrágó (TóTH 1999)

\section{Parnemoicus Schilsky, 1911}

Phyllobius(Parnemoicus) roboretanusGredler, 1882(=parvulusOlivier,1807)bársonyos levélormányos 
Phyllobius(Parnemoicus) viridicollis(Fabricius, 1792) - zömöklevélormányos (MERKL \& SZÉNÁsI 2018)

\section{Phyllobius Germar, 1824}

Phyllobius (Phyllobius) arborator (Herbst, 1797) - aranyos levélormányos (Dudich 1950, Dudich \& Loksa 1969, Gozmány 1979, Podlussány 2007)

Phyllobius (Phyllobius) betulinus betulinus (Bechstein et Scharfenberg, 1805) - változékony levélormányos (MER KL et al.2019, PoDLUSSÁNY 2007, 2009, 2014, SZÉNÁsi 2012, SzÉNÁsi et al. 2016) - Other names: aranyszínü levelény (I. FRIVA LDSZKY 1865); nyírfalevélormányos (DUdich 1950, DUDICH \& LoKSA 1969); nyírfa levélbarkó (HA Rmos et al. 2002)

Phyllobius (Phyllobius) pyri (Linnaeus, 1758) (=vespertinus Fabricius, 1792) gyümölcsfa-levélormányos (DUdich 1950, DUDICH \& LoKSA 1969, GozMÁNY 1979, Merkl \& SzénÁsi 2018, Merkl \& Vig 2009, Merkl et al. 2019, PodLUSSÁNy 2007, 2009, 2014, SZÉNÁsi 2012, SzÉNÁsi et al. 2016, Tóth 1999) Other names: gyümölcsfa-levélbarkó (fekete levélormányos, körtelevélbarkó, körtefás levélrágó, sávozott zöldormányos (REICH ART in JER MY \& BALÁzs 1990); gyümölcsfa-levélbarkó (BALÁs \& SÁRINGER 1984, GozMÁNY 1979, HARMOS et al. 2002); pufók levélormányos (MER KL \& SZÉNÁSi 2018, MERKL et al. 2019, PodlussánY 2007, 2014, SZÉNÁsi 2012); pufókfejű levélormányos (SzÉNÁsI et al. 2016)

Phyllobius (Phyllobius) seladonius Brullé, 1832 - kardlábú levélormányos (SzÉNÁsi et al. 2016) - Other names: tompaorrú levélormányos (PoDlussánY 2007, 2009, 2014)

Phyllobius (Phyllobius) thalassinus Gyllenhal, 1834 (= scutellaris L. Redtenbacher, 1849) - tömpeorrú levélormányos - Other names: zöld pázsitfüormányos (PodLussány 2007, SzÉNÁsi 2012, SzÉNÁsi et al. 2016)

\section{Plagius Desbrochers des Loges, 1873}

Phyllobius (Plagius) montanus Miller, 1862 - hegyi levélormányos (Podlussány 2007)

Phyllobius (Plagius) pallidus (Fabricius, 1792) (= incanus Gyllenhal, 1834) tölgy-levélormányos (MERKL et al. 2019, Podlussány 2007, 2014) - Other names: fehérszürke levelény (I. FRIVALDSZKY 1865) 
Pterygorrhynchus Pesarini, 1969

Phyllobius (Pterygorrhynchus) maculicornis (Germar, 1824) - galagonyalevélormányos (Dudich 1950, Dudich \& LoKsa 1969, Gozmány 1979, MERKL et al. 2019, PodLUSSÁNy 2007, 2010, 2014, SZÉnÁSi 2012)

\section{Subphyllobius Schilsky, 1911}

Phyllobius (Subphyllobius) virideaeris virideaeris (Laicharting, 1781) halványzöld levélormányos (MERKL 2018, MERKL \& SZÉNÁsi 2018, MERKL et al. 2019, SzÉNÁsi 2012) - Other names: érczöld levélormányos (DUDICH 1950, GoZmÁnY 1979); halványzöld ormányos (PodLussány 2007, 2010, 2014)

Pseudomyllocerus Desbrochers des Loges, 1872

Pseudomyllocerus invreae F. Solari, 1948 (= cinerascens Fabricius, 1792) szürke levélormányos

Pseudomyllocerus sinuatus (Fabricius, 1801) - szeder-levélormányos (MER KL \& SZÉNÁsi 2018) - Other names: szederormányos (PodlussánY 2007, SZÉNÁsI 2012, SZÉNÁsI et al. 2016)

Polydrusini Schönherr, 1823

Liophloeus Germar, 1817

Liophloeodes Weise, 1894

Liophloeus (Liophloeodes) gibbus Boheman, 1842 - laposorrú barabolyormányos - Other names: domború poha (I. FRIVALDSZKY 1865)

Liophloeus (Liophloeodes) lentus Germar, 1824 - közönséges barabolyormányos (PoDlussány 2007) - Other names: lassú poha (I. FRIVALDSZKY 1865)

Liophloeus (Liophloeodes) liptoviensis Weise, 1894 (PoDluss ÁNY 2001b) liptói barabolyormányos

Liophloeus (Liophloeodes) pupillatus Apfelbeck, 1928 - pupillás barabolyormányos 
Liophloeus Germar, 1817

Liophloeus (Liophloeus) tessulatus (O. F. Müller, 1776) - kockás barabolyormányos (MERKL et al. 2019) - Other names: kockás ormányos (Dudich 1950, Gozmány 1979, Harmos et al. 2002, Podlussány 2009)

\section{Pachyrbinus Schönherr, 1823}

Pachyrbinus Schönherr, 1823 (= Scythropus Schönherr, 1826)

Pachyrhinus (Pachyrbinus) squamulosus (Herbst, 1795) (= mustela Herbst, 1797) - busafejü ormányos

\section{Polydrusus Germar, 1817}

Chlorodrosus K. Daniel et J. Daniel, 1898

Polydrusus (Chlorodrosus) amoenus (Germar, 1824) - hegyi lombormányos (PodLussány 2007)

Conocetus Desbrochers des Loges, 1875

Polydrusus (Conocetus) crinipes Germann, 2018 (SzÉNÁsI 2016) - mediterrán lombormányos (SZÉNÁsı 2018)

Eudipnus C. G. Thomson, 1859

Polydrusus (Eudipnus) formosus (Mayer, 1779) (= sericeus Schaller, 1783; $=$ thalassinus Gyllenhal, 1834)-selymeslombormányos(DUDICH 1950,DUDICH \& Loksa 1969, Gozmány 1979, Merkl \& SzénÁsi 2018, Merkl \& Vig 2009, Merkl et al. 2019, Podlussány 2007, 2009, 2010, 2014, SÁRINGER in Jermy \& BALÁzs 1990, SzÉNÁsi 2012, Tóth 1999)

Polydrusus (Eudipnus) mollis (Strøm, 1768) - termetes lombormányos (Dudich 1950, Dudich \& Loksa 1969, Gozmány 1979, Merkl \& Vig 2009, Merkl et al. 2019, Podlussány 2007, Sáringer in Jermy \& Balázs 1990, Tótн 1999) 
Eurodrusus Korotyaev et Meleshko, 1997

Polydrusus (Eurodrusus) cervinus (Linnaeus, 1758) - aranyporos lombormányos (Dudich 1950, Dudich \& LoKsA 1969, Gozmány 1979, Mer KL \& SZÉnÁsi 2018, MERKL et al. 2019, Podlussány 2007, 2009, SÁringer in JERMY \& BALÁzs 1990, SzÉNÁsi 2012, SZÉNÁsi et al. 2016, TóTH 1999)

Polydrusus (Eurodrusus) confluens Stephens, 1831 - sávos lombormányos (MER KL \& SZÉNÁsi 2018, PODLUSSÁNy 2007, SZÉNÁsi 2012)

Polydrusus (Eurodrusus) pilosus pilosus Gredler, 1866 - szörfoltos lombormányos (MERKL et al. 2019, PODLUSSÁNY 2007, 2010)

\section{Eustolus C. G. Thomson, 1859}

Polydrusus (Eustolus) corruscus Germar, 1824 - kecskefüz-lombormányos (Dudich 1950, Dudich \& Loksa 1969, Merkl \& SzéNÁsi 2018, Merkl et al. 2019, Podlussány 2007, 2010, SZÉNÁsi 2012, SzÉNÁsi et al. 2016)

Polydrusus (Eustolus) flavipes flavipes (DeGeer, 1775) - feketeszőrü lombormányos (SzÉNÁsi et al. 2016) - Other names: sárgalábú lombormányos (GoZMÁNy 1979); éger-lombormányos (PoDLUsSÁNy 2007, SZÉNÁsI 2012)

Polydrusus (Eustolus) impressifrons Gyllenhal, 1834 - selymeszöld lombormányos - Other names: nyárfa-lombormányos (GozMÁNY 1979)

Polydrusus (Eustolus) pterygomalis Boheman, 1840 - púposfejü lombormányos (PODLUSSÁNY 2007, 2009, 2014)

\section{Leucodrusus Stierlin, 1884}

Polydrusus (Leucodrusus) tibialis Gyllenhal, 1834 - gyepi lombormányos (MERKL 2018, MERKL \& SZÉNÁSI 2018) - Other names: vastagcsápú kerepormányos (PODLUSSÁNY 2009, 2014)

\section{Metallites Germar, 1824}

Polydrusus (Metallites) aeratus aeratus Gravenhorst, 1807 (= atomarius Olivier, 1807; = pallidus Gyllenhal, 1834) - fakó lombormányos - Other names: hajtásrágó ormányos (GozMÁNY 1979); fakó fenyőormányos (PoDLUSSÁNY 2007) Polydrusus(Metallites) imparGozis, 1882-fenyves-lombormányos(MER KL \& SZÉNÁsi 2018, MERKL et al. 2019) - Other names: hegyvidéki ormányos (GoZMÁNY 1979); páratlan fenyőormányos (PodLUSSÁNY 2007)

Polydrusus (Metallites) marginatus Stephens, 1831 - szegélyes lombormányos (Podlussány 2007) - Other names: szegélyes ormányos (KASZAB 1969) 
Poecilodrusus Korotyaev et Meleshko, 1997

Polydrusus (Poecilodrusus) viridicinctus Gyllenhal, 1834 - zöldöves lombormányos (Dudich 1950, Dudich \& LoKsA 1969, Podlussány 2007, 2014)

\section{Polydrusus Germar, 1817}

Polydrusus (Polydrusus) fulvicornisfulvicornis (Fabricius, 1792) - göröngyöstorú lombormányos

Polydrusus (Polydrusus) picus picus (Fabricius, 1792) - pikkelyfoltos lombormányos - Other names: foltos lombormányos (DUDICH 1950, DUDICH \& LoKsa 1969, Harmos et al. 2002, Merkl \& SzÉnÁsi 2018, Merkl \& Vig 2009, Merkl et al. 2019, Podlussány 2007, 2009, 2010, 2014, SZÉNÁsi 2012, SZÉNÁsi et al. 2016)

Polydrusus (Polydrusus) sparsus Gyllenhal, 1834 - éger-lombormányos (Podlussány 2007

Polydrusus (Polydrusus) tereticollis (DeGeer, 1775) - szalagos lombormányos (Dudich 1950, Dudich \& Loksa 1969, Gozmány 1979, Merkl 2018, PodLUSSÁny 2007, 2010)

\section{Scythodrusus Korotyaev et Meleshko, 1997}

Polydrusus (Scythodrusus) inustus Germar, 1824 (Podlussány et al. 2016) keleti lombormányos - Other names: szőrös lombormányos (SzÉNÁsI et al. 2016)

\section{Psallidiini Lacordaire, 1863 \\ Mesagroicus Schönherr, 1840}

Mesagroicus obscurus Boheman, 1840 - dülledtszemü libatopormányos Other names: dohánybarkó (HUZIÁN in JERMY \& BALÁzS 1990); libatopormányos (PodlussánY 2007, SZÉNÁSI 2012, SZÉNÁSI et al. 2016); szemölcsöstorú ormányos (PoDlussánY 2014) 


\section{Psallidium Herbst, 1795 \\ Psallidium Herbst, 1795}

Psallidium (Psallidium) maxillosum maxillosum (Fabricius, 1792) - fekete barkó (BALÁs 1963, BALÁs \& SÁRINGER 1984, ENDRŐDI 1960, GOZM ÁNY 1979, HARMos et al. 2002, JolsvaY et al. 1977, MANNINGER 1960, MANNINGER in Jermy \& BAlázs 1990, MERKL et al. 2019, Podlussány 2007, 2014, SZÉnÁsi et al. 2016) - Other names: fekete harapós (GoZMÁNY 1979); fekete répabarkó (Jablonowski 1906, Dudich 1950, Dudich \& LoKsa 1969, Gozmány 1979, UBRIZSY \& REICHART 1958); fekete répabarkó (ENDRÖDI 1960, GoZM ÁNY 1979, Jolsvay et al. 1977, MANNINGER 1960); fekete harapós, fekete répabarkó (MANNINGER in JERMY \& BALÁZS 1990)

Sciaphilini Sharp, 1891

Archeophloeus Iablokoff-Khnzorian, 1959

Archeophloeus inermis (Boheman, 1842) - ál-éjiormányos - Other names: védtelen csülköny (I. FRIVALDSZKY 1865) - Other names: kis éjiormányos (Podlussány 2007, SZÉnÁsi 2012, SZÉNÁsi et al. 2016)

Brachysomus Schönherr, 1823

Brachysomus Schönherr, 1823

Brachysomus (Brachysomus) dispar Penecke, 1910 - barna gyepormányos

Brachysomus (Brachysomus) echinatus (Bonsdorff, 1785) - tüskés gyepormányos (Merkl \& Vig 2009, Merkl et al. 2019, Podlussány 2007, 2010, 2014, SZÉNÁsi 2012) - Other names: sertés gyepormányos (DUDICH 1950); sörtés gyepormányos (KASZAB 1969); kockás ormányos, sörtés gyepormányos, sertés gyepormányos (GoZMÁNY 1979)

Brachysomus (Brachysomus) erinaceus (Fabricius, 1801) (= villosulus Germar, 1824) - ritkaszőrü gyepormányos (PodLUSSÁNY 2007, 2014, SzÉNÁsı 2012)

Brachysomus (Brachysomus) fremuthi Koštál, 1991 - pannon gyepormányos Other names: Fremuth-gyepormányos (SzÉNÁsi et al. 2016)

Brachysomus (Brachysomus) frivaldszkyi (Reitter, 1884) - Frivaldszkygyepormányos

Brachysomus (Brachysomus) hegyessyi Yunakov, 2006 (Yunakov 2006) Hegyessy-gyepormányos (MER KL \& VIG 2009)

Brachysomus (Brachysomus) hirtus (Boheman, 1845) - sörtés gyepormányos (Podlussány 2007, 2009, 2014) 
Brachysomus (Brachysomus) hispidus (L. Redtenbacher, 1849) - dülledtszemü gyepormányos (DUDICH 1950, SZÉNÁsI 2012)

Brachysomus (Brachysomus) kratkyi Białooki, 2007 (BIA£OoKI 2007) mátrai gyepormányos

Brachysomus (Brachysomus) mihoki Penecke, 1914 - bakonyi gyepormányos (Podlussány 2007, Merkl \& Vig 2009, Merkl et al. 2019)

Brachysomus (Brachysomus) setiger (Gyllenhal, 1840) - vastagcsápú gyepormányos (DUdich 1950, PoduUssánY 2007, 2009, 2014)

Brachysomus (Brachysomus) slovacicus Koštál, 1991 (PodlussánY 2001b) szlovák gyepormányos (MER KL \& VIG 2009)

Brachysomus (Brachysomus) subnudus (Seidlitz, 1868) - csupasz gyepormányos (Podlussány \& KUTASI 2011)

\section{Hippomias Yunakov, 2006}

Brachysomus (Hippomias) strawinskii Cmoluch, 1960 (KRÁTKÝ \& Podlussány 2008) - erdei gyepormányos

Brachysomus (Hippomias) tenuicollis Yunakov, 2006 (Yunakov 2006) zempléni gyepormányos (MER KL \& VIG 2009)

\section{Eusomus Germar, 1824}

Eusomus ovulum Germar, 1824 - közönséges cickafarkormányos (MERKL 2018, MERKL \& SZÉNÁsi 2018, MERKL et al. 2019) - Other names: cickafarkormányos (Dudich 1950, DUDICH \& LoKsA 1969, ENDRődi 1960, Gozmány 1979, Harmos et al. 2002, Podlussány 2007, 2009, 2010, 2014, SZÉNÁsI 2012, SZÉNÁsI et al. 2016)

\section{Exomias Bedel, 1883}

Exomias araneiformis araneiformis (Schrank, 1781) - simahátú mohaormányos (PoDLUSSÁNY 2007, 2010)

Exomias formaneki (Fremuth, 1971) - Formánek-mohaormányos

Exomias holosericeus (Fabricius, 18012) (= chevrolati Boheman, 1842) nagyfogú mohaormányos (MERKL \& VIG 2009) - Other names: füormányos (Dudich 1950); Chevrolat-ormányos (Podlussány 2007); Chevrolatmohaormányos (PodLUSSÁNY 2010)

Exomiasinterpositusinterpositus (Roubal, 1920) - sarkantyús mohaormányos

Exomias interpositus siliciensis (Fremuth, 1971) (PodLussáNy 1999) szilicei mohaormányos 
Exomias liptoviensis (Weise, 1894) (Podlussány 2001b) - liptói mohaormányos

Exomias mollicomus (Ahrens, 1812) - bolyhos mohaormányos (MER KL et al. 2019, Podluss ÁnY 2007, 2014, SZÉnÁsi et al. 2016)

Exomias paratenex (Fremuth, 1981) (KRÁTKÝ \& PodLussáNy 2008) hegyi mohaormányos - Other names: parány mohaormányos (PoDlussÁNY \& KUTASI 2011)

Exomias pellucidus pellucidus (Boheman, 1834) - nagy mohaormányos (PoDlussáNY 2007) - Other names: bolyhos mohaormányos [mistake] (SZÉNÁSI et al. 2016)

Exomiasstyriacus (Seidlitz, 1868) - stíriai mohaormányos (PoDlussány 2009)

Exomias tenex (Boheman, 1843) - apró mohaormányos - Other names: ritka mohaormányos (PoDLUSSÁNY 2007)

Foucartia Jacquelin du Val, 1854

(= Parafoucartia F. Solari, 1948)

Foucartia liturata Stierlin, 1884 - pusztai törpebarkó - Other names: parti törpebarkó (SZÉNÁsı 2012) 2007)

Foucartia ptochoides (Bach, 1856) - zöldpikkelyes törpebarkó (PoDLUSSÁNY

Foucartia squamulata (Herbst, 1795) (= burghauseri Reitter, 1905) közönséges törpebarkó - Other names: pikkelyes kerepormányos (DUDICH 1950, Harmos et al. 2002, Podlussány 2007, 2010); zöldpikkelyes törpebarkó (SZÉNÁSI 2012, SZÉNÁsi et al. 2016)

\section{Paophilus Faust, 1891}

Paophilus afflatus (Boheman, 1833) - piroslábú pimpóormányos (Podlussány 2007, SzÉNÁsi 2012)

Paophilus hampei (Seidlitz, 1867) - rezgőpázsit-pimpóormányos (DUDICH 1950, HARmos et al. 2002, SzÉNÁsi et al. 2016)

Sciaphilus Schönherr, 1823

Sciaphilus asperatus (Bonsdorff, 1785) - árnyékkereső ormányos (DUDICH 1950, GozMány 1979, Podlussány 2007, 2009, 2010) 
Sciaphobus K. Daniel, 1904

Neosciaphobus Apfelbeck, 1922

Sciaphobus (Neosciaphobus) squalidus (Gyllenhal, 1834) - nagy szálkásbarkó (PoDlussánY 2014) - Other names: keleti gyümölcsfa-ormányos (GozmÁNY 1979); nagy csupaszbarkó (PoDLUSSÁNY 2007)

\section{Sciaphobus K. Daniel, 1904}

Sciaphobus (Sciaphobus) barbatulus (Germar, 1824) - smaragd-szálkásbarkó

Sciaphobus (Sciaphobus) caesius (Hampe, 1870) - fogas szálkásbarkó (Podlussány 2007, 2009)

Sciaphobus (Sciaphobus) scitulus (Germar, 1824) - füzöld szálkásbarkó (Podlussány 2007)

Sitonini Gistel, 1848

Charagmus Schönherr, 1826

Charagmus gressorius (Fabricius, 1792) (GYörgY \& PoDLussány 2005) pompás csipkézőbogár - Other names: nagy csillagfürt-csipkézőbogár (GoZMÁNY 1979)

Charagmus griseus (Fabricius, 1775) - szürke csipkézőbogár (JolSVAY et al. 1977, MER KL 2018, MERKL \& SzÉNÁsi 2018, MER KL et al. 2019) - Other names: csillagfürt-csipkézőbogár, szürke csipkézőbarkó (GozMÁNY 1979); szürke csipkézőbarkó (nagy csipkézőbogár, szürke kisbarkó) (SÁRINGER in JERMY \& BALÁZS 1990)

\section{Coelositona González, 1971}

Coelositona cambricus Stephens, 1831 - selymesszőrü csipkézőbogár - Other names: elegáns csipkézőbogár (PodlussánY 2007); kambriai aka (I. FRIVALDSZKY 1865)

Coelositona cinerascens Fåhraeus, 1840 - kerep-csipkézőbogár (SzÉNÁsI 2012) 


\section{Sitona Germar, 1817}

Sitona ambiguus Gyllenhal, 1834 - csíkos csipkézőbogár (PoDLussánY 2007)

Sitona callosus Gyllenhal, 1834 - szempillás csipkézőbogár (MERKL 2018, Merkl \& SZÉnÁsi 2018, Merkl et al.2019, Podlussány 2007, 2009, SzÉNÁsi 2012, SZÉNÁsi et al. 2016)

Sitona cylindricollis cylindricollis Fåhraeus, 1840 - hengerestorú csipkézőbogár (ENDRődi 1960, GoZMÁNY 1979, JOLSVAY et al. 1977, MER KL \& SZÉNÁSI 2018, MERKL et al. 2019, PodLussÁNy 2007, 2009, 2014, SÁRINGER in JERMY \& BALÁZs 1990, SZÉNÁSI 2012, SZÉNÁsi et al. 2016)

Sitona hispidulus (Fabricius, 1777) - szőrös csipkézőbogár (ENDRŐDI 1960, Gozmány 1979, Jolsvay et al. 1977, KaSZab 1969, Merkl \& SzÉnÁsi 2018, Podlussány 2007, 2009, 2014, SzÉnÁsi 2012, Tóth 1999) - Other names: mezei lucernabarkó Kadocsa 1929); szőrös kisbarkó (DUDICH 1950); mezei lucernabarkó, szőrös kisbarkó, szőrös csipkézőbarkó (GOZMÁNY 1979); szőrös csipkézőbarkó (BALÁs \& SÁRINGER 1984); szőrös csipkézőbarkó (mezei lucernabarkó, szőrös kisbarkó) (SÁRINGER in JERMY \& BALÁzs 1990)

Sitona humeralis Stephens, 1831 - lucerna-csipkézőbogár (ENDRöDI 1960, Gozmány 1979, Jolsvayet al. 1977, Kaszab 1969, Merkl 2018, Merkl \& SZÉnÁsi 2018, MERKL et al. 2019, Podlussány 2007, 2009, 2010, 2014, SÁRINGER in JERMY \& BALÁzs 1990, SZÉNÁSI 2012, SZÉNÁsI et al. 2016) Other names: lucerna-kisbarkó (DUDICH 1950); csipkézőbogár (UBRIZsy \& REICH ART 1958); lucerna-csipkéző, lucerna-kisbarkó (GozMÁ NY 1979); lucernacsipkézőbarkó (kis lucernacsipkéző) (SÁRINGER in JERMY \& BALÁZs 1990); lucerna-csipkézőbarkó (HAR MOs et al. 2002)

Sitona inops Schönherr, 1832 - laposszemü csipkézőbogár (MERKL \& SZÉNÁSI 2018, MER KL et al. 2019, PODLUSSÁNy 2007, 2009, SZÉNÁsi 2012)

Sitona languidus Gyllenhal, 1834 - koronafürt-csipkézőbogár (MER KL \& SZÉnÁsi 2018, MERKL et al. 2019, PodLussány 2007, 2009, 2014, SzÉNÁsi 2012, SZÉNÁsi et al. 2016)

Sitona lateralis Gyllenhal, 1834 (= ononidis Sharp, 1867) - iglicecsipkézőbogár (MERKL et al. 2019, Podlussány 2007, 2009, 2010, 2014, SZÉNÁsi 2012, SZÉNÁsI et al. 2016)

Sitona lineatus (Linnaeus, 1758) - sávos csipkézőbogár (ENDRőDI 1960, Gozmány 1979, Jolsvay et al. 1977, Kasza b 1969, Mer Kl \& Szénási 2018, MERKL et al. 2019, PodLussány 2007, 2009, 2010, 2014, SÁringer in JER MY \& BALÁzs 1990, SzÉNÁsi et al. 2016) - Other names: vonalas lucernabarkó (KAdocsa 1929); vonalas kisbarkó (Dudich 1950, PÉTERFI 1958); sávos kisbarkó, sávos kisbarkó, vonalas kisbarkó, vonalas csipkézőbarkó, vonalas lucernabarkó (GOZMÁNY 1979); lucernabarkó (Jolsvay et al. 1977); vonalas csipkézőbarkó (BALÁs \& SÁRINGER 1984); sávos csipkézőbarkó (vonalas csipkézőbarkó, vonalas lucernabarkó, vonalas kisbarkó) (SÁRINGER in JERMY \& BALÁzs 1990); sávos csipkézőbarkó (HARMOS et al. 2002, Tót H 1999) 
Sitona longulus Gyllenhal, 1834 - hosszú csipkézőbogár (ENDRŐDI 1960, Jolsvay et al. 1977, Merkl \& SzÉnÁsi 2018, Podlussány 2007, SÁringer in JERMY \& BALÁZs 1990, SZÉNÁSI 2012) - Other names: hosszú csipkézőbarkó (lucerna gyökérbarkó) (SÁRINGER in JERMY \& BALÁzs 1990); hosszú csipkézőbarkó (HAR Mos et al. 2002)

Sitona macularius macularius (Marsham, 1802) - borsó-csipkézőbogár (ENDRÖDi 1960, GoZMÁNY 1979, JolsvaY et al. 1977, KASZAB 1969, MER KL \& SZÉnÁsi 2018, MERKL et al. 2019, Podlussány 2007, 2009, 2010, 2014, SÁRINGER in JERMY \& BALÁzs 1990, SZÉNÁSI 2012, SZÉNÁSI $e t$ al. 2016, TóTH 1999) - Other names: szőrös lucernabarkó (KADOCSA 1929); sávosnyakú kisbarkó (DUDICH 1950, PÉTERFI 1958); csipkéző, sávosnyakú kisbarkó, sávosnyakú csipkézőbarkó szőrös lucernabarkó, sávosnyakú csipkézőbogár, borsócsipkéző, csipkéző bogár (GOZMÁNY 1979); sávosnyakú csipkézőbogár (PÉTERFI 1958); sávosnyakú csipkézőbarkó (BALÁs \& SÁRINGER 1984); borsó-csipkézőbarkó (szőrös lucernabarkó, sávosnyakú kisbarkó, sávosnyakú csipkézőbogár) (SÁRINGER in JERMY \& BALÁZS 1990)

Sitona obsoletus obsoletus (Gmelin, 1790) (= flavescens Marsham, 1802; = lepidus Gyllenhal, 1834) - sárga csipkézőbogár (ENDRőDI 1960, GOZMÁNY 1979, Jolsvay et al. 1977, Podlussány 2007, 2009, 2010) - Other names: sárga csipkézőbarkó (sárga csipkézőbogár, sárgás kisbarkó) (SÁRINGER in JERMY \& BALÁZs 1990)

Sitona puncticollis Stephens, 1831 - nagy csipkézőbogár (ENDRődi 1960, Gozmány 1979, Jolsvay et al. 1977, Kaszab 1969, Merkl 2018, Merkl \& SZÉnÁsi 2018, MER Kl \& Vig 2009, MER KL et al. 2019, PodLuSSÁny 2007, 2009, 2014, SÁRINGER in JERMY \& BALÁZs 1990, SZÉNÁSI 2012, SZÉNÁsI et al. 2016) Other names: nagy lucerna-csipkéző (GOZMÁNY 1979); nagy csipkézőbarkó (ragyásnyakú lucernabarkó) (SÁRINGER in JERMY \& BALÁzS 1990)

Sitona striatellus Gyllenhal, 1834 (= tibialis Herbst, 1795) - bükkönycsipkézőbogár (ENDRődi 1960, GozmánY 1979, Jolsvay et al. 1977, KASZAB 1969, Merkl \& SzÉnÁsi 2018, MERkl et al. 2019, Podlussány 2007, 2009, SÁRINGER in JERMY \& BALÁzS 1990, SZÉNÁsi 2012, SzÉNÁsI et al. 2016); seprőzanót-kisbarkó (DUDICH 1950, GOZMÁNY 1979); bükköny-csipkézőbarkó (seprőzanót kisbarkó) (SÁRINGER in JERMY \& BALÁzs 1990)

Sitona sulcifrons argutulus Gyllenhal, 1834 - szegélyes csipkézőbogár (ENDRődi 1960, Gozmány 1979, Jolsvay et al. 1977, KASZA B 1969, MER KL \& Vig 2009, Merkl et al. 2019, Podlussány 2007, 2009, 2010, 2014, SÁringer in JERMY \& BALÁzs 1990, SZÉNÁSI 2012, SZÉNÁsi et al. 2016) - Other names: szegélyes kisbarkó (DUDICH 1950); szegélyes csipkéző, szegélyes kisbarkó, sávos csipkézőbogár (GOZMÁNY 1979); szegélyes csipkézőbarkó (szegélyes kisbarkó) (SÁRINGER in JERMY \& BALÁZs 1990)

Sitona suturalis Stephens, 1831 - fémes csipkézőbogár - Other names: színes csipkézőbogár (MER KL \& SZÉnÁsi 2018, MER KL et al.2019, PODLUSSÁNy 2007, 
2009, 2010, 2014, SZÉNÁsi 2012); bab csipkézőbarkó (Harmos et al. 2002, SÁRINGER in JERMY \& BALÁzS 1990)

Sitona waterhousei waterhousei Walton, 1846 - dülledtszemü csipkézőbogár (Merkl 2018, Merkl \& Szénási 2018, Podlussány 2007, 2009, 2014) Other names: Waterhouse-csipkézőbogár (Podlussány 2010, SzÉNÁsi 2012, SZÉNÁsi et al. 2016)

Tanymecini Lacordaire, 1863

Chlorophanus C. R. Sahlberg, 1823

Chlorophanus excisus (Fabricius, 1801) - kerektorú zöldormányos (MERKL \& SZÉnÁsi 2018, Podlussány 2007) - Other names: kiívelt zöldény (I. FRIVALDSZKY 1865); csalárd zöldény (I. FRIVALDSZKY 1865)

Chlorophanus flavescens (Fabricius, 1787) (= graminicola Schönherr, 1832) nagy zöldormányos (DUDICH 1950)

Chlorophanus pollinosus (Fabricius, 1792) (= gibbosus Paykull, 1792) - kis zöldormányos (DUDICH 1950)

Chlorophanusviridisbalcanicus Behne, 1989 (DieckM ANN \& BeHNe 1994)balkáni zöldormányos

Chlorophanusviridisviridis(Linnaeus, 1758) - sárgaszegélyeszöldormányosOther names: közönséges zöldormányos (GozmánY 1979)

Cycloderes Sahlberg, 1823

Cycloderes Sahlberg, 1823

Cycloderes (Cycloderes) pilosulus (Herbst, 1795) (= pilosus Fabricius, 1794) pikkelyeslábú ormányos (DUdich 1950, DUdich \& LoKsA 1969, GozmánY 1979, Mer Kl 2018, Merkl \& SzénÁsi 2018, Merkl et al. 2019, Podlussány 2007, 2014, SZÉNÁsI 2012, SZÉNÁsI et al. 2016)

Tanymecus Germar, 1817

Episomecus Reitter, 1903

Tanymecus (Episomecus) dilaticollis Gyllenhal, 1834 - kukoricahegyesfarúbarkó (MERKL et al. 2019) - Other names: kukoricabarkó (ENDRŐDI 1960, Harmos etal. 2002, Kasza B 1969, MANNINGER 1960, PodLussánY 2007, 2014, UBRIZSY \& REICHA RT 1958); kisbarkó (MANNINGER 1960); kukoricabarkó (kisbarkó, déli szürkebarkó, szürke kukoricabarkó, kis hegyesfarú-barkó) (SÁRINGER in JERMY \& BALÁZS 1990) 


\section{Tanymecus Germar, 1817}

Tanymecus (Tanymecus) palliatus (Fabricius, 1787) - közönséges hegyesfarúbarkó (MERKL et al. 2019) - Other names: hegyesfarkú répabarkó (DUDICH 1950); hegyesfarú répabarkó (GOZMÁNY 1979, JABLONOWSKI 1906, Kaszab 1969, Podlussány 2007, 2009, Ubrizsy \& Reichart 1958); hegyesfarú barkó (BALÁs 1963, BALÁs \& SÁRINGER 1984, ENDRŐDI 1960, GoZmány 1979, Harmos et al. 2002, Jolsvay et al. 1977, MANNINGER 1960, SZÉNÁsi 2012, SZÉNÁsI et al. 2016, PODLUSSÁNY 2010, 2014); hegyesfarú barkó (MERKL \& SZÉNÁsI 2018, SÁRINGER in JERMY \& BALÁzS 1990)

Trachyphloeini Gistel, 1848

Cathormiocerus Schönherr, 1842

Cathormiocerus aristatus (Gyllenhal, 1827) - szálkás éjiormányos (DUDICH 1950, Merkl \& SzÉnÁsi 2018, Merkl et al. 2019, Podlussány 2007, 2009, 2010, 2014, SZÉNÁSI 2012, SZÉNÁsI et al. 2016)

Cathormiocerus spinosus (Goeze, 1777) (= asperatus Boheman, 1842; = olivieri Bedel, 1883) - apró éjiormányos

Pelletierellus Borovec, 2009

Pelletierellus ventricosus (Germar, 1824) - tarka éjiormányos (KASZA в 1969, PODLUSSÁNY 2007, 2014) - Other names: pohos csülköny (I. FrIVALDSZKY 1865)

\section{Romualdius Borovec, 2009}

Romualdius scaber (Linnaeus, 1758) (= bifoveolatus Beck, 1817; = angustisetulus V. Hansen, 1915) - laposszemü éjiormányos (MERKL et al. 2019, PODLUSSÁNY 2007)

Stuebenius Borovec, 2009

Stuebenius frivaldszkyi (Kuthy, 1887) - Frivaldszky-éjiormányos (MERKL \& SZÉNÁsi 2018, MER KL \& VIG 2009, MER Kl et al. 2019, SZÉNÁsi 2012, SZÉNÁSI et al. 2016) 
Trachyphloeus Germar, 1817

Trachyphloeus Germar, 1817

Trachyphloeus (Trachyphloeus) alternans Gyllenhal, 1834 - bordás éjiormányos (Dudich 1950, Harmos et al. 2002, MERKL \& SzÉnÁsi 2018, Podlussány 2007, 2014, SZÉNÁsi 2012, SZÉNÁsi et al. 2016)

Trachyphloeus (Trachyphloeus) parallelus Seidlitz, 1868 - pompás éjiormányos (PodLUSSÁNy 2007)

Trachyphloeus (Trachyphloeus) scabriculus (Linnaeus, 1771) - bunkósszőrü éjiormányos (DUdich 1950, GoZMÁNY 1979, PODLUSSÁNy 2007)

Trachyphloeus (Trachyphloeus) spinimanus Germar, 1824 - töviseslábú éjiormányos (MER KL 2018, MER KL \& SZÉnÁsi 2018, PodlusSÁnY 2007, 2014, SZÉNÁSI 2012)

\title{
Hyperinae Marseul, 1863 \\ Hyperini Marseul, 1863 \\ Brachypera Capiomont, 1868 \\ Antidonus Bedel, 1886
}

Brachypera (Antidonus) dauci (Olivier, 1807) (= fasciculatus Herbst, 1795) gólyaorr-gubósormányos (MER KL 2018, MER KL \& SZÉNÁsi 2018, PodLUSSÁNY 2007, 2014, SZÉNÁsI 2012, SzÉNÁsI et al. 2016)

Brachypera (Antidonus) zoilus (Scopoli, 1763) - lucerna-gubósormányos (Dudich 1950, Dudich \& LOKSA 1969, GoZM ÁNY 1979, MER KL \&SZÉNÁsi2018, Merkl \& Vig 2009, Podlussány 2007, 2009, SÁringer in Jermy \& BALÁzs 1990, SZÉNÁsi 2012, SZÉNÁsi et al. 2016) - Other names: vöröshere-ormányos (SÁRINGER in JERMY \& BALÁzS 1990)

Coniatus Germar, 1817

Bagoides Capiomont, 1868

Coniatus(Bagoides)splendidulus(Fabricius, 1781)(PodLussány etal.2016)fémeszöld tamariskaormányos

\author{
Donus Jekel, 1865 \\ (= Neoglanis Alonso-Zarazaga et Lyal, 1999)
}

Donus comatus (Boheman, 1842) - szálkás gubósormányos

Donus elegans (Boheman, 1842) - pettyeshátú gubósormányos - Other names: nyalka növenyész (I. FRIVA LDSZKY 1865) 
Donus intermedius intermedius (Boheman, 1842) - menta-gubósormányos

Donus ovalis (Boheman, 1842) - tojásdad gubósormányos

Donusoxalis(Herbst, 1795) (=oxalidisHerbst, 1795) -sóska-gubósormányosOther names: sósdi növenyész (I. FRIVALDSZKY 1865); sóska-szálkásormányos (Podlussány 2009, Merkl \& Vig 2009)

Donus palumbarius (Germar, 1821) - martilapu-gubósormányos

Donus tessellatus (Boheman, 1834) (= maculatus W. Redtenbacher, 1842) ökörfarkkóró-gubósormányos - Other names: koczkás növenyész (I. FRIVALDSZKY 1865); nagy ökörfarkkóróormányos (DUDICH 1950, GozMÁNY 1979); cickafark-szálkásormányos (MER KL et al. 2019, SZÉNÁsI 2012, SzÉNÁsI et al. 2016, PoDlussáNy 2014); ökörfarkkóró-szálkásormányos (MERKL 2018, MERKL \& SZÉNÁSI 2018)

Donus velutinus (Boheman, 1842) - molyhos gubósormányos (I. FRIVALDSZKY 1865) - Other names: molyhos növenyész (I. FRIVALDSZKY 1865)

Donus viennensis (Herbst, 1795) - bécsi gubósormányos (I. FRIVALDSZKY 1865) - Other names: bécsi növenyész (I. FRIVALDSZKY 1865)

\section{Herpes Bedel, 1874}

Herpes porcellus (Lacordaire, 1863) - bütyköshátú ormányos (MERKL \& SZÉNÁsi 2018, MERKL \& VIG 2009)

\section{Hypera Germar, 1817 \\ Boreohypera Korotyaev, 1999}

Hypera (Boreohypera) diversipunctata (Schrank, 1798) (= elongatus Paykull, 1792) - nyúlánk pikkelyesormámyos - Other names: hosszú pikkelyesormányos (PodlussánY 2007); különleges pikkelyesormányos (SZÉNÁsı et al. 2016)

\section{Dapalinus Capiomont, 1868}

Hypera (Dapalinus) contaminata (Herbst, 1795) - homályos pikkelyesormányos (Podlussány 2009, SZÉNÁsi et al. 2016) - Other names: piszkos pikkelyesormányos (PodLussánY 2007, 2014)

Hypera (Dapalinus) fornicata (Penecke, 1928) - domború pikkelyesormányos (Podlussány 2007)

Hypera (Dapalinus) meles (Fabricius, 1792) - lóhere-pikkelyesormányos (MER KL 2018, MERKL \& SZÉNÁsi 2018, SZÉNÁsi 2012, SZÉNÁsi et al. 2016) Other names: herevirág-gubósormányos (GOZMÁNY 1979); lóhere-gubósormányos (PodLussány 2007, 2010, 2014, SÁRINGER in JERMy \& BALÁzs 1990) 
Hypera (Dapalinus) striata (Boheman, 1834) - sávos pikkelyesormányos (PodLUSSÁNY 2007, 2010, 2014, SZÉNÁsi 2012, SZÉNÁsi et al. 2016)

\section{Eririnomorphus Capiomont, 1868}

Hypera (Eririnomorphus) arundinis (Paykull, 1792) - békakorsópikkelyesormányos - Other names: békakorsó-gubósormányos (PoDLUSSÁNY 2007)

Hypera (Eririnomorphus) conmaculata (Herbst, 1795) (= adspersa Fabricius, 1792; = pollux Fabricius, 1801) - mocsári pikkelyesormányos (PoDlussánY 2009) - Other names: mocsári gubósoemányos (SzÉNÁsI et al. 2016)

Hypera(Eririnomorphus) libanotidis(Reitter,1896)(PodLussá NY etal.2017)tömjénillat-pikkelyesormányos

Hypera (Eririnomorphus) rumicis (Linnaeus, 1758) - sóskapikkelyesormányos (MERKL \& SzÉNÁsi 2018, PoDlusSÁnY 2009, SZÉnÁsi 2012) - Other names: sóska-gubósormányos (Dudich 1950, GozmánY 1979, PODLUSSÁNY 2007); sóskaormányos (sóskagubósormányos) (SÁRINGER in JERMY \& BALÁzs 1990); sóskaormányos (HARMOs et al. 2002)

\section{Hypera Germar, 1817 \\ (= Phytonomus Schönherr, 1823)}

Hypera (Hypera) cumana (Petri, 1901) - kun pikkelyesormányos

Hypera (Hypera) denominanda (Capiomont, 1868) - zömök pikkelyesormányos - Other names: neves pikkelyesormányos (PoDLUSSÁNY 2007)

Hypera (Hypera) melancholica (Fabricius, 1792) (= fuscocinerea Marsham, 1802; = murina Fabricius, 1792) - barnafoltos pikkelyesormányos - Other names: szürke gubósormányos (GozMÁNY 1979)

Hypera (Hypera) miles (Paykull, 1792) (= pedestris Paykull, 1792; = suspiciosa Herbst, 1795) - lednek-pikkelyesormányos (MER KL \& SzÉNÁsı 2018, Podlussány 2009) - Other names: lednek-gubósormányos (DUdich 1950, Podlussány 2007, SzÉNÁsi et al. 2016)

Hypera (Hypera) nigrirostris (Fabricius, 1775) - pompás pikkelyesormányos (MERKL \& SZÉNÁsi 2018, MERKL et al. 2019) - Other names: lóheregubósormányos (Dudich 1950, Dudich \& LoKsa 1969, Gozmány 1979, PoDlussáNY 2007) - Other names: iglice-pikkelyesormányos (SzÉNÁsI 2012); lóhere-ormányos (feketefejű lucernalevélrágó, feketeormányú lóheregubósormányos) (SÁRINGER in JERMY \& BALÁZS 1990); pompás gubósormányos (Podlussány 2010) 
Hypera (Hypera) plantaginis (DeGeer, 1775) - útifü-pikkelyesormányos (Merkl \& Szénási 2018, Podlussány 2009, SzÉnási 2012) - Other names: útifügubósormányos (DUDICH 1950, PoDLUSSÁNy 2007)

Hypera (Hypera)postica (Gyllenhal, 1813) (=variabilis Herbst, 1795)-lucernapikkelyesormányos (MER KL 2018, MER KL et al. 2019, PodLUSSÁNy 2009, 2014, SZÉNÁsi 2012, SZÉNÁsI et al. 2016) - Other names: változékony gubósormányos (DUdich 1950, GozMÁNY 1979); lucernaormányos (ENDRöDi 1963, GozMÁNY 1979, Harmos et al. 2002, MaNNINGer 1960, Ubrizsy \& ReICHART 1958); lucernaormányos (lucerna levélrágó, változékony gubósormányos) (SÁRINGER in JERMY \& BALÁzs 1990); közönséges lucernaormányos (PODLUSSÁNY 2007)

Hypera (Hypera) transsilvanica (Petri, 1901) - erdélyi pikkelyesormányos

Hypera (Hypera) venusta (Fabricius, 1781) (= trilineata Marsham, 1802) bíborhere-pikkelyesormányos (SzÉNÁsI 2012) - Other names: bíborhereormányos (ENDRöDi 1963, Jolsvay etal. 1977, KASZA B 1969, MANNINGER 1960, SÁRINGER in JERMY \& BALÁzs 1990, PODLUSSÁNY 2007)

Hypera (Hypera) viciae (Gyllenhal, 1813) - bükköny-pikkelyesormányos (MER KL etal.2019, SzÉNÁsi 2012, MER KL \& SZÉNÁsi 2018, PodLuSSÁNy 2014) Other names: bükkönygubósormányos (DUDICH 1950, Harmos et al. 2002, PODLUSSÁNy 2007, SÁRINGER in JERMY \& BALÁzs 1990)

Kippenbergia Alonso-Zarazaga, 2005

Hypera (Kippenbergia) arator (Linnaeus, 1758) - szegfü-pikkelyesormányos (MERKL 2018, MerKL \& SZÉNÁsi 2018, MerKL et al. 2019) - Other names: közönséges gubósormányos (BALÁs \& SÁRINGER 1984, DUDICH 1950, DUDICH \& LOKSA 1969, Gozmány 1979, HARmos et al. 2002, SÁrINGER in JERMy \& BALÁzs 1990, Podlussány 2007); közönséges pikkelyesormányos (MERKL \& Vig 2009, PodLUSSÁNY 2009, 2014, SZÉNÁSI 2012, SZÉNÁsi et al. 2016)

\section{Limobius Schönherr, 1843}

Limobius borealis borealis (Paykull, 1792) - szörös gólyaorrormányos (Merkl et al. 2019, Podlussány 2007, 2014, SzÉnÁsi 2012) - Other names: gólyaorrormányos (DUDICH 1950, HARmos et al. 2002)

\section{Metadonus Capiomont, 1868}

Metadonus distinguendus (Boheman, 1842) - keleti pikkelyesormányos (MERKL \& SZÉNÁSI 2018) 
Pachypera Capiomont, 1868

Pachypera kraatzi (Capiomont, 1868) - lapostorú pikkelyesormányos

Lixinae Schönherr, 1823

Cleonini Schönherr, 1826

Adosomus Faust, 1904

Adosomus Faust, 1904

Adosomus (Adosomus) roridus (Pallas, 1781) - fehérfoltos ürömbarkó Other names: deres dicser (I. FRIVALDSZKY 1865); ürömbarkó (PoDLUSSÁNY 2007)

\section{Aspropartbenis Gozis, 1886}

Asproparthenis punctiventris (Germar, 1824) - lisztes répabarkó (DUDICH 1950, DU DICH \& LOKSA 1969, EMICH 1899, ENDRőDI 1960, MANNINGER 1960, MANNINGER in JERMY \& BALÁzs 1990, MERKL \&VIG 2009, HARMOSetal.2002, SZÉNÁSi 2012, UbRIZSY \& REICHART 1958) - Other names: hosszúfejü bogár, nagy barkó, ormány répabarkó, répabarkó, répaormányos, répabogár (MANNINGER in JERMY \& BALÁZS 1990)

\section{Bothynoderes Schönherr, 1823}

Bothynoderes affinis (Schrank, 1781) (= fasciatus O. F. Müller, 1776) - sávos barkó (ENDRŐdi 1960, MER KL 2018, MER KL \& SZÉNÁsi 2018, SZÉNÁsi 2012) Other names: sávos répabarkó (ENDRŐDI 1960, KASZAB 1969, MANNINGER 1960, MANNINGer in Jermy \& BALÁzs 1990, Mer Kl \& Vig 2009, PodlussánY 2007); cickafarkbarkó, sávos répabarkó, sávoshátú répabarkó (GoZMÁNY 1979)

Bothynoderes declivis (Olivier, 1807) - libatopbarkó (SzÉNÁsI 2012, MERKL 2018, MERKL \& SZÉNÁsi 2018) - Other names: kajlajegyü dicser (I. FRIVALDSZKY 1865); ázsiai répabarkó (GoZMÁNY 1979)

\section{Cleonis Dejean, 1821}

Cleonispigra (Scopoli, 1763) - közönséges bogáncsbarkó (MER KL \& SzÉNÁsI 2018, MER KL et al.2019) - Other names: bogáncsbarkó (DUDICH 1950, DUDICH \& LoKsa 1969, Gozmány 1979, Jolsvay et al. 1977, PodLUSSÁnY 2007, 2009, 2014, 
SZÉNÁsi 2012, SZÉNÁsi et al. 2016); bogáncsbarkó (barázdásfejü répabarkó) (MANNINGER in JeRMY \& BALÁZs 1990)

Coniocleonus Motschulsky, 1860

Augustecleonus Arzanov, 2006

Coniocleonus (Augustecleonus) turbatus (Fåhraeus, 1842) (= glaucus Fabricius, 1787) - homoki barkó (MERKL 2018) - Other names: nagy szürke ormányosbogár (GoZMÁNY 1979)

Plagiographus Chevrolat, 1869

Coniocleonus (Plagiographus) cicatricosus (Hoppe, 1795) - ripacsos barkó

Coniocleonus (Plagiographus) excoriatus (Gyllenhal, 1834) - horzsolt barkó

Coniocleonus (Plagiographus) nigrosuturatus (Goeze, 1777) - nyíljegyes barkó (DUDICH 1950, ENDRŐdi 1960, GoZMÁNY 1979, MER KL \& SZÉNÁSI 2018, MERKL \& VIg 2009, SZÉNÁsI 2012, SzÉNÁsI et al.2016) - Other names: karcsú répabarkó (ENDRŐDI 1960, GoZMÁNY 1979)

\section{Cyphocleonus Motschulsky, 1860}

Cyphocleonus achates (Fåhraeus, 1842) - ékes imolabarkó - Other names: ágát dicser (I. FRIVALDSZ KY 1865); imolabarkó (PODLUSSÁNY 2007)

Cyphocleonus dealbatus (Gmelin, 1790) (= tigrinus Panzer, 1781) - márványos barkó (Dudich 1950, GozmÁNy 1979, MER KL 2018, MER KL \& SZÉNÁsi 2018, MERKL \& Vig 2009, Podlussány 2007, 2009, 2014, SzÉNÁsi 2012, SzÉNÁsi et al. 2016)

Cyphocleonus trisulcatus (Herbst, 1795) - kockás margarétabarkó - Other names: hegyi répabarkó (GoZMÁNY 1979); margarétabarkó (PoDLUSSÁN Y 2007)

Leucophyes Marshall, 1946

(= Leucosomus Motschulsky, 1860)

Leucophyes pedestris (Poda, 1761) - négypettyes barkó (DUDICH 1950, MERKL \& SZÉNÁSI 2018) 


\section{Mecaspis Schönherr, 1823}

Mecaspis alternans (Herbst, 1795) - keserügyökér-barkó (DUDICH 1950, Dudich \& LoKsa 1969, Podlussány 2007, 2009, 2014)

Mecaspis striatella (Fabricius, 1792) - barázdás barkó - Other names: rejtődző dicser (I. FRIVALDSZKY 1865)

\section{Pachycerus Schönherr, 1823}

Pachycerus madidus (Olivier, 1807) - tarka barkó (ENDRÖDI 1960) - Other names: tarka répabarkó (ENDRŐDI 1960, PODLUSSÁNY 2007)

Pachycerus segnis (Germar, 1824) (= cordiger Germar, 1818) - hamuszínü barkó

Pseudocleonus Chevrolat, 1872

Neopseudocleonus Arzanov, 2005

Pseudocleonus(Neopseudocleonus) grammicus(Panzer, 1789)-bábakalácsbarkó

Pseudocleonus Chevrolat, 1872

Pseudocleonus (Pseudocleonus) cinereus (Schrank, 1781) - hamvas barkó (ENDRŐDI 1960,MER KL \&SZÉNÁSI2018,PODLUSSÁNY2009,2014,SZÉNÁSI2012, SZÉNÁsi et al. 2016) - Other names: hamvas répabarkó (ENDRődi 1960, Gozmány 1979, Harmosetal. 2002, PODLUSSÁNy 2007, SÁR INGER in JER MY \& BALÁZS 1990)

Rhabdorrbynchus Motschulsky, 1860 (= Pachycerus Schönherr, 1826)

Rhabdorrhynchusechii Brahm, 1790 (=varius Herbst, 1795) - kígyósziszbarkó (MERKL \& SZÉNÁsi 2018, Podlussány 2007)

Stephanocleonus Motschulsky, 1860

Sanzia Alonso-Zarazaga et Lyal, 1999

Stephanocleonus (Sanzia) microgrammus (Gyllenhal, 1834) (PodLussánY 2001b) - jövevény barkó 
Lixini Schönherr, 1823

Lachnaeus Schönherr, 1826

Lachnaeus crinitus Schönherr, 1826 - réti sünbarkó (MER KL 2018, MER KL \& SZÉNÁsi 2018) - Other names: sünbarkó(MER KL \& Vig 2009, PODLUSSÁNY 2007, SZÉNÁsi 2012, SZÉNÁsi et al. 2016)

Larinus Dejean, 1821

Larinomesius Reitter, 1924

Larinus (Larinomesius) canescens Gyllenhal, 1835 - déli púderbarkó (MERKL \& VIG 2009)

Larinus(Larinomesius) minutus Gyllenhal, 1835-aprópúderbarkó(MER KL \& SzÉnÁsi 2018, MERKL et al. 2019, Podlussány 2014)

Larinus(Larinomesius) nubeculosus Gyllenhal,1835(=rugulosusPetri, 1907)tömzsi púderbarkó

Larinus (Larinomesius) obtusus Gyllenhal, 1835 - imola-púderbarkó (MER KL \& SZÉnÁsi 2018, MER KL et al.2019, PodlusSÁNY 2014) - Other names: tompa vastang (I. FRIVALDSZKY 1865); imola-púderbogár (PoDLUSSÁNY 2007, 2009, 2010, SZÉNÁsI 2012, SZÉNÁsI et al. 2016)

Larinus (Larinomesius) syriacus Gyllenhal, 1835 (KRÁt KÝ \& PodLUssánY 2008) - szír púderbarkó (MERKL \& SZÉNÁsi 2018)

\section{Larinus Dejean, 1821}

Larinus (Larinus) latus (Herbst, 1783) - óriás-púderbarkó - Other names: bogácsi vastang (I. FRIVALDSZKY 1865)

Larinus (Larinus) pollinis (Laicharting, 1781) (= brevis Herbst, 1795) bábakalács-púderbarkó (MER KL \& SZÉNÁsi 2018, PoDLUSSÁNY 2007) - Other names: agastyán vastang (I. FRIVALDSZKY 1865) - Other names: púderbogár (GOZMÁNY 1979)

\section{Phyllonomeus Gistel, 1856}

Larinus (Phyllonomeus) carlinae (Olivier, 1807) (Alonso-ZarazAGA et al. 2017) - foltos púderbarkó (PodLussány 2009, MER KL \& SzÉNÁsi 2018) Other names: foltos púderbogár (HARmos et al. 2002, Podlussány 2007, SZÉNÁSI 2012, SZÉNÁsI et al. 2016)

Larinus (Phyllonomeus) centaurii (Olivier, 1807) (= beckeri Petri, 1907) szürke púderbarkó 
Larinus (Phyllonomeus) iaceae (Fabricius, 1775)(= jaceae Fabricius, 1792) sávosnyakú púderbarkó (DUDICH 1950, DUDICH \& LoKsA 1969, MER KL 2018, MER KL \& SzÉNÁsi 2018, PodlussánY 2009, 2014) - Other names: sávosnyakú púderbogár (Gozmány 1979, Harmos et al. 2002, PodLUSSÁNy 2007, SZÉNÁsI 2012, SZÉNÁsI et al. 2016)

Larinus (Phyllonomeus) sturnus (Schaller, 1783) - aszat-púderbarkó (Dudich 1950, MERKL 2018, Merkl \& SzénÁsi 2018) - Other names: aszatpúderbogár (DUdich 1950, Dudich \& LoKsa 1969, GozmánY 1979, Harmos et al. 2002, Jolsvay et al. 1977, Podlussán y 2007, SZÉnÁsi 2012)

Larinus (Phyllonomeus) turbinatus Gyllenhal, 1835 - kúposorrú púderbarkó (MER KL \& SzÉnÁsi 2018, MER KL \& Vig 2009, MER Kl et al. 2019, Podlussány 2007, 2009, 2014) - Other names: egyenesorrú puderbogár (DUDICH 1950, DUDICH \& LOKSA 1969, HARMOs et al. 2002); kúposorrú púderbogár (Podlussány 2007, 2010, SZÉNÁsi 2012, SZÉNÁsi et al. 2016)

Lixus Fabricius, 1801

Broconius Desbrochers des Loges, 1904

Lixus (Broconius) rubicundus Zoubkoff, 1833 (=flavescens Boheman, 1835) laboda-dudvabarkó (PoDlussáNY 2007) - Other names: sárgás hosszancs (I. FRIVALDSZKY 1865)

Callistolixus Reitter, 1916

Lixus (Callistolixus) cylindrus (Fabricius, 1781) - cifra dudvabarkó - Other names: hengerded hosszancs (I. FRIVALDSZKY 1865)

Compsolixus Reitter, 1916

Lixus (Compsolixus) albomarginatus Boheman, 1842 (= apfelbecki Petri, 1904) - fehérszegélyes dudvabarkó (MERKL 2018, MERKL \& SzÉNÁsi 2018, Podlussány 2007, 2014) - Other names: fehérszegélyü hosszancs (I. FRIVALDSZKY 1865)

Lixus (Compsolixus) ochraceusBoheman, 1842-retek-dudvabarkó(MER KL \& SZÉNÁSI 2018, PODLUSSÁNY 2007, 2014, SZÉNÁSI 2012) 


\section{Dilixellus Reitter, 1916}

Lixus (Dilixellus) bardanae (Fabricius, 1787) (PodLussány 2001b) - lóromdudvabarkó (Merkl \& SzÉNÁsi 2018, Podlussány 2007, SZÉnÁsi 2012) Other names: sóskafúró ormányos (GozMÁNY 1979)

Lixus (Dilixellus) euphorbiae Capiomont, 1875 - kutyatej-dudvabarkó (MERKL et al. 2019, PodLussánY 2007)

Lixus (Dilixellus) fasciculatus Boheman, 1835 - nyurgalábú dudvabarkó (MERKL \& SZÉNÁsi 2018, PODLUSSÁNY 2007)

Lixus (Dilixellus) linearis Olivier, 1807 (= cribricollis Boheman, 1835) sóska-dudvabarkó (PoDlussánY 2007)

Lixus (Dilixellus) pulverulentus (Scopoli, 1763) (= algirus Fabricius, 1801; = angustatus Fabricius, 1775)-mályvafúró dudvabarkó (MER KL \& SzÉNÁsı 2018, PodLussánY 2007, SZÉNÁsi 2012) - Other names: mályvafúró (DUDICH 1950, GoZMÁNY 1979)

Lixus (Dilixellus) punctiventris Boheman, 1835 - aggófü-dudvabarkó (Merkl \& SzÉnÁsi 2018, Podlussány 2007)

\section{Epimeces Billberg, 1820}

Lixus (Epimeces) cardui Olivier, 1807 - bogáncsfúró dudvabarkó (MER KL \& Vig 2009, PoDLusSÁN Y 2007, SZÉNÁsi 2012) - Other names: hímporos hosszancs (I. FRIVALDSZKY 1865); zömök bógáncsfúró (Dudich 1950, Dudich \& LoKsa 1969, Jolsvay et al. 1977, Ha RMos et al. 2002)

Lixus (Epimeces) filiformis (Fabricius, 1781) (= elongatus Goeze, 1777) karcsú dudvabarkó (MER KL 2018, MER KL \& SZÉnÁsi 2018, PODLUSSÁNY 2007, 2009, 2014, SZÉNÁsi 2012) - Other names: nyúlánk hosszancs (I. FRIVALDSZKY 1865); karcsú bógáncsfúró (Dudich 1950, Dudich \& LoKSA 1969, HaRmos et al. 2002, JolsvaY et al. 1977)

\section{Eulixus Reitter, 1916}

Lixus (Eulixus) iridis Olivier, 1807 - bürökfúró dudvabarkó (MER KL \& VIG 2009, Podlussány 2007, 2009, 2014) - Other names: bürökfúró (DUDICH 1950, Dudich \& Loksa 1969, Gozmány 1979, Jolsvay et al. 1977)

Lixus (Eulixus) lateralis (Panzer, 1789) - ternye-dudvabarkó - Other names: ternyebarkó (BALÁs \& SÁRINGER 1984, SÁRINGER in JER MY \& BALÁzs 1990)

Lixus (Eulixus) myagri Olivier, 1807 - pöttyöshasú dudvabarkó (SzÉNÁsI et al. 2016) 
Lixus (Eulixus) punctirostris Boheman, 1842 - pontozottorrú dudvabarkó (Podlussány 2007, 2009, SzÉnÁsi 2012) - Other names: pontozottcsőrü hosszancs (I. FRIVALDSZKY 1865)

\section{Lixus Fabricius, 1801}

Lixus (Lixus) paraplecticus (Linnaeus, 1758) - fecskefarkú dudvabarkó (Dudich 1950, Dudich \& Loksa 1969, Jolsvay et al. 1977, Mer KL \& SzÉNÁsi 2018, Merkl \& Vig 2009, Podlussány 2007, SzÉNÁsi 2012) - Other names: villáshegyủ dudvafúró, fecskefarkú dudvafúró (GozmÁNY 1979)

\section{Ortholixus Reitter, 1916}

Lixus (Ortholixus) angustus (Herbst, 1795) - rövid dudvabarkó (Podlussány 2007, SZÉnÁsi 2012) - Other names: szegfübarkó (GoZmánY 1979, KASZAB 1969, MANNINGER in JeRMY \& BALÁzS 1990)

Lixus (Ortholixus) bituberculatus Smreczyński, 1968 - kétpúpú dudvabarkó (Podlussány 2014)

Lixus (Ortholixus) cinerascens Schönherr, 1832 (= elegantulus Boheman, 1842) - hamvas dudvabarkó (MERKL \& SZÉNÁsi 2018) - Other names: csínos hosszancs (I. FRIVALDSZKY 1865)

Lixus (Ortholixus) tibialis Boheman, 1842 - hölgymál-dudvabarkó

Lixus (Ortholixus) vilis (Rossi, 1790) - gémorr-dudvabarkó (MERKL 2018, MERKL \& SZÉNÁSI 2018)

\section{Phillixus Petri, 1904}

Lixus (Phillixus) brevipes C. N. F. Brisout de Barneville, 1866 - vaskosorrú dudvabarkó(MER KL\&SZÉNÁsi2018, MERKLetal.2019,PODLUSSÁNY2007,2014, SZÉNÁsi 2012, SZÉNÁsI et al. 2016)

Lixus (Phillixus) scabricollis Boheman, 1842 - répalevél-dudvabarkó - Other names: répa-levélbarkó (répalevélfúró, répa-levélormányos, réti-dudvafúró) (MANNINGER in JERMY \& BALÁzS 1990)

Lixus (Phillixus) subtilis Boheman, 1835 - disznóparéj-dudvabarkó (Podlussány 2007, SzÉNÁsi 2012, SzÉnÁsi et al. 2016) - Other names: vézna hosszancs (I. FRIVALDSZKY 1865) 


\title{
Rhinocyllus Germar, 1817
}

Rhinocyllus conicus (Froelich, 1792) - tömpeorrú barkó (MER KL \& SzÉNÁsI 2018, PodLUSSÁNy 2007, 2009, SZÉnÁsi 2012) - Other names: fogfájásmulasztó ormányos (DUDICH 1950, HARMos et al. 2002)

\author{
Mesoptiliinae Lacordaire, 1863 \\ Magdalidini Pascoe, 1870 \\ Magdalis Germar, 1817 \\ Edo Germar, 1819
}

Magdalis(Edo) nitidipennis Boheman, 1843 - tömpeorrú magdolnaormányos (MERKL et al. 2019) - Other names: tömpeorrú nyárormányos (PODLUSSÁNY 2007)

Magdalis (Edo) ruficornis (Linnaeus, 1758) - sárgacsápú magdolnaormányos (MERKL \& SzÉNÁSI 2018, MERKL et al. 2019) - Other names: sárgacsápú gyümölcsfaormányos(DUdich 1950, Dudich \& LoKsa 1969, Podlussá NY 2007, SZÉNÁsI 2012); sárgacsápú gyümölcsormányos (sárgacsápú gyümölcsfaormányos) (SÁRINGER in JERMY \& BALÁzs 1990, TóTH 1999); sárgacsápú gyümölcsormányos (HARMos et al. 2002)

\section{Laemosaccidius Smreczyński, 1972}

Magdalis (Laemosaccidius) exarata C. N. F. Brisout de Barneville, 1862 tölgyfa-magdolnaormányos (PodLUSSÁNY 2007, SzÉNÁsi et al. 2016)

\section{Magdalis Germar, 1817}

Magdalis (Magdalis) duplicata Germar, 1819 - halványcsíkú magdolnaormányos-Other names: halványcsíkú fenyőormányos (ENDRŐDI 1963, Gozmány 1979, Kasza B 1969, Podlussány 2007)

Magdalis (Magdalis) frontalis (Gyllenhal, 1827) - fényescsíkú magdolnaormányos - Other names: fényescsíkú fenyőormányos (ENDRŐDI 1963, Gozmány 1979, Kaszab 1969, Podlussány 2007)

Magdalis (Magdalis) linearis (Gyllenhal, 1827) - egyenesvállú magdolnaormányos - Other names: egyenes fenyőormányos (PoDLuSSÁNY 2007); egyenes magdolnaormányos (MERKL \& SZÉNÁSI 2018) 
Magdalis(Magdalis)memnonia(Gyllenhal,1837)-feketemagdolnaormányos (MER KL \& SZÉNÁSI 2018) - Other names: fekete fenyőormányos (ENDRŐDI 1963, Gozmány 1979, KaSZa B 1969, Podlussány 2007, SZÉNÁsi 2012)

Magdalis (Magdalis) nitida (Gyllenhal, 1827) - fényes magdolnaormányos Other names: fényes fenyőormányos (PodLussány 2007)

Magdalis (Magdalis) opaca Reitter, 1895-selymesfényü magdolnaormányosOther names: homályos fenyőormányos (PoDLussánY 2007)

Magdalis (Magdalis)phlegmatica (Herbst, 1797) - kékes magdolnaormányosOther names: kékes fenyőormányos (ENDRŐdi 1963, GozmánY 1979, PODLUSSÁNY 2007)

Magdalis (Magdalis) rufa Germar, 1824 - barnásvörös magdolnaormányos Other names: barnásvörös fenyőormányos (ENDRődi 1963, GozMÁNY 1979, Kasza b 1969, Merkl \& Vig 2009, Merkl et al.2019, Podlussány 2007)

Magdalis (Magdalis) violacea (Linnaeus, 1758) - ibolyaszínü magdolnaormányos (MERKL \& SzÉNÁsI 2018) - Other names: ibolyaszínü fenyőormányos (ENDRöDi 1963, GozmÁNY 1979, KASZAB 1969, Tóth 1999, PodLussánY 2007)

\section{Odontomagdalis Barrios, 1984}

Magdalis (Odontomagdalis) armigera (Geoffroy, 1785) - szilfamagdolnaormányos(MER KL \&VIG 2009, MER KLetal.2019, PodLuSSÁN Y 2007)Other names: szilvafaormányos (GozMÁNY 1979); szil-magdolnaormányos (Podlussány 2009, 2014, SZÉNÁsi 2012, SZÉNÁsi et al. 2016)

Magdalis (Odontomagdalis) carbonaria (Linnaeus, 1758) - nyírfa-magdolnaormányos

Magdalis (Odontomagdalis) caucasica (Tournier, 1872) - kaukázusi magdolnaormányos (PoDLUSSÁNY 2007)

\section{Panopsis K. Daniel, 1903}

Magdalis (Panopsis) flavicornis (Gyllenhal, 1836) - szőrös magdolnaormányos - Other names: szőrös tölgyormányos (PoDlussá NY 2007)

Magdalis (Panopsis) fuscicornis Desbrochers des Loges, 1870 (= quercicola Weise, 1872) - selymes magdolnaormányos - Other names: selymes tölgyormányos (PoDLUSSÁNY 2007) 


\section{Panus Schönherr, 1823}

Magdalis (Panus) barbicornis (Latreille, 1804) (= mixta Desbrochers des Loges, 1870) - almafa-magdolnaormányos (MERKL 2018, MERKL et al. 2019) Other names: almafaormányos (GozmÁNY 1979, HARmos et al. 2002, PodLUSSÁNy 2007)

\section{Porrothus Dejean, 1821}

Magdalis (Porrothus) cerasi (Linnaeus, 1758) - meggyfa-magdolnaormányos (MER KL \& SZÉNÁSi 2018, MER KL et al. 2019) - Other names: meggyfaormányos (GoZmány 1979, PodLUSSÁNy 2007, SZÉNÁsi 2012)

Molytinae Schönherr, 1823

Cryptorhynchini Schönherr, 1825

Acalles Schönherr, 1825

Acalles Schönherr, 1825

Acalles (Acalles) camelus (Fabricius, 1792) - négypúpú zártormányos (Merkl \& Vig 2009, Podlussány 2009) - Other names: négypupú ormányos (DuDICH 1950); négypupú zártormányúbogár (PODLUSSÁNY 2007)

Acalles (Acalles) echinatus (Germar, 1824) - apró zártormányos (MERKL \& VIG 2009, PodLUSSÁNY 2007, 2010,2014) - Other names: apró zártor mányúbogár (SzÉNÁsi et al. 2016)

Acalles (Acalles) fallax Boheman, 1844 (= commutatus Dieckmann, 1844) élénkfoltos zártormányos - Other names: közönséges zártormányúbogár (PoDlussány 2007); csalfa zártormányúbogár (PoDLUSSÁNY 2010, SZÉNÁsi et al. 2016)

Acalles (Acalles) papei A. Solari et F. Solari, 1905 - balkáni zártormányos (Podlussány 2007)

Acalles (Acalles) parvulus Boheman, 1837 (= turbatus Boheman, 1844) kétpúpú zártormányos - Other names: kétpupú ormányos (DUDICH 1950)

Acalles (Acalles) petryszaki Dieckmann, 1982 (GYÖrgY \& PodlussánY 2005) - sertecsomós zártormányos

Acalles (Acalles) ptinoides (Marsham, 1802) - kecses zártormányos 


\section{Acallocrates Reitter, 1913}

Acallocrates colonnellii Bahr, 2003 (STÜBEn \& Alonso-ZARAZAGA 2013) karcsútorú zártormányos - Other names: Colonnelli-ormányos (SzÉNÁsI et al. 2016); fogasnyakú ormányos (DUDICH 1950, Dudich \& LoKSA 1969); fogasnyakú zártormányosbogár (PoDLUSSÁNY 2007); fogasnyakú zártormányos (Merkl \& Vig 2009, Podlussány 2009)

Acallocrates denticollis (Germar, 1824) - duzzadttorú zártormányos

Cryptorbynchus Illiger, 1807

Cryptorhynchus Illiger, 1807

Cryptorhynchus (Cryptorhynchus) lapathi (Linnaeus, 1758) - tarka füzormányos (BALÁs \& SÁringer 1984, ENdrödi 1963, GozmÁNy 1979, KASZab 1969, Merkl \& Vig 2009, Podlussány 2007, Szalay-Marzsó in Jermy \& Balázs 1990, Tót т 1999) - Other names: füzrontó ormányos (Dudich 1950, DudicH \& Loksa 1969, GozmÁnY 1979); tarka égerormányos (GozmÁNy 1979, TótH 1999)

Echinodera Wollaston, 1863

Echinodera Wollaston, 1863

Echinodera (Echinodera) valida (Hampe, 1864) (PodLussány 2001b) zömök zártormányos

\section{Ruteria Roudier, 1954}

Echinodera (Ruteria) hypocrita (Boheman, 1837) (PoDlussány 2001b) sávos zártormányos - Other names: avarormányos (DUDICH 1950); avarlakó zártormányúbogár (PODLUSSÁNY 2007); avarlakó zártormányos (MERKL \& VIG 2009)

Echinodera (Ruteria) major (A. Solari et F. Solari, 1907) (MER KL et al. 2010) avarlakó zártormányos

Kyklioacalles Stüben, 1999

Kyklioacalles Stüben, 1999

Kyklioacalles (Kyklioacalles) suturatus (Dieckmann, 1983) (PodLussánY 2001b) - vöröses zártormányos - Other names: vöröses zártormányúbogár (PodLUSSÁNY 2007) 
Palaeoacalles Stüben, 2005

Kyklioacalles (Palaeoacalles) roboris (Curtis, 1834) - tölgy-zártormányos

Onyxacalles Stüben, 1999

Onyxacalles Stüben, 1999

Onyxacalles (Onyxacalles) croaticus (H. Brisout de Barneville, 1867) - horvát zártormányos

Onyxacalles (Onyxacalles) luigionii (A. Solari et F. Solari, 1907) - alpesi zártormányos

Gasterocercini Zherikhin, 1991

Gasterocercus Laporte et Brullé, 1828

Gasterocercus depressirostris depressirostris (Fabricius, 1792) - laposorrú ormányos (MERKL \& SZÉNÁsi 2018, Merkl \& Vig 2009, Merkl et al. 2019, PODLUSSÁNY 2007, 2009) - Other names: laposorrú ormányosbogár (HARMOS et al. 2003)

Ithyporini Lacordaire, 1865

Camptorhinus Schönherr, 1825

Camptorbinus simplex Seidlitz, 1867 (György \& Podlussány 2005) sima holttettetős-ormányos (MERKL \& VIG 2009) - Other names: mozdulatlan ormányos (PoDLUSSÁNY 2007)

Camptorbinusstatua (Rossi, 1790)-bordásholttettetős-ormányos(MER KL \& VIG 2009) - Other names: holttettetős ormányos (DUDICH 1950, PoDlussáNY 2007)

Lepyrini W. Kirby, 1837

Lepyrus Germar, 1817 2007)

Lepyrus armatus Weise, 1893 - tompafogú füzormányos (PoDlussánY

Lepyrus capucinus (Schaller, 1783) - pettyes füzormányos (BALÁs \& Sáringer 1984, Kaszab 1969, Merkl 2018, Merkl \& SzénÁsi 2018, 
Podlussány 2007, 2009, SÁRINGER in JeRMy \& BALÁzs 1990, SZÉnÁsi 2012, SZÉNÁsi etal.2016, Tó TH 1999) - Other names: kétpettyesfüzormányos(DUDICH 1950); négypettyes füzormányos, pettyes füzormányos (GozmÁNY 1979); pettyes ormányos (BALÁs \& SÁRINGER 1984); szamóca-ormányos (pettyes ormányos) (SÁRINGER in JERMY \& BALÁzs 1990); szamóca-ormányos (HARMOS et al. 2002)

Lepyrus palustris palustris (Scopoli, 1763) - kétpettyes füzormányos (ENDRődi 1963, GozmÁNy 1979, KASZAB 1969, MERKL \& SzÉnÁsi 2018, Merkl \& Vig 2009, Podlussány 2007, SzÉnÁsi 2012, SzÉnÁsi et al. 2016, TóT H 1999) - Other names: négypettyes füzormányos (DUDICH 1950, SÁRINGER in JERMY \& BALÁZS 1990)

Mecysolobini Reitter, 1913

Alcidodes Marshall, 1939

Alcidodes karelinii Boheman, 1844 (SZÉNÁsI \& VÁRI 2019) - pompás szulákormányos (SZÉNÁsI \& VÁRI 2019)

Molytini Schönherr, 1823

Adexius Schönherr, 1834

Adexius scrobipennis Gyllenhal, 1834 - gödröshátú sünormányos - Other names: havasi ormányos (GoZmáNY 1979)

$$
\begin{gathered}
\text { Hylobius Germar, } 1817 \\
\text { Callirus Dejean, 1821 } \\
\text { (= Hylobitelus Reitter, 1923) }
\end{gathered}
$$

Hylobius (Callirus) abietis (Linnaeus, 1758) (Fig. 8) - nagy fenyvesormányos (ENDRöDi 1963, MERKL \& Vig 2009, SZÉnÁsi 2012) - Other names: nagy fenyves ormányosbogár (EмICH 1899); fenyvesormányos (DUDICH 1950); nagy fenyvesbogár, nagy fenyőormányos, fenyvesormányos, fenyvesbogár (GOZMÁNY 1979); nagy fenyőormányos (ENDRŐDI 1963, KASZAB 1969, Podlussány 2007, Tóth 1999)

Hylobius (Callirus) pinastri (Gyllenhal, 1813) - kis fenyvesormányos (ENDRőDI 1963, MERKL \& VIG 2009) - Other names: kis fenyőormányos (ENDRöDi 1963, KASZAB 1969, TóTH 1999); kisebb fenyvesbogár, kis fenyőormányos (GoZMÁNY 1979) 
Hylobius(Callirus)transversovittatus(Goeze, 1777)-füzény-fenyvesormányos (Merkl \& SZÉnÁsi 2018, Merkl \& Vig 2009, Merkl et al. 2019, PodlussánY 2009) - Other names: lomberdőormányos (DUdich 1950, Gozmány 1979, SZÉNÁsI et al. 2016); barna füzényormányos (PODLUSSÁNY 2007)

Hylobius (Hylobius) Germar, 1817

Hylobius (Hylobius) excavatus (Laicharting, 1781) (= piceus DeGeer, 1775) - szurkos fenyvesormányos (MERKL \& VIG 2009) - Other names: lucfenyőormányos (ENDRŐDI 1963, KASZAB 1969, TóTH 1999); vörösfenyőormányos, lucfenyőormányos (GOZMÁNY 1979)

Leiosoma Stephens, 1829

(= Liosoma Agassiz, 1846)

Leiosoma baudii Bedel, 1884 - barnalábú barkócska

Leiosoma concinnum Boheman, 1842 - bordás barkócska

Leiosoma cribrum (Gyllenhal, 1834) (= carpathicum Brancsik, 1899) - violabarkócska (PodLussánY 2007)

Leiosoma deflexum (Panzer, 1795) - fogas barkócska (PoDlussány 2007)

Leiosoma oblongulum Boheman, 1842 - szellőrózsa-barkócska (PODLUSSÁNY 2007)

Liparus Olivier, 1807

Liparus Olivier, 1807

Liparus (Liparus) coronatus (Goeze, 1777) - kis turbolyaormányos - Other names:turbolyaormányos(DUDICH 1950, DUDICH \&LOKSA 1969, GoZMÁNY 1979, MER Kl \& Vig 2009, Podlussány 2007)

Liparus (Liparus) dirus (Herbst, 1795) - sujtár-szerecsenományos - Other names: szerecsenormányos (DUdich 1950, Dudich \& LoKSA 1969, MerkL \& Vig 2009, Podlussány 2007)

Liparus (Liparus) germanus (Linnaeus, 1758) - német acsalapuormányos (PodlussáNy 2007) - Other names: acsalapuormányos (DUDICH 1950, GoZM ÁNY 1979); kis acsalapuormányos (MERKL \& VIG 2009)

Liparus (Liparus) glabrirostris (Küster, 1849) - közönséges acsalapuormányos (Merkl \& Vig 2009, Podlussány 2007) - Other names: simaormányu Munkász (I. FRIVALDSZKY 1865); óriásormányos (GoZMÁNY 1979)

Liparus (Liparus) transsilvanicus Petri, 1895 - erdélyi acsalapuormányos (PodLUSSÁNy 2007) 


\section{Minyops Schönherr, 1823}

Minyops costalis Gyllenhal, 1834(MER KL et al.2014) - keleti bordásormányos (MERKL et al. 2014, SzÉNÁsi et al. 2016)

Minyops variolosus (Fabricius, 1775) - nyugati bordásormányos (MER KL \& SZÉNÁsi 2018) - Other names: bordás ormányos (GOZMÁNY 1979, MERKL \& Vig 2009, PodLussány 2007, 2009, SZÉNÁSI 2012)

\section{Neoplinthus Bedel, 1884}

Neoplinthus tigratus porculus (Fabricius, 1801) (= porcatus Panzer, 1798) barnás komlóormányos - Other names: komlóormányos (DUDICH 1950, GoZmány 1979, PodLussány 2007)

Plinthus Germar, 1817

Plinthomeleus Reitter, 1913

Plinthus(Plinthomeleus)squaliduselekesi Ormay, 1888(PodLuss ÁnY 2007)pannon földiormányos - Other names: Elekes-ormányos (PoDLussáNY 2007, SZÉNÁsi et al. 2016)

Plinthus (Plinthomeleus) sturmii sturmii Germar, 1818 - bordáshátú földiormányos - Other names: Sturm-ormányos (PoDlussány 2007); Sturm Téglárja (I. FRIVALDSZKY 1865); szárnyatlan ormányos (GozM ÁNY 1979)

\section{Plinthus Germar, 1817}

Plinthus (Plinthus) findelii Boheman, 1842 - fehérsávos földiormányos Other names: Findel-ormányos (PoDlussány 2007); Findely téglárja (I. FRIVALDSZKY 1865)

Plinthus (Plinthus) megerlei (Panzer, 1803) - bütyköshátú földiormányos Other names: Megerle-ormányos (PodLussány 2007)

Plinthus (Plinthus) tischeri Germar, 1824 - kárpáti földiormányos - Other names: Tischer-ormányos (PodLussánY 2007) 
Pissodini Gistel, 1848

Pissodes Germar, 1817

Pissodes Germar, 1817

Pissodes (Pissodes) castaneus (DeGeer, 1775) (= notatus Fabricius, 1787) fehérfoltos fenyőbogár (Dudich 1950, Gozmány 1979, Podlussány 2007, TóTH 1999) - Other names: kis fehérfoltos fenyőbogár, (GoZMÁNY 1979)

Pissodes (Pissodes) harcyniae (Herbst, 1795) - nagy lucfenyőbogár - Other names: lucfenyőbogár (ENDRőDI 1963); gyantás fenyőbogár, lucfenyőbogár (GoZMÁNY 1979)

Pissodes (Pissodes) piceae (Illiger, 1807) - közönséges fenyőbogár - Other names: fenyőbogár (ENDRŐDI 1963); jegenyefenyőbogár, fenyőbogár (GoZMÁNY 1979)

Pissodes (Pissodes) pini pini (Linnaeus, 1758) - öves fenyőbogár (DuDICH 1950, ENDRÖDi 1963, GozMány 1979, PodLussány 2007)

Pissodes (Pissodes) piniphilus (Herbst, 1797) - erdeifenyö-bogár (ENDRŐDI 1963, Gozm ÁNy 1979, PodLussány 2007, Tóth 1999) - Other names: kétfoltos fenyőbogár, (GoZMÁNY 1979)

Pissodes (Pissodes) scabricollis Miller, 1859 - kis lucfenyőbogár - Other names: érdestorju Szurkocs (I. FrIVALDSZKY 1865); foltos lucfenyőbogár (GOZMÁNy 1979)

Pissodes (Pissodes) validirostris (C. R. Sahlberg, 1834) - tobozevő fenyőbogár (ENDRődi 1963, GozMÁNY 1979, PODLUSSÁNY 2007)

Trachodini Gistel, 1848

Trachodes Germar, 1824

Trachodes Germar, 1824

Trachodes (Trachodes) hispidus (Linnaeus, 1758) - bozontos ormányos (Merkl \& Vig 2009, Podlussány 2007, 2010)

Typoderini Voss, 1965

Aparopion Hampe, 1861

Aparopion costatum (Fåhraeus, 1843) (Podlussány 2001b) - csipkézett bordáshátú-ormányos - Other names: csipkézett bordásormányos (SzÉNÁsI et al. 2016) 


\title{
Platypodinae Shuckard, 1840 \\ Platypodini Shuckard, 1840
}

\section{Platypus Herbst, 1794}

Platypus cylindrus (Fabricius, 1792) - közönséges hosszúlábúszú (MER KL \& VIG 2009) - Other names: hengeres törzsszú (DUDICH 1950); hosszúlábú szú (ENDRőDI 1959b, KASZAB 1969); hengeres törzsszú, hosszúlábú szú (GozMÁNY 1979, Тóth 1999)

\section{Treptoplatypus Schedl, 1972}

Treptoplatypus oxyurus (Dufour, 1843) - ékes hosszúlábúszú

\author{
Scolytinae Latreille, 1804 \\ Corthylini LeConte, 1876
}

Pityophthorus Eichhoff, 1864

Pityophthorus carniolicus Wichmann, 1910 - parányi nyurgaszú

Pityophthorus exsculptus (Ratzeburg, 1837) - lucfenyő-nyurgaszú

Pityophthorus henscheli Seitner, 1887 - kis nyurgaszú

Pityophthorus lichtensteinii (Ratzeburg, 1837) - nagy nyurgaszú

Pityophthorus micrographus (Linnaeus, 1758) - törpe nyurgaszú (MER KL \& VIG 2009) - Other names: törpe fenyőszú (DUDICH 1950); északi törpe-fenyőszú, törpe-fenyőszú (GozMÁNY 1979)

Pityophthorus pityographus pityographus (Ratzeburg, 1837) - közönséges nyurgaszú (MER KL et al. 2019)

Pityophthorus pubescens (Marsham, 1802) - barázdás nyurgaszú - Other names: barázdázott fenyőszú (KASZA B 1969)

Cryphalini Lindemann, 1876

\section{Cryphalus Erichson, 1836}

Cryphalus asperatus (Gyllenhal, 1813) (= abietis Ratzeburg, 1837) jegenyefenyőszú

Cryphalus piceae (Ratzeburg, 1837) - lucfenyőszú - Other names: jegenyefenyőszú (GoZMÁNY 1979)

Cryphalus saltuarius Weise, 1891 (PoDlussány 1999) - apró fenyőszú 
Ernoporicus Berger, 1917

Ernoporicus caucasicus (Lindemann, 1876) - kaukázusi szú

Ernoporicus fagi (Fabricius, 1798) - közönséges bükkszú (ENDRÖDI 1959) Other names: kis bükkszú (DUDICH 1950); bükkszú (ENDRŐDI 1959, GOZMÁNY 1979, Jolsvay et al. 1977, KAsza B 1969)

Ernoporus C. G. Thomson, 1859

Ernoporus tiliae (Panzer, 1793) - hamvas hársszú (DUDICH 1950, MERKL et al. 2019) - Other names: hársszú (Dudich 1950, Dudich \& LoKsA 1969, ENDRődi 1959, Gozmány 1979, Podlussány 2010)

Trypophloeus Fairmaire, 1864

Trypophloeus binodulus (Ratzeburg, 1837) - rezgőnyárszú (DUDICH 1950, GOZMÁNY 1979)

Trypophloeus granulatus (Ratzeburg, 1837) - fehérnyárszú (DuDICH 1950, Gozmány 1979, MER KL \& SzÉNÁsi 2018, MER KL et al. 2019)

Trypophloeus rybinskii rybinskii Reitter, 1895 (PodLussáNY 1998) szőröshátú szú

Crypturgini LeConte, 1876

Crypturgus Erichson, 1836

Crypturgus cinereus (Herbst, 1794) - matt fenyőszú (MER KL et al. 2019)

Crypturgus hispidulus C. G. Thomson, 1870 - szőrös fenyőszú (GozmánY 1979)

Crypturgus pusillus (Gyllenhal, 1813) - parányi fenyőszú (DUDICH 1950, GoZMÁNY 1979)

Dryocoetini Lindemann, 1877

Coccotrypes Eichhoff, 1878

Coccotrypes dactyliperda (Fabricius, 1801) (GYÖRGY \& PoDLussánY 2005) datolyaszú 


\section{Dryocoetes Eichhoff, 1864}

Dryocoetes autographus (Ratzeburg, 1837) - rozsdavörös fenyőszú (DUDICH 1950, GozMÁNy 1979)

Dryocoetes hectographus Reitter, 1913 - hegyvidéki fenyőszú - Other names: nagy égerszú (GozMÁNY 1979)

Dryocoetes villosus villosus (Fabricius, 1792) - gesztenyeszú (Dudich 1950, Gozmány 1979, Merkl \& SZÉnÁsi 2018, Merkl et al.2019, Podlusś́ny 2010)

Lymantor Løvendal, 1889

Lymantor coryli (Perris, 1855) - mogyorószú (DUDICH 1950, ENDRöDi 1959, Gozmány 1979, MERKL et al. 2019)

\section{Taphrorychus Eichhoff, 1878}

Taphrorychus bicolor (Herbst, 1794) - bóbitás bükkszú (DuDICH 1950, Dudich \& LoKsa 1969, Gozmány 1979, Jolsvay et al. 1977, MERkl et al. 2019)

Taphrorychus hirtellus Eichhoff, 1878 (= mecedanus Reitter, 1913) fénytelen szú et al. 2019)

Taphrorychus villifrons (Dufour, 1843) - mediterrán pamacsosszú (MER KL

Thamnurgus Eichhoff, 1864

Parathamnurgus Mandelshtam, Petrov et Korotyaev, 2011

Thamnurgus (Parathamnurgus) kaltenbachii Bach, 1849 - gyapjas dudvaszú (DUdich 1950, MERKL \& Vig 2009)

\section{Thamnurgus Eichhoff, 1864}

Thamnurgus (Thamnurgus) varipes Eichhoff, 1878 - kutyatejszú

\section{Xylocleptes Ferrari, 1867}

Xylocleptes bispinus (Duftschmid, 1825) - iszalagszú (ENDRődi 1959, Kaszab 1969, Merkl et al. 2019, Podlussány 2014) - Other names: kétfogú iszalagszú (DUdich 1950) 
Hylastini LeConte, 1876

Hylastes Erichson, 1836

Hylastes angustatus (Herbst, 1794) - karcsú gyökérszú (Dudich 1950, DUdich \& LoKsa 1969, GozMÁNy 1979, MER KL et al.2019, MER KL \& VIG 2009, Тóтн 1999)

Hylastes ater (Paykull, 1800) - fekete gyökérszú (Du Dich 1950, Dudich \& LoKsa 1969, GozmÁNy 1979, Tó TH 1999) - Other names: fekete fenyohháncsszú (GOZMÁNY 1979)

Hylastes attenuatus Erichson, 1836 - keskeny gyökérszú (DUdich 1950, GoZMÁNy 1979) - Other names: nyúlánk gyökérszú (GozMÁNY 1979)

Hylastes brunneus (Erichson, 1836) - barna gyökérszú

Hylastes cunicularius Erichson, 1836 - fenyő-gyökérszú (Gozmány 1979, KASZAB 1969, MERKL \& VIG 2009) - Other names: fekete lucfenyő gyökérszú (fekete gyökérszú) (GozMÁNY 1979, TóTH 1999)

Hylastes linearis Erichson, 1836 - nyúlánk gyökérszú (DUDICH 1950)

Hylastes opacus Erichson, 1836 - sötét gyökérszú (GozmáNY 1979, KASZA B 1969, MERKL et al. 2019, TóTH 1999)

Hylurgops LeConte, 1876

Hylurgops glabratus (Zetterstedt, 1828) - nagy gyökérszú - Other names: luc-gyökérszú (GoZMÁnY 1979) 1979)

Hylurgops palliatus (Gyllenhal, 1813) - rozsdavörös gyökérszú (GozMÁNY

Hylesinini Erichson, 1836

Hylastinus Bedel, 1888

Hylastinus obscurus (Marsham, 1802) - vöröshereszú (GozMÁNY 1979, Kaszab 1969, Merkl \& Vig 2009, Sáringer in Jermy \& Balázs 1990) Other names: vöröshere-gyökérszú (MAN INGER 1960, GozMÁNY 1979)

Hylesinus Fabricius, 1801

Hylesinus crenatus (Fabricius, 1787) - nagy kőrisszú (Gozmány 1979, MERKL \& Vig 2009, Tóth 1999) - Other names: kőrisfa nagy háncsszúja (GoZMÁNY 1979) 
Hylesinus toranio (Danthoine, 1788) (= oleiperda Fabricius, 1792) olajfarontó szú - Other names: szőrös olajfaszú (GozMÁnY 1979)

Hylesinus varius (Fabricius, 1775 (= fraxini Panzer, 1799) - közönséges kőrisszú (MERKL \& VIG 2009, MERKL et al. 2019) - Other names: kőrisszú (KASZAB 1969); kőrisháncs-bogár (EMICH 1899); pikkelyes kőrisszú, kőrisszú, kőrisfa kis háncsszúja, kőris háncsbogara (Gozmá NY 1979); kőrisszú (pikkelyes kőrisszú) (Тó TH 1999)

Hylesinus wachtli orni Fuchs, 1906 - csupasztorú körisszú (MER KL \& VIG 2009) - Other names: vöröseslábú kőrisszú (GOZMÁNY 1979)

Kissophagus Chapuis, 1869

Kissophagusvicinus (Comolli, 1837) (= hederaeSchmitt, 1843) - borostyánszú (ENDRődi 1959, GozMÁNy 1979, Jolsvay et al. 1977, MER KL \& VIG 2009)

\section{Pteleobius Bedel, 1888}

Pteleobiuskraatzii (Eichhoff, 1864) - tarka szilszú (DUDICH 1950, MER KL \& SZÉnÁsi 2018, MER KL \& Vig 2009, MER KL et al. 2019)

Pteleobius vittatus (Fabricius, 1792) - csíkos szilszú (MerkL \& VIG 2009, MERKL et al. 2019, PodLussánY 2014) - Other names: szilszú (ENDRöDi 1959); öves szilszú (Dudich 1950, Dudich \& LoKsa 1969)

Hylurgini Gistel, 1848

Dendroctonus Erichson, 1836

Dendroctonusmicans (Kugelann, 1794) - óriás-fenyőháncsszú(Du DICH 1950, MerKL \& Vig 2009, Tóth 1999)

\section{Hylurgus Latreille, 1806}

Hylurgus ligniperda (Fabricius, 1787) - gyökérháncsszú (GozmÁNy 1979, KASZab 1969, MerkL \& SzÉNÁsi 2018, Tóth 1999) 
Tomicus Latreille, 1802

Tomicus minor (Hartig, 1834) - kis fenyőháncsszú (Dudich 1950, Gozmány 1979, Merkl \& Vig 2009, Tóth 1999) - Other names: fenyőbélbogár (ЕмICH 1899); kis fenyőbélszú (Тó Tн 1999)

Tomicus piniperda (Linnaeus, 1758) - nagy fenyőháncsszú (DuDICH 1950, GoZM ÁNY 1979, MER KL \& VIG 2009, Tóth 1999) - Other names: fenyőháncsszú (ENDRőDI 1959); fekete fenyő-háncsszú, fenyőháncsszú, fenyőbél nagy szúja (GozmÁnY 1979); fenyőbélszú (Tóth 1999)

Hypoborini Nüsslin, 1911

Hypoborus Erichson, 1836

Hypoborus ficus Erichson, 1836 - fügeszú (ENDRöDI 1959, MER KL \& VIG 2009)

Liparthrum Wollaston, 1854

Liparthrum bartschti Mühl, 1891 - fagyöngyszú (ENDRőDI 1959)

Ipini Bedel, 1888

Ips DeGeer, 1775

Ips acuminatus (Gyllenhal, 1827) - kis betűzőszú (GozmÁNy 1979, JoLSvAY et al. 1977, KAsza B 1969) - Other names: hatfogú fenyőszú (Dudich 1950); hétfogú fenyőszú (GozMÁNY 1979, TóTH 1999)

Ips amitinus (Eichhoff, 1872) - háromszögfogú betüzőszú (MERKL \& VIG 2009) - Other names: kis betűzőszú (DUDICH 1950, GozMÁNY 1979)

Ips cembrae (Heer, 1836) - vörösfenyö-betüzőszú - Other names: sokfogú szú, barázdáltfarú szú (GOZMÁNY 1979); vörösfenyőszú (ENDRőDI 1959, TóTH 1999)

Ips duplicatus (C. R. Sahlberg, 1836) - sorpontos betűzőszú - Other names: hegyesfogú szú (GoZmánY 1979)

Ips sexdentatus (Börner, 1776) - hatfogú betüzőszú (MERKL \& VIG 2009) Other names: tucatfogú fenyőszú (Dudich 1950); tizenkétfogú szú, hatfogú szú, tucatfogú fenyőszú (GozMáNY 1979); hatfogú szú (ENDRődi 1959, TóTH 1999)

Ips typographus (Linnaeus, 1758) - közönséges betűzőszú (MERKL \& VIG 2009) - Other names: betüzőszú (EMICH 1899, DUdich 1950, Dudich \& LoKsA 1969, ENDRöDi 1959, Jolsvay et al. 1977, Tóth 1999, PodLuss ÁnY 2010) - Other names: közönséges kéregszú, betűzőszú, nyolctüskés fenyőszú (GozMÁNY 1979) 


\section{Orthotomicus Ferrari, 1867}

Orthotomicus erosus (Wollaston, 1857) - kevésfogú fogasszú - Other names: kevésfogú szú (GOZMÁNY 1979)

Orthotomicus laricis (Fabricius, 1792) - vörösfenyö-fogasszú (ЕMICH 1899, Merkl 2018, Merkl \& SzénÁsi 2018, Merkl et al. 2019) - Other names: vörösfenyőszú (ЕMICH 1899, DudicH 1950, DUdich \& LoKsA 1969, GozMÁNY 1979)

Orthotomicus longicollis (Gyllenhal, 1827) (GYÖRGY \& PODLUSSÁNY 2005) hosszúnyakú fogasszú (GozmánY 1979, MER KL \& VIG 2009)

Orthotomicus mannsfeldi (Wachtl, 1880) - nagy fogasszú

Orthotomicus proximus (Eichhoff, 1868) - erdeifenyö-fogasszú (GozMÁNY 1979, Kasza B 1969, Merke \& Vig 2009, Tóth 1999)

Orthotomicus robustus (Knotek, 1899) - vaskos fogasszú (MER KL et al. 2019)

Orthotomicus suturalis (Gyllenhal, 1827) - fekete fogasszú (GozMÁNY 1979, KASZAB 1969)

\section{Pityogenes Bedel, 1888}

Pityogenes bidentatus (Herbst, 1784) - kétfogú firkálószú - Other names: kétfogú szú, kétfogú fenyőkéregszú (GozMÁNY 1979)

Pityogenes bistridentatus (Eich hoff, 1878) - fenyő-firkálószú - Other names: horogfogú szú (GozmÁNY 1979)

Pityogenes chalcographus (Linnaeus, 1760) - hatfogú firkálószú (MERKL \& VIG 2009, MERKL et al. 2019) - Other names: hatfogú fenyőkéregszú (DUDICH 1950, Gozmány 1979); firkálószú (ENDRődi 1959, Gozmány 1979, KASZAB 1969); rézmetszőszú (GozMÁNY 1979, TóTH 1999)

Pityogenes conjunctus Reitter, 1887 - kampós firkálószú

Pityogenes quadridens (Hartig, 1834) - négy fogú firkálószú - Other names: négyfogú szú (GozMÁNY 1979, KASZA в 1969, Tó тн 1999)

Pityogenes trepanatus (Nördlinger, 1848) - ritkásfogú firkálószú (MER KL \& VIG 2009, MERKL et al. 2019) - Other names: gödröshomlokú szú (Tót H 1999)

\section{Pityokteines Fuchs, 1911}

Pityokteines curvidens (Germar, 1824) - görbefogú szú (DuDich 1950, GozmÁNy 1979, MerkL \& Vig 2009, Tóth 1999) - Other names: horogfogú fenyőszú (Dudich 1950, Gozmány 1979); jegenyefenyő szú (Merkl \& Vig 2009, То́тн 1999)

Pityokteines vorontzowi (Jacobson, 1896) - vastagfogú szú (GoZMÁNY 1979, KASZAB 1969) 
Phloeosinini Nüsslin, 1912

Phloeosinus Chapuis, 1869

Phloeosinus aubei (Perris, 1855) - borókaszú (ENDRöDi 1959, Jolsvay et al. 1977, Merkl \& Vig 2009, Merkl et al.2019, Reiderné \& Podlussány 1994, Tóтн 1999)

Phloeosinusthujae(Perris, 1855)-tujaszú(EndRöDi 1959, Mer KL \&Vig 2009, Tót H 1999) - Other names: kis tujaszú (MERKL et al. 2019)

Phloeotribini Chapuis, 1869

Phloeotribus Latreille, 1797

Phloeotribus caucasicus Reitter, 1891 (PoDlussánY 2001b) - kaukázusi kőrisszú Phloeotribus muricatus (Eggers, 1929) (PodLussány et al.2017) - apró kőrisszú Phloeotribus rhododactylus (Marsham, 1802) - seprüzanótszú

Phloeotribus scarabaeoides (Bernard, 1788) - lemezescsápú szú

Polygraphini Chapuis, 1869

Carphoborus Eichhoff, 1864

Carphoborus minimus (Fabricius, 1798) - törpe-fenyőszú (GozmÁNy 1979)

Polygraphus Erichson, 1836

Polygraphus grandiclava C. G. Thomson, 1886 - irkáló fenyőszú - Other names: nagycsápú gyümölcsszú (GozMÁNY 1979)

Polygraphuspoligraphus (Linnaeus, 1758) - kétszemű fenyőszú - Other names: firkáló fenyőszú (Dudich 1950, Gozmány 1979, Tóth 1999); kétszemű szú (GozmÁnY 1979)

Polygraphus subopacus C. G. Thomson, 1871 (Podlussány 2001b) szemölcsös fenyőszú 
Scolytini Latreille, 1804

Scolytus Geoffroy, 1762

Scolytus carpini (Ratzeburg, 1837) - gyertyán-kéregszú (Dudich 1950, GoZMÁNY 1979)

Scolytus ensifer Eichhoff, 1881 - törös szil-kéregszú

Scolytus intricatus (Ratzeburg, 1837) - tölgy-kéregszú (DuDICH 1950, Dudich \& LoKsa 1969, Gozmány 1979, Mer KL \& Szénási 2018, Merkl \& Vig 2009, MERKL et al. 2019, TóTh 1999) - Other names: tölgy háncsbogara (GozMÁNy 1979)

Scolytus kirschii kirschii Skalitzky, 1876 - ripacsoshátú kéregszú

Scolytus koenigi Schevyrew, 1890 - juhar-kéregszú - Other names: juharkéregszú (GozmánY 1979)

Scolytus laevis Chapuis, 1869 - fényeshátú kéregszú - Other names: középső szilszijács-szú (GozMÁNY 1979, Tót 1999)

Scolytus mali (Bechstein, 1805) (Fig. 9) - nagy gyümölcsfakéregszú (Dudich 1950, GozmánY 1979, SÁrINGER in JERMY \& BALÁzs 1990) - Other names: szilvakéregszú (JABLONOWsKi 1896); nagy gyümölcsfaszú (ENDRÖDI 1959); gyümölcsfa-kéregszú, szilvakéregszú, nagy kéregszú, nagy gyümölcsfaszú (GozMÁNY 1979); nagy kéregszú (BALÁs \& SÁRINGER 1984); nagy kéregszú (nagy gyümölcsfaszú, szilvakéregszú) (SÁRINGER in JERMY \& BALÁzs 1990); nagy gyümölcsfaszú (nagy kéregszú, szilvakéregszú) (TóTH 1999)

Scolytus multistriatus (Marsham, 1802) - kis szil-szijácsszú (GozMánY 1979, KASZAв 1969, Tótн 1999) - Other names: kis szilfakéregszú (Dudich 1950); vonalas gyümölcsfakéregszú, szilva kis kéregszúja, kis szilfakéregszú, csíkosszú (GozmÁnY 1979)

Scolytus pygmaeus (Fabricius, 1787) - szilfaágszú (DUDICH 1950, MERKL et al. 2019)

Scolytus ratzeburgii E. W. Janson, 1856 - nyírfa-kéregszú (DUDICH 1950, Dudich \& Loksa 1969, Gozmány 1979, Mer KL \& Vig 2009, Tóth 1999)

Scolytus rugulosus (P. W. J. Müller, 1818) - kis gyümölcsfakéregszú (DUDICH 1950, Gozmány 1979, Merkl \& Vig 2009, Merkl et al. 2019, SÁringer in JERMY \& BALÁzs 1990) - Other names: kis gyümölcsfa-Scolytus (SAJó 1893); ráncos kéregszú (JABLONOWSKI 1896); kis gyümölcsfa szú (ENDRÖDI 1959, KASZAB 1969); kis kérefszú (BALÁs \& SÁRINGer 1984); kis kéregszú (kis gyümölcsfaszú, kis gyümölcsfa Scolytus, ráncos kéregszú) (SÁ R INGER in JER MY \& BALÁzs 1990); kis gyümölcsfaszú, kis kéregszú, ráncos kéregszú, (GozMÁNY 1979); kis gyümölcsfaszú (kis kéregszú, ráncos kéregszú) (Tó TH 1999)

Scolytus scolytus (Fabricius, 1775) - nagy szilfa-kéregszú (DUDICH 1950, GOZMÁNY 1979, MER KL \& VIG 2009) - Other names: nyírháncs-bogár (ЕMICH 1899); szil háncsbogara, nagy szil-szijácsszú, szilfa nagy kéregszúja (GozMÁNY 1979); nagy szilszijács-szú (Tó тн 1999) 
Xyleborini LeConte, 1876

Anisandrus Ferrari, 1867

Anisandrus dispar (Fabricius, 1792) - kétalakú púposszú (DUDICH 1950, Gozmány 1979, Mer Kl \& Vig 2009, MER KL et al.2019, SÁRINGER in Jer My \& BALÁzs 1990, Tóth 1999) - Other names: egyenetlen szú (Divald \& WAGNER 1868); púposszú (ENDRődi 1959, GozMÁNY 1979, KASZAB 1969, BALÁs \& SÁRINGER 1984); púposszú (egyenetlen szú) (SÁr INGER in JER MY \& BALÁzs 1990, TóTH 1999)

\section{Xyleborinus Reitter, 1913}

Xyleborinus attenuatus (Blandford, 1894) (= alni Niijima, 1909) (Podlussány 2001b) - égerszú

Xyleborinus saxesenii (Ratzeburg, 1837) - vadgesztenyeszú (DUDICH 1950, Gozmány 1979, 1969, Merkl et al. 2019, Podlussány 2009, 2010, SÁRINGer in JER MY \& BALÁzs 1990, TóTH 1999) - Other names: Saxesen szúja (GozMÁNY 1979, SÁRINGER in JERMY \& BALÁZS 1990)

\section{Xyleborus Eichhoff, 1864}

Xyleborus affinis Eichhoff, 1868 - dracéna-szarvasszú

Xyleborus cryptographus (Ratzeburg, 1837) - nyár-szarvasszú (MER KL et al. 2019) - Other names: nyárszú (KASzA B 1969)

Xyleborus dryographus (Ratzeburg, 1837) - cser-szarvasszú (MERKL \& SZÉNÁsi 2018, MER KL et al.2019) - Other names: szarvas cserszú (DUDICH 1950, GOZMÁNY 1979)

Xyleborus eurygraphus (Ratzeburg, 1837) - erdeifenyö-szarvasszú - Other names: szarvas fenyőszú (TóTH 1999)

Xyleborus monographus (Fabricius, 1792) - tölgy-szarvasszú (MERKL \& SZÉNÁsi 2018, MER KL et al.2019) - Other names: szarvas tölgyszú (DUDICH 1950, Dudich \&LoKsa 1969, GozMánY 1979, MER KL \& Vig 2009, PodlussánY 2009, 2010, То́тн 1999)

Xyleborus pfeilii (Ratzeburg, 1837) - éger-szarvasszú - Other names: szarvas égerszú (Tót H 1999) 
Xylosandrus Reitter, 1913

Xylosandrus germanus (Blandford, 1894) - németszú - Other names: japán ácsszú (GoZmáNY 1979)

Xyloterini LeConte, 1876

Trypodendron Stephens, 1830

Trypodendron domesticum (Linnaeus, 1758) - nagy bükkszú (Dudich 1950, Jolsvay et al. 1977, Tótн 1999) - Other names: varratos bükkszú (JolsvaY et al. 1977, То́тн 1999)

Trypodendron lineatum (Olivier, 1795) - sávos fenyőszú (Dudich 1950, DUDICH \& LoKsa 1969, Tót H 1999)

Trypodendron signatum (Fabricius, 1792) - feketesávos szú - Other names: lombfarágó szú (KASZA B 1969, Tó TH 1999)

Acknowledgments - The authors' thanks are due to Dr George Hangay (Sydney, Australia) for compiling the text of an earlier version of the paper; to Aranka Grabant (Hungarian Natural History Museum, Budapest) for her enormous help in data input; and to everyone for participating in the increase of the checklist with their collections and publications.

\section{MAGYARORSZÁG ORMÁNYOSALKATÚINAK FAJLISTÁJA (COLEOPTERA: CURCULIONOIDEA)}

Az utolsó teljes fajlista Magyarország ormányosalkatú bogarairól 1996ban jelent meg (Podlussány 1996). Azóta számos kisebb-nagyobb közlemény látott napvilágot az ország faunájában először előkerült és a tudomány számára újonnan leírt fajokról. Egy teljes, a hazai ormányosalkatú faunát tárgyaló fajlista fontosságát a közelmúltban megjelent (LöBL \& SMETANA 2011, 2013), majd hamarosan javított változatban is kiadott palearktikus bogárkatalógusban (Alonso-ZARAZAGA et al. 2017) található téves információk nagy száma is indokolja. Az örvendetesen erősödő hazai nem hivatásos kutatói közösség munkája és a „citizen science” (civil kutatás, közösségi tudomány) is igényel egységes nevezéktant használó, napjaink ismeretanyagát tartalmazó, könnyen áttekinthető és könnyen hozzáférhető katalógust. 
Munkánk egyik fö célja az újonnan előkerült fajok felvétele mellett a különböző okok miatt tévesen jelzett (és ezért időről-időre idézett) fajok törlése katalógusunkból. A 97 faj törlésének fő okai a következők.

1. Téves határozások.

2. Fajok a Magyar Királyság területéről, mely jóval nagyobb volt, mint az 1920 -as trianoni egyezmény utáni ország. Sok faj olyan helyeken fordul elö, melyek ma a környező országokhoz tartoznak, de a jelenlegi határokon belül ismeretlen (többségük a Kárpátok nagyobb magasságaiban vagy délebbi területeken - például a Bánságban vagy a Vajdaságban - él).

3. Fajok, melyek előfordulásaként csak országot vagy nehezen definiálható régiót említenek (pl. „Hungaria” vagy „Hungaria meridionalis”); bizonyítópéldányaik, ha vannak egyáltalán, nélkülözik a pontos lelőhelyet.

4. A bizonyítópéldányok hiánya.

5. Bizonyítópéldányok léteznek, de eredetük megbízhatatlan vagy irreális. Ilyen pl. a néhai Lichtneckert Ferenc gyüjteményének számos példánya "Siófok" lelőhellyel. Lichneckert Siófokon élt és gyüjtött, de más országok bogarait is gyüjtötte vagy kapta; halála után figyelmetlenül az összes cédulázatlan példányát „Siófok” feliratú cédulával látták el, független attól, hogy azok honnan származtak.

6. Taxonómiai revíziók. Egyes genuszok vagy fajcsoportok revíziója után kiderült, hogy bizonyos fajok, melyekről azt hittük, hogy élnek Magyarországon, valójában hiányoznak az országból; a példányok más fajhoz tartoznak. Példa ilyen fajpárokra az Aphytobius sphaerion vs. veronicae (KRÁt KÝ 2015), a Minyops carinatus vs. variolosus (OSELLA \& BELLÒ 2010) vagy a Mogulones sublineellus vs. albolineatus (KRÁT KÝ \& SZYPU£A 2018).

Két faj, az Apsis hungarica (Formánek, 1925) és a Corimalia maculaticeps (Pic, 1929) önálló faji rangja kérdéses, azonban ezeket példányok hiányában nem tudtuk vizsgálni.

A listában 5 család 329 genuszának 1224 faja szerepel. A fajlista nevezéktana Alonso-ZaraZAGA et al. (2017) munkáját követi. 


\section{REFERENCES}

Alonso-Zarazaga M. A., Barrios H., Borovec R., Bouchard P., Caldara R., Colonnelli E., Gülte kin L., Hlaváč P., Korotyaev B., Lyal C. H. C., Machado A., Meregalli M., Pierotti H., Ren L., Sánchez-Ruiz M., Sforzi A., Silfyerberg H., Skuhrovec J., Trúzina M., Velázguez de Castro A. J. \& Yunakov N. N. 2017: Cooperative Catalogue of Palaearctic Coleoptera Curculionoidea. Monografías electrónicas SEA 8. - Sociedad Entomológica Aragonesa S. E. A., Zaragoza, 729 pp.

BALÁs G. 1963: Kertészeti növények állati kártevői. - Mezőgazdasági Kiadó, Budapest, 446 pp.

BALÁs G. 1966: Kertészeti növények állati kártevői. - Mezőgazdasági Könyvkiadó, Budapest, $527 \mathrm{pp}$.

BALÁs G. \& SÁRINGER Gy. 1984: Kertészeti kártevők. - Akadémiai Kiadó, Budapest, 1071 pp.

Bezsilla L., ENDRődi S. \& KACsó A. 1953: Magyarországon előforduló fontosabb kártevő állatok. - A növényvédelem időszerü kérdései 2: 29-44.

BiAŁooki P. Z. 2007: On new taxa of Entiminae (Coleoptera: Curculionidae) from Turkey and South-Eastern Europe. - Annals of the Upper Silesian Museum (Entomology) 14-15: 135-193.

Bognár S. 1952: Adatok a kendermagbogár (Peritelus familiaris Boh.) biológiájához. - Annales Instituti Protectionis Plantarum Hungarici 5: 310-311.

Caldara R. 2013: Curculionidae: Curculioninae, Bagoinae. - In: Löbl I. \& Smetana A. (eds): Catalogue of Palaearctic Coleoptera. Volume 8. Curculionoidea II. Brill, Leiden, pp. 117-176.

Dieckmann L. 1973: Die westpaläarktischen Thamiocolus-Arten (Coleoptera: Curculionidae) Beiträge zur Entomologie 23(5-8): 245-273.

Dieckmann L. \& Behne L. 1994:90. Superfamilie: Curculionoidea;93. Familie: Curculionidae. In: Lohse G. A. \& Lucht W. (eds): Die Käfer Mitteleuropas. 3. Supplementband mit Katalogteil. Goecke \& Evers, Krefeld, pp. 246-258.

Divald A. \& WagNeR K. 1868: Magyar-német és német-magyar erdészeti müszótár. - Nyomtatott Kocsi Sándornál, Pest, 68 pp.

Dudich E. 1950: 14. rend: Bogarak - Coleoptera. - In: Móczár L. (ed.): Állathatározó I. kötet. Közoktatásügyi Kiadóvállalat, Budapest, pp. 124-331.

Dudich E. \& LoKsA I. 1969: Állatrendszertan. - Tankönyvkiadó, Budapest, 708 pp.

ЕмICH G. 1899: A mezö- és kertgazdaságra káros rovarok. A gazdasági rovartan kézikönyve. I. rész. Függelékkel ellátta Jablonowski József. - Budapest, Pallas, 311 pp.

ENDRŐdi S. 1958: Eszelények - Attelabidae. - In: Magyarország Állatvilága (Fauna Hungariae), $X, 2$. Akadémiai Kiadó, Budapest, $34 \mathrm{pp}$.

ENDRÖDi S. 1959: Szúbogarak - Scolytidae. - In: Magyarország Állatvilága (Fauna Hungariae), X, 9. Akademiai Kiadó, Budapest, 96 pp.

ENDRŐDI S. 1960: Ormányosbogarak II. - Curculionidae II. - In: Magyarország Állatvilága (Fauna Hungariae), X, 5. Akadémiai Kiadó, Budapest, 126 pp.

ENDRődI S. 1961a: Ormányosalkatúak - Rhynchophora. - In: Magyarország Állatvilága (Fauna Hungariae), $X, 1$. Akadémiai Kiadó, Budapest, 24 pp.

ENDRődi S. 1961b: Ormányosbogarak I. - Curculionidae I. - In: Magyarország Állatvilága (Fauna Hungariae), X, 4. Akadémiai Kiadó, Budapest, 77 pp. 
ENDRÖDI S. 1963: Ormányosbogarak III. - Curculionidae III. - In: Magyarország Állatvilága (Fauna Hungariae), X, 6. Akadémiai Kiadó, Budapest, 104 pp.

ENDRődi S. 1968: Ormányosbogarak IV. - Curculionidae IV. - In: Magyarország Állatvilága (Fauna Hungariae), X, 7. Akadémiai Kiadó, Budapest, 129 pp.

ENdRődi S. 1971: Ormányosbogarak V. - Curculionidae V. - In: Magyarország Állatvilága (Fauna Hungariae), X, 8. Akadémiai Kiadó, Budapest, 167 pp.

EnTz F. 1859: A mogyorófáról. - Kertészeti Füzetek 14: 65-90.

FöldI J. 1801: Természeti história. A Linnaeus systemája szerént. 1. tsomó. Az állatok országa. Wéber Simon Péter, Pozsony, 428 pp.

FrivaldSZKY I. 1865: Jellemző adatok Magyarország faunájához. - A Magyar Tudományos Akadémia Évkönyvei 11(4): 1-274 + XIII.

GozMÁNY L. 1979: Vocabularium nominum animalium Europae septem linguis redactum. Európa állatvilága. Hétnyelvü névszótár. I. - Akadémiai Kiadó, Budapest, 1172 pp.

GYörfFY J. 1956: Cickányormányosok - Apionidae. - In: Magyarország Állatvilága (Fauna Hungariae), $X$, 3. Akadémiai Kiadó, Budapest, 56 pp.

GYöRFI J. 1957: Erdészeti rovartan. - Akadémiai Kiadó, Budapest, 670 pp.

GyöRgY Z. 2006: Cheklist of Hungarian Anthribidae and Urodontidae (Coleoptera). - Folia entomologica hungarica 67: 63-67.

György Z. \& Podlussány A. 2005: Notes on Curculionoidea of Hungary (Coleoptera: Anthribidae, Erirhinidae, Curculionidae, Scolytidae). - Folia entomologica hungarica 66: $57-62$.

Harmos K., Lantos I. \& Joó M. 2003: Adatok védett rovarfajok elterjedéséhez Nógrád megyében. - A Puszta 18 [2001]: 6-27.

Harmos K., Lantos I. \& Oszonics I. 2002: Adatok a Körös-Maros N. P. Igazgatóság illetékességi területének rovarfaunájához. - A Puszta 17 [2000]: 139-169.

JA BLONOWSKI J. 1896: A kéregrontó szuhokról. - A Kert 2: 666-669.

JabLONOWSKI J. 1900: Rothadó gyümölcs és férges gyümőlcs. - Természettudományi Közlöny 32: $19-25$.

Jablonowski J. 1906: A czukorrépa állati ellenségei. - Magyar Czukorgyárosok Országos Egyesületének kiadása, Budapest, 288 pp.

JA BLONOWSKI J. 1912: A gyümölcsfák s a szölő kártevő rovarai. 3. kiadás. - Pallas Részvény társaság Könyvsajtója, Budapest, 182 pp.

JA BLONOWSK I J. 1914: Elözetes tájékoztató a gubacslakó káposztabarkó (Ceutorrhynchus sulcicollis Gyll., helyesebben C. pleurostigma MSH.) ellen való védekezésről. - A Magyar Királyi Földművelésügyi Minister Kiadványa, Budapest, $16 \mathrm{pp}$.

Jenser G. 1969: Üzemi gyümölcsösök növényvédelme. Alma- és csonthéjas termésüek. Mezőgazdasági Kiadó, Budapest, 273 pp.

JERMY T. \& BALÁzs K. (eds) 1990: A növényvédelmi állattan kézikönyve 3/B. - Akadémiai Kiadó, Budapest, pp. 329-673.

Jolsvay A., Steinmann H. \& Szily E. 1977: A magyar állatvilág szótára. - Natura, Budapest, 357 pp.

Kadocsa Gy. 1923: Konyhakerti növényeink állati ellenségei. - Légrádi Testvérek Kiadása, Budapest, 122 pp. 
KA Docsa Gy. 1929: Gazdasági állattan. A hasznos és kártevő állatok ismertetése. - Pátria Irodalmi Vállalat és Nyomdai Rt., Budapest, 162 pp.

Kadocsa Gy. 1942: Gazdasági állattan. A hasznos és kártevó állatok ismertetése. Tanuló és gyakorló gazdák és kertészek számára. Második, bővitett kiadás. - Pátria Irodalmi Vállalat és Nyomdai Rt., Budapest, 242 pp.

Kasza в Z. 1969: Bogarak - Coleoptera. - In: MóczÁr L. (ed.): Állathatározó I. Tankönyvkiadó, Budapest, pp. 361-639.

KovÁcs S. 1962: Új termesztési eljárással a szamócagyökérormányos kártétele ellen. - Kertészet és Szőlészet 11(12): 20-21.

KRÁtKÝ J. 2015: Aphytobius veronicae (Frivaldszky, 1884) (Coleoptera, Curculionidae, Ceutorhynchinae, Hypurini) - species status revised. - Snudebiller 16(245): 1-8.

KRÁtKÝ J. \& Podlussány A. 2008: New weevil species in the fauna of Hungary (Coleoptera: Curculionoidea). - Folia entomologica hungarica 69: 185-188.

KRÁt KÝ J. \& SZYPUŁA J. 2018: On the validity of Mogulones albolineatus (J. Frivaldszky, 1878) (Coleoptera: Curculionidae). - Annals of the Upper Silesian Museum in Bytom, Entomology 26: 1-13. https://doi.org/10.5281/zenodo.1250759

Löbl I. \& Smetana A. (eds) 2011: Catalogue of Palaearctic Coleoptera. Volume 7. Curculionoidea I. - Brill, Leiden, 373 pp. https://doi.org/10.1163/9789004260931_012

Löbl I. \& Smetana A. (eds) 2013: Catalogue of Palaearctic Coleoptera. Volume 8. Curculionoidea II. - Brill, Leiden, 700 pp. https://doi.org/10.1163/9789004259164_010

MAN Ninger G. A. 1960: Szántóföldi növények állati kártevői. - Mezőgazdasági Kiadó, Budapest, $374 \mathrm{pp}$.

MARTINOVICH V. 1958: Megfigyelések magkártevő rovarokról. - Folia entomologica hungarica 11: $508-511$.

MerkL O. 2018: Bogarak az Ócsai Gyakorlótérről (Coleoptera). (Beetles (Coleoptera) from the Ócsa Military Training Area.) - In: Korda M. (ed.): Természetvédelem és kutatás a Turjánvidék északi részén. Tanulmánygyüjtemény. (Nature conservation and research in Northern Turján Region. Collected studies.) Rosalia (A Duna-Ipoly Nemzeti Park Igazgatóság tanulmánykötetei, 10.). Duna-Ipoly Nemzeti Park Igazgatóság, Budapest, pp. 639-664.

Merkl O., Hegyessy G., Molnár M., Németh T., Szalóki D. \& Szénási V. 2012: Seven new beetle species in the Hungarian fauna (Coleoptera). - Folia entomologica bungarica 73: 29-33.

Merkl O., Ködöвöcz V., Deli T. \& Danyik T. 2014: Bogárfaunisztikai adatok a DélTiszántúlról (Coleoptera). (Faunistic data to the beetles from the south-eastern Great Hungarian Plain (Coleoptera).) - Crisicum 8: 99-152.

Merkl O., Németh T., György Z., Podlussány A., Szelenczey B. \& Vig K. 2010: Further new beetle species in the Hungarian fauna (Coleoptera). - Folia entomologica bungarica 71: 23-29.

Merkl O., Szalóki D., Kutasi Cs., Mészáros Á., Podlussány A. \& Tallósi B. 2019: Biodiverzitás a Soroksári Botanikus Kertben - Bogarak. (Biodiversity in the Soroksár Botanical Garden - Beetles.) - Magyar Biodiverzitás-kutató Társaság \& SZIE Kertészettudományi Kar, Soroksári Botanikus Kert, Budapest, 179 pp. 
MerkL O. \& SzÉNÁsi V. 2018: A Turjánvidék Natura 2000 terület déli részének bogárfaunája (Coleoptera). (The beetle (Coleoptera) fauna of the southern part of the Turjánvidék Natura 2000 site.) - In: Korda M. (ed.): Természetvédelem és kutatás a Turjánvidék északi részén. Tanulmánygyüjtemény. (Nature conservation and research in Northern Turján Region. Collected studies.) Rosalia (A Duna-Ipoly Nemzeti Park Igazgatóság tanulmánykötetei, 10.). Duna-Ipoly Nemzeti Park Igazgatóság, Budapest, pp. 509-638.

MERKL O. \& Tusnádi Cs. K. 1985: Ritka ormányosbogár: az Otiorhynchus sulcatus Fabr. kártétele Magyarországon (Coleoptera: Curculionidae). (Damage of a rare weevil, Otiorhynchus sulcatus Fabr. in Hungary (Coleoptera: Curculionidae).) - Folia entomologica hungarica 46(1): 269-270.

MERKL O. \& VIG K. 2009: Bogarak a pannon régióban. - Vas Megyei Múzeumok Igazgatósága,

B. K. L. Kiadó, Magyar Természettudományi Múzeum, Szombathely, 494 pp.

NYÁRY F. 1877: A mezö- és kert-gazdaságra káros rovarok ismertetése és azok irtásmódja. - Franklin Társulat, Magy. Irod. Intézet és Könyvnyomda, Budapest, 112 pp.

Osella G. \& Bellò C. 2010: Revisione di Minyops Schoenherr, 1823 e Paraminyops nov. gen. (Coleoptera, Curculionidae, Molytinae). - Memorie del Museo Civico di Storia Naturale di Verona, 2. Serie, Sezione Scienze della Vita 19: 1-133.

Oszonics I. 1997: Érdekességek Túrkeve környékének ormányosbogarairól. [Interesting finds about Curculionoidea of the environs of Túrkeve, Hungary.] - A Puszta 14: 112-121.

PÁsztoR I. 1901: Az almavirág ormányos és a körterügyfúró bogár életmódja és irtása. Kisérletügyi Közlemények 4: 244-273.

PÉTERF F. 1958: Mezögazdasági rovarhatározó. - Mezőgazdasági és Erdészeti Áll. Könyvkiadó, Bukarest, $459 \mathrm{pp}$.

Podlussány A. 1984: A Bakony hegység áleszelény és eszelény faunája (Coleoptera: Rhinomaceridae, Attelabidae). (Rhinomaceridae and Attelabidae fauna of the Bakony Mountains.) - Folia musei historico-naturalis bakonyiensis 3: 57-70.

PODLUSSÁNY A. 1996: Magyarország ormányosalkatú bogarainak fajlistája (Coleoptera: Curculionoidea).(A check-list of the superfamily Curculionoidea (Coleoptera) of Hungary.) Folia entomologica hungarica 57: 197-225.

Podlussány A. 1998: A Duna-Dráva Nemzeti Park Dráva-mente Curculionoidea faunájának alapvetése (Coleoptera: Curculionoidea). (Grundlegung der Curculionoidae [sic] Fauna der Drau-Gegend im Donau-Drau Nationalpark.) - Folia entomologica hungarica 59: 271-315.

Podlussány A. 1999: Curculionoidea (Coleoptera) of the Aggtelek National Park. In: Mahunka S. (ed.): The Fauna of the Aggtelek National Park. Hungarian Natural History Museum, Budapest, pp. 291-371.

Podlussány A. 2001a: Somogy megye ormányosalkatú bogarainak katalógusa (Coleoptera: Curculionoidea). (Catalogue of the weevil fauna of Somogy county (Coleoptera: Curculionoidea.) - Natura Somogyiensis 1: 237-252.

Podlussány A. 2001b: Új ormányosalkatú bogárfajok Magyarország faunájában (Coleoptera: Curculionoidea). (Curculionoid beetle species new for the fauna of Hungary (Coleoptera: Curculionoidea).) - Folia entomologica hungarica 62: 372-378. 
Podlussány A. 2007: A Bakony ormányosbogár-faunája (Coleoptera: Brachyceridae, Curculionidae). A Bakony természettudományi kutatásának eredményei 30. (The weevil fauna of the Bakony (Coleoptera: Brachyceridae, Curculionidae. Resultationes investigations rerum naturalium montium Bakony XXX.) - Bakonyi Természettudományi Múzeum, Zirc, $224 \mathrm{pp}$.

Podlussány A. 2007: A Bakony ormányosbogár-faunája (Coleoptera: Brachyceridae, Curculionidae). A Bakony természettudományi kutatásának eredményei 30. (The weevil fauna of the Bakony (Coleoptera: Brachyceridae, Curculionidae. Resultationes investigations rerum naturalium montium Bakony XXX.) - Bakonyi Természettudományi Múzeum, Zirc, $224 \mathrm{pp}$.

PodlussánY A. 2009: A gyűrüfüi Biodiverzitás Napokon gyüjtött ormányosalkatú bogarak (Coleoptera: Curculionoidea). (A faunistical survey on Curculionoidea (Coleoptera) at Gyürüfü environs on the Biodiversity Days.) - Natura Somogyiensis 13: 135-146.

Podlussány A. 2010: A porvai Biodiverzitás napon gyüjtött ormányosalkatú bogarak (Coleoptera: Curculionoidea) (Checklist of (Coleoptera: Curculionoidea) collected on the occasion of the International Biodiversity Days 2010 (Porva, Hungary).) - Folia musei bistorico-naturalis bakonyiensis 27: 105-111.

Podlussány A., Hegyessy G. \& Kutasi Cs. 2017: Four new weevil species in the fauna of Hungary (Coleoptera, Curculionidae). - Folia entomologica hungarica 78: 83-86. https://doi.org/10.17112/FoliaEntHung.2017.78.83

Podlussány A. \& KUTAsi Cs. 2011: Új ormányosalkatú-bogárfajok a Bakonyban (Coleoptera, Curculionoidea). (New Curculionoidea beetles from the Bakony Mountains (West Hungary).) - Folia musei historico-naturalis bakonyiensis 28: 197-200.

Podlussány A., Kutasi Cs., Muskovits J. \& Rozner I. 2014: Adatok Simontornya és környékének bogárfaunájához (Coleoptera). - In: Szita É., FeTYKó K., Kovács T. \& Horváth A. (eds): Simontornya izeltlábúi. In memoriam Pillich Ferenc. Magyar Biodiverzitás-kutató Társaság, Budapest, pp. 87-106.

Podlussány A., Szita É., Lupták R., SZÉnÁsi V. \& Kiss B. 2016: Four weevil species new to the fauna of Hungary from motorway rest areas (Coleoptera: Curculionidae). - Folia entomologica hungarica 75: 73-78. https://doi.org/10.17112/FoliaEntHung.2017.78.83

REICH ART G. 1953: Erdei növények kártevői és betegségei. - In: UBRIZsY G. (ed.): A növényvédelem gyakorlati kézikönyve. Mezőgazdasági Kiadó, Budapest, pp. 641-782.

Reichart G. 1957: Megfigyelések a borsóormányos (Aoromius quinquepunctatus L.) magyarországi életmódjához. - Annales Instituti Protectionis Plantarum Hungarici 7: 313-337.

Reiderné Saly K. \& Podlussány A. 1994: A borókaszú [Phloeosinus aubei (Perris, 1855)] terjedése, gazdanövényköre és életmódja. - Növényvédelem 30(1): 23-24.

SAJó K. 1893: A gyümölcsfákban élő szúféle bogarak. - Gyümölcskertész 3: 98, 109, 153.

SAJó K. 1894: A gyümölcsfák ormányos bogarai. - Gyümölcskertész 4: 29-31.

SLÁmA P. 2015: Notes on the distribution of Curculio vicetinus (Coleoptera: Curculionidae: Curculioninae). - Klapalekiana 51(1-2): 61-70. 
Stüben P.E. \& Alonso-Zarazaga M. A. 2013: Cryptorhynchinae. - In: Löbl I. \& Smetana A. (eds): Catalogue of Palaearctic Coleoptera. Volume 8. Curculionoidea II. Brill, Leiden, pp. 229-245.

SURÁNYi P. 1946: A gyümölcs állati kártevői. - In: MocsáRY M. (ed.): A gyümölcstermesztés kézikönyve. 3. kiadás. Budapest, Pátria Irodalmi Vállalat és Nyomdai Rt., pp. 704-739.

SzÉNÁsı V. 2012: Adatok a Tápió-vidék ormányosalkatú faunájához (Coleoptera, Curculionidea). (Data on the weevil fauna (Coleoptera:Curculionidae) of Tápió region.) - In: VIDRA T. (ed.): Természetvédelem és kutatás a Tápió-vidéken. (Nature conservation and research in the Tápió Region.) Rosalia (A Duna-Ipoly Nemzeti Park Igazgatóság tanulmánykötetei, 7.) Duna-Ipoly Nemzeti Park Igazgatóság, Budapest, pp. 311-343.

SZÉNÁSI V. 2014: New and rare weevils in Hungary: distributional records and notes (Coleoptera: Curculionoidea). - Folia entomologica hungarica 75: 79-90. https://doi.org/10.17112/FoliaEntHung.2014.75.79

SZÉNÁsI V. 2016: Two new weevil species in Hungary (Coleoptera, Curculionidae: Entiminae). Folia entomologica hungarica 77: 53-55. https://doi.org/10.17112/FoliaEntHung.2016.77.53

SZÉNÁSI V. 2018: New weevils from Hungary and Slovakia (Coleoptera: Curculionidae). - Folia entomologica hungarica 79: 77-80. https://doi.org/10.17112/FoliaEntHung.2018.79.77

SZÉNÁSI V., DANYIK T. \& Deli T. 2016: Adatok a Körös-Maros köze és környéke ormányosalkatúfaunájához (Coleoptera: Curculionoidea). (Data to the weevils-fauna of the Körös-Maros region (Coleoptera: Curculionoidea).) - Crisicum 9: 171-199.

Szénási V., Podlussány A. \& Hegyessy G. 2019: New and rare weevil beetles in Hungary (Coleoptera: Curculionoidea). - Folia entomologica hungarica 80: 77-82. https://doi.org/10.17112/FoliaEntHung.2019.80.77

SZÉNÁsi V. \& VÁRI G. 2019: Alcidodes karelinii, new to Hungary (Coleoptera: Curculionidae: Molytinae):. - Folia entomologica hungarica 80: 83-87. https://doi.org/10.17112/FoliaEntHung.2019.80.88

TALlósı B. 2019: Vörös pálmaormányos előkerülése a Közép-Tisza-vidéken. - Rovarász Híradó 95: 7. Online:

https://www.rovartani.hu/wp-content/uploads/2017/11/RH95-honlapra-2019_08.pdf

Toševski I., Caldara R., Jović J., Hernández-Vera G., Baviera C., Gassmann A. \& EMERSON B. C. 2011: Morphological, molecular and biological evidence reveal two cryptic species in Mecinus janthinus Germar (Coleoptera, Curculionidae, a sucessful biological control agent of Dalmatian toadflax, Linaria dalmatica (Lamiales, Plantaginaceae). Systematic Entomology 36: 741-753. https://doi.org/10.1111/j.1365-3113.2011.00593.x

Toševski I., Caldara R., Jović J., Hernández-Vera G., Baviera C., Gassmann A. \& EMERSON B. C. 2013: Revision of Mecinus heydenii species complex (Curculionidae): integrative taxonomy reveals multiple species exhibiting host specialization - Zoologica Scripta 43(1): 34-51. https://doi.org/10.1111/zsc.12037

Toševski I., Caldara R., Jović J., Hernández-Vera G., Baviera C., Gassmann A. \& EMERSON B. C. 2015: Host-associated genetic divergence and taxonomy in the Rhinusa pilosa Gyllenhal species complex: an integrative approach. - Systematic Entomology 40(1): 268-287. https://doi.org/10.1111/syen.12109 
TóTH Gy. 1962: A szamócaültetvényeket pusztító gyökérormányos bogarak (Otiorrhynchus ovatus L. és Otiorrhynchus rugosostriatus Goeze (Coleopt. Curculionidae) életmódja, kártétele és a védekezés lehetőségei. - A Kertészeti és Szőlészeti Föiskola Évkönyve 26: 99-120.

Tó тн J. 1999: Erdészeti rovartan. - Agroinform Kiadó, Budapest, 480 pp.

Ubrizsy G. \& ReIChart G. 1958: Termesztett növényeink védelme. - Mezőgazdasági Kiadó, Budapest, 448 pp.

Wanat M., Podlussány A. \& Schön K. 2012: Perapion connexum (Schilsky, 1902) (Coleoptera, Apionidae) in Central Europe, a case of plant expansion chase. - ZooKeys 174: 49-61. https://doi.org/10.3897/zookeys.174.2526

YUnakov N. N. 2006: Contribution to a revision of the weevil genus Brachysomus Schoenh. (Coleoptera: Curculionidae: Entiminae). Description of new taxa. - Proceedings of the Russian Entomological Society 77: 294-329.

Yuna kov N. N. 2013: Entiminae: Alophini, Polydrusini, Psallidiini, Tropiphorini. - In: LöBL I. \& Smetana A. (eds): Catalogue of Palaearctic Coleoptera. Volume 8. Curculionoidea II. Brill, Leiden, pp. 251-254, 364-375, 375-376, 422-423. 